Article

\title{
First Study on Marine Heterobranchia (Gastropoda, Mollusca) in Bangka Archipelago, North Sulawesi, Indonesia
}

\author{
Adelfia Papu ${ }^{1,2, *(\mathbb{D}, \text { Nani Undap }}{ }^{1}{ }^{\mathbb{D}}$, Nancy Armas Martinez ${ }^{1}$, Marco R. Segre ${ }^{3}$, \\ Ivan Galton Datang ${ }^{3}$, Rendy Robert Kuada ${ }^{3}$, Marco Perin ${ }^{3}$, Nathalie Yonow ${ }^{4}$ and \\ Heike Wägele ${ }^{1}$ (D) \\ 1 Centre of Molecular Biodiversity, Zoological Research Museum Alexander Koenig, 53113 Bonn, Germany \\ 2 Faculty of Mathematics and Natural Sciences, Sam Ratulangi University, Manado 95115, Indonesia \\ 3 Coral Eye, Bangka Island 95375, North Sulawesi, Indonesia \\ 4 Department of Biosciences, Swansea University, Singleton Park, Swansea SA2 8PP, Wales, UK \\ * Correspondence: A.Papu@leibniz-zfmk.de
}

Received: 13 January 2020; Accepted: 25 January 2020; Published: 28 January 2020

\begin{abstract}
As ephemeral, benthic, secondary consumers usually associated with sessile coral reef organisms, marine heterobranchs are good indicators of the health of marine tropical habitats. Thus, marine Heterobranchia have recently become a major target for monitoring programs. For this work, an extensive survey was made in Bangka Archipelago, the first of its kind in this area. Bangka Archipelago is composed of small islands and the adjacent coastline of North Sulawesi. The substrate is dominated by biogenic reefs and volcanic rocks, thus forming highly diverse habitats. In total, 149 species were collected and/or photo-documented in September 2017 and September 2018, which represents the dry season. They can be assigned to the Cephalaspidea, Aplysiida, Pleurobranchida, Nudibranchia, and Eupulmonata. Thirty-three species are new to science, highlighting the lack of knowledge about the group and especially about this region. Our data provide a baseline for future monitoring surveys, as the anthropogenic pressures on Bangka Archipelago are increasing due to enhanced touristic activities, but also due to large scale environmental changes caused by previous mining operation activities.
\end{abstract}

Keywords: biodiversity; marine Heterobranchia; Bangka Island; North Sulawesi; monitoring

\section{Introduction}

Indonesia is one of the mega-diverse countries; however, surveys covering marine invertebrates in particular are very rare, these documents are often not open-access, and they are frequently in the Indonesian language [1]. The largest open-access study on Indonesian marine life is the Rumphius Biohistorical Expedition to Ambon in 1990, which has been published as a series of reports on numerous marine taxa in Zoologische Mededelingen, e.g., Naturalis Biodiversity Center [2]. This series also comprised studies on marine Heterobranchia, increasing our knowledge on this particular group considerably in an Indonesian region [3-6].

Marine heterobranchs are interesting in many ways. Many of them have reduced or lost their shells and developed alternative defense or antifouling systems, by taking up natural compounds from their food, or by de novo synthesis. These compounds are of interest as potential drug leads for medical application [7-10]. The loss of the shell allows sea slugs to develop stunning body shapes and coloration, and they are therefore well-known to snorkelers, divers, and underwater photographers [11]. The diversity of heterobranchs serves as an indication of the diversity of other metazoan life forms such 
as sponges, cnidarians, ascidians and bryozoans, as they provide nutrition with high food specificity on the species level. These filter feeders or consumers on lower levels are highly affected by changes in the environment, thus also affecting sea slug communities (e.g., [12-14]).

Burghardt et al. [15] recorded molluscan diversity for the first time in North Sulawesi (Bunaken National Park, BNP), including heterobranch taxa. More recently, several publications on heterobranch diversity have been published from North Sulawesi, including Bunaken National Park, Lembeh Strait and Sangihe Island [1,16-18], which renders this area as one of the best investigated places in Indonesia with regards to this taxon. Our study in Bangka Archipelago increases our knowledge on the biodiversity of heterobranchs in North Sulawesi in an area that has never been documented before.

Bangka Archipelago comprises small islands located in the northeast of North Sulawesi, thus lying in between the recently well-studied area of Bunaken National Park, and the famous Lembeh Strait, whereas Sangihe Island lies approximately $200 \mathrm{~km}$ to the north. All studies have shown that these areas differ in heterobranch composition due to the differing habitat compositions [16-18]. Bangka Island exhibits biogenic reefs, volcanic rocks, and a few areas with mangroves and sea grass meadows. The mainland facing Bangka Island is of volcanic origin with smaller fringing reefs. The central area of the archipelago is shallow, while the outer slopes drop down to more than $1000 \mathrm{~m}$ depth. The islands are covered by lush vegetation and are framed by fringing reefs, alternating with few mangroves and volcanic cliffs. The islands are home to some villages and a few resorts, of which the oldest one was built in 1987. Bangka, the largest island of this archipelago (approximately $48 \mathrm{~km}^{2}$ ), has a resident population of nearly 2500 inhabitants and only six resorts [19]. Therefore, this island is less affected by tourists than the more famous Bunaken National Park (BNP), Lembeh Strait, or other Indonesian islands, such as Bali. However, implementation of mining operations began in 2012 in coastal areas, leading to deforestation of mangroves and broad land erosion within only 5 years. Unfortunately, there are no detailed data available for the diversity of marine organisms and coral reef health in Bangka Island before mining started; therefore, the impacts of the mining in areas close by cannot be assessed. This study therefore aims to serve as a baseline for future monitoring surveys concerning further environmental changes within the Bangka Archipelago.

\section{Materials and Methods}

Material was collected during the dry season, in September 2017 and September 2018. In total, 28 dives were performed during the day and 10 during the early night, with 3-5 divers and 60-120 minutes per dive in 19 different localities around Bangka Island and the adjacent mainland (Figure 1A,B). A depth range from the eulittoral down to ca. $20 \mathrm{~m}$ was covered. Table 1 summarizes the specific conditions of the various diving localities. Various substrates, such as macro algae and coral rubble, were collected for further analyses in the laboratory. Additionally, several hours were spent collecting and photo-documenting while snorkeling. In a few sites, only underwater photographs were taken of sea slugs for species recording. These species are included in our results on species numbers, but not in our overall specimen numbers.

Each collected specimen was documented with an Olympus TG5 under water (when possible) and additionally in the laboratory, provided with a unique identifier (abbreviation of name, year, location, and number of specimen), and preliminarily identified with the help of available literature (e.g., [3-5,11,15,16,20-27]). Gosliner et al. [24] depict many undescribed species. We used their species number for our internal identifier when we assigned one of our specimens to these undescribed species. However, we applied only a letter (e.g., sp. a, or sp. b) when a specific assignment to any undescribed species in this identification book was not possible and/or when our barcodes checked against the reference database in NCBI (National Center for Biotechnology Information) provided no hit. Validity of names was checked in the World Register of Marine Species [26]. Collected specimens were preserved in $96 \% \mathrm{EtOH}$ and/or in formaldehyde/seawater. 

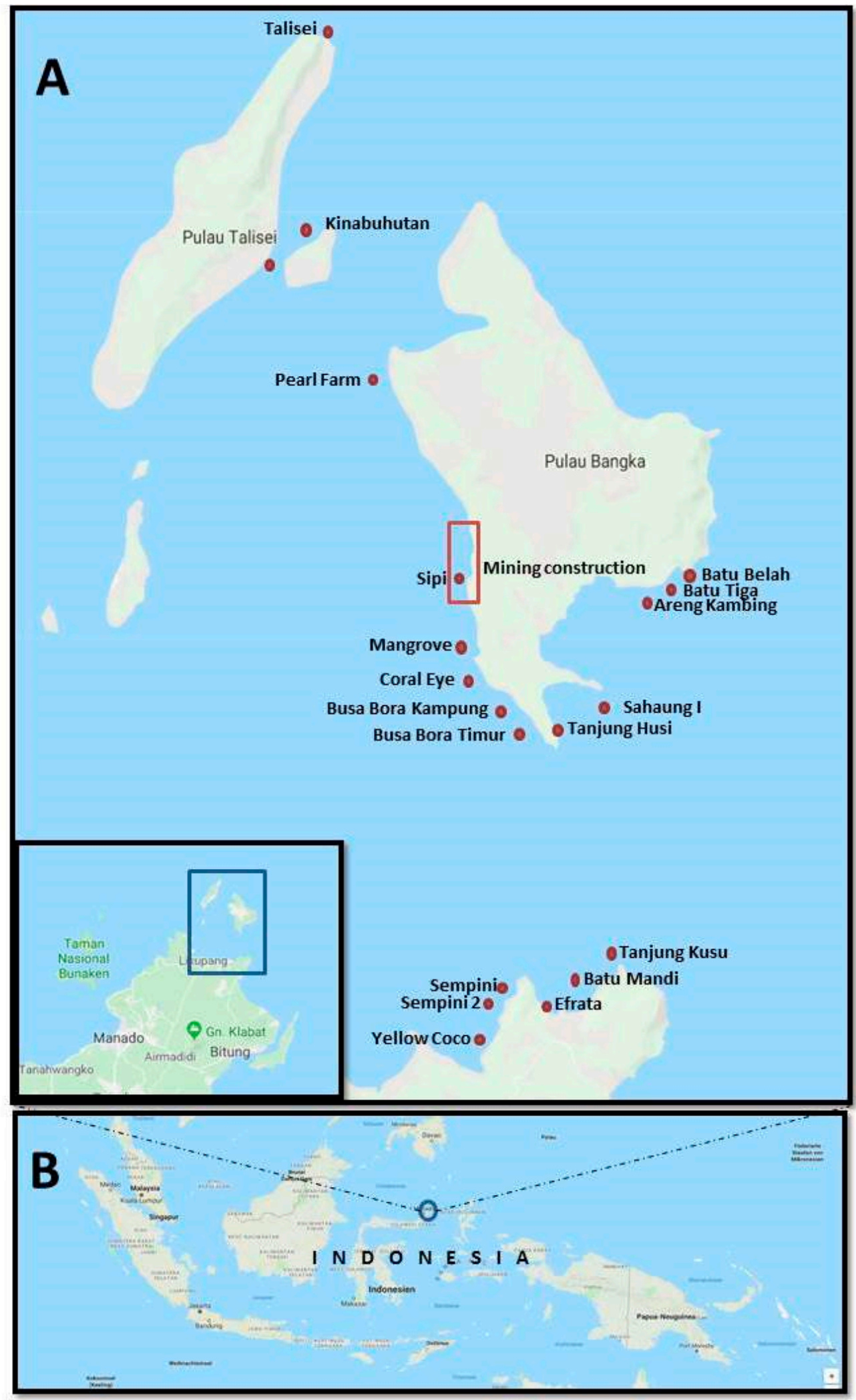

Figure 1. Location of the study area: (A) depiction of the 19 diving sites at Bangka Archipelago and mainland. Insert showing the region of North Sulawesi with Bangka Archipelago. (B) Indonesia and Sulawesi, with the blue circle indicating the close-up area in (A). 
Table 1. Information about collection sites and dates in 2017-2018. * Batu Tiga is the only locality where no samples were collected, and specimens were recorded only by photo-documentation provided by Gianni Valenti.

\begin{tabular}{|c|c|c|c|}
\hline $\begin{array}{c}\text { Area of } \\
\text { Collection Site }\end{array}$ & Location & Characteristics of the Habitat & $\begin{array}{l}\text { Year of } \\
\text { Collection }\end{array}$ \\
\hline \multicolumn{4}{|c|}{ Bangka Archipelago } \\
\hline Batu Belah & $\begin{array}{c}1^{\circ} 46^{\prime} 22.20^{\prime \prime} \mathrm{N} \\
125^{\circ} 10^{\prime} 57.38^{\prime \prime} \mathrm{E}\end{array}$ & $\begin{array}{l}\text { Sandy bottom with a deposit of large } \\
\text { formations of stones richly covered with corals }\end{array}$ & 2017-2018 \\
\hline Batu Tiga * & $\begin{array}{l}1^{\circ} 46^{\prime} 07.6^{\prime \prime} \mathrm{N} \\
125^{\circ} 10^{\prime} 35.7^{\prime \prime} \mathrm{E}\end{array}$ & Coral pinnacles on a deep coral sand bottom & 2018 \\
\hline Areng Kambing & $\begin{array}{c}1^{\circ} 46^{\prime} 07.8^{\prime \prime} \mathrm{N} \\
125^{\circ} 10^{\prime} 34.6^{\prime \prime} \mathrm{E}\end{array}$ & Coral sand and coral rabble slope & 2018 \\
\hline Sahaung & $\begin{array}{l}1^{\circ} 44^{\prime} 35.11^{\prime \prime} \mathrm{N} \\
125^{\circ} 09^{\prime} 43.5^{\prime \prime} \mathrm{E}\end{array}$ & $\begin{array}{c}\text { A shallow area made up of rocks and pinnacles } \\
\text { of volcanic origin, richly covered with } \\
\text { coral formations }\end{array}$ & 2017 \\
\hline Tanjung Husi & $\begin{array}{c}1^{\circ} 44^{\prime} 07.74^{\prime \prime} \mathrm{N} \\
125^{\circ} 09^{\prime} 05.59^{\prime \prime} \mathrm{E}\end{array}$ & $\begin{array}{c}\text { Volcanic rockslide, characterized by the } \\
\text { presence of strong tidal currents and } \\
\text { ocean swell }\end{array}$ & 2017-2018 \\
\hline Busa Bora Timur & $\begin{array}{l}1^{\circ} 44^{\prime} 27.4^{\prime \prime} \mathrm{N} \\
125^{\circ} 08^{\prime} 35.1^{\prime \prime} \mathrm{E}\end{array}$ & Fringing reef on a coral sand slope & 2018 \\
\hline $\begin{array}{l}\text { Busa Bora } \\
\text { Kampung }\end{array}$ & $\begin{array}{c}1^{\circ} 44^{\prime} 39.8^{\prime \prime} \mathrm{N} \\
125^{\circ} 08^{\prime} 24.4^{\prime \prime} \mathrm{E}\end{array}$ & $\begin{array}{l}\text { Fringing reef on a coral sand slope in front of a } \\
\text { little village }\end{array}$ & 2018 \\
\hline Coral Eye & $\begin{array}{c}1^{\circ} 45^{\prime} 03.61^{\prime \prime} \mathrm{N} \\
125^{\circ} 07^{\prime} 58.85^{\prime \prime} \mathrm{E}\end{array}$ & $\begin{array}{l}\text { Fringing reef in front of a resort; } 2012 \text { was the } \\
\text { last storm [1]. Dominant branching coral and } \\
\text { little rubble. Coral grown over the tide surface. }\end{array}$ & 2017-2018 \\
\hline Mangrove & $\begin{array}{c}1^{\circ} 45^{\prime} 46.9^{\prime \prime} \mathrm{N} \\
125^{\circ} 07^{\prime} 50.0^{\prime \prime} \mathrm{E}\end{array}$ & $\begin{array}{l}\text { Fringing reef on a coral sand slope that starts } \\
\text { from the lagoon in front of the mangrove }\end{array}$ & 2017-2018 \\
\hline Sipi & $\begin{array}{c}1^{\circ} 47^{\prime} 02.8^{\prime \prime} \mathrm{N} \\
125^{\circ} 07^{\prime} 42.4^{\prime \prime} \mathrm{E}\end{array}$ & $\begin{array}{l}\text { River mouth: mud/fringing reef, site impacted } \\
\text { by mining operations }\end{array}$ & 2018 \\
\hline Pearl Farm & $\begin{array}{c}1^{\circ} 48^{\prime} 41.3^{\prime \prime} \mathrm{N} \\
125^{\circ} 06^{\prime} 45.1^{\prime \prime} \mathrm{E}\end{array}$ & $\begin{array}{l}\text { Former abandoned pearl farm, fringing reef on } \\
\text { a coral sand slope }\end{array}$ & 2018 \\
\hline Kinabuhutan & $\begin{array}{l}1^{\circ} 50^{\prime} 45.44^{\prime \prime} \mathrm{N} \\
125^{\circ} 5^{\prime} 55.26^{\prime \prime} \mathrm{E}\end{array}$ & Fringing reef on a shallow coral sand slope & 2018 \\
\hline Talisei & $\begin{array}{c}1^{\circ} 53^{\prime} 38.32^{\prime \prime} \mathrm{N} \\
125^{\circ} 05^{\prime} 53.82^{\prime \prime} \mathrm{E}\end{array}$ & $\begin{array}{l}\text { A vertical wall of volcanic rock that ends on a } \\
\text { sandy bottom. The spot is subject to strong } \\
\text { tidal currents and ocean swell, so the coral } \\
\text { formations are small and compact. }\end{array}$ & 2018 \\
\hline \multicolumn{4}{|l|}{ Mainland } \\
\hline Yellow Coco & $\begin{array}{l}1^{\circ} 40^{\prime} 21.67^{\prime \prime} \mathrm{N} \\
125^{\circ} 8^{\prime} 6.60^{\prime \prime} \mathrm{E}\end{array}$ & $\begin{array}{l}\text { Fringing reef on a mix of volcanic and coral } \\
\text { sand slopes }\end{array}$ & 2018 \\
\hline Sempini & $\begin{array}{l}1^{\circ} 41^{\prime} 4.83^{\prime \prime} \mathrm{N} \\
125^{\circ} 8^{\prime} 32.65^{\prime \prime} \mathrm{E}\end{array}$ & Fringing reef on a coral sand slope & 2018 \\
\hline Sempini 2 & $\begin{array}{l}1^{\circ} 40^{\prime} 51.06^{\prime \prime} \mathrm{N} \\
125^{\circ} 8^{\prime} 22.45^{\prime \prime} \mathrm{E}\end{array}$ & Fringing reef on a coral sand slope & 2018 \\
\hline Efrata & $\begin{array}{l}1^{\circ} 40^{\prime} 52.34^{\prime \prime} \mathrm{N} \\
125^{\circ} 9^{\prime} 10.55^{\prime \prime} \mathrm{E}\end{array}$ & Volcanic rock beach, followed by volcanic sand & 2018 \\
\hline Batu Mandi & $\begin{array}{c}1^{\circ} 41^{\prime} 16.71^{\prime \prime} \mathrm{N} \\
125^{\circ} 09^{\prime} 42.79^{\prime \prime} \mathrm{E}\end{array}$ & $\begin{array}{l}\text { Volcanic rock covered by coral reef, sponge, } \\
\text { and other invertebrates. Various topographies. }\end{array}$ & 2017-2018 \\
\hline $\begin{array}{c}\text { Tanjung } \\
\text { Kusu-Kusu }\end{array}$ & $\begin{array}{c}1^{\circ} 41^{\prime} 23.01^{\prime \prime} \mathrm{N} \\
125^{\circ} 09^{\prime} 55.26^{\prime \prime} \mathrm{E}\end{array}$ & $\begin{array}{l}\text { Volcanic rock wall on a backdrop of rock and } \\
\text { coral, characterized by the presence of strong } \\
\text { tidal currents and ocean swell }\end{array}$ & 2017 \\
\hline
\end{tabular}


Those families with documented cryptic speciation (e.g., Chromodorididae, Phyllidiidae) and a few other rare species of interest (e.g., Moridilla) were analyzed by using barcoding methods to verify identification. Additionally, small specimens that were difficult to identify (if at all) or where no clear assignment was possible were also barcoded.

All specimens are registered in the collection of Sam Ratulangi University according to the year under the numbers SRU2017/01 and SRU2018/01. Metadata of each individual are documented in the database Diversity Collection (Diversity Workbench) using the data brokerage service of the German Federation for Biological Data (https://www.gfbio.org/) [28]. Data are publicly available at www.gfbio.org for browsing and the archived data can be downloaded at https://doi.org/10.20363/ heterobranchia-bangka-prj-1.1.

\section{Results}

In total, 484 specimens comprising 149 species of marine heterobranchs were collected in the two sampling events in 2017 and 2018. Five species can be assigned to Cephalaspidea, two to Aplysiida, 15 to Sacoglossa, two to Pleurobranchida, and 124 to Nudibranchia. Additionally, one species is a member of the eupulmonate taxon Onchidiidae. Thirteen species of the 149 were only recorded by photo-documentation in 2018 without collection. Thirty-three species cannot be assigned to any described species and are considered new species. Table 2 summarizes available information on all collected and photo-documented specimens, and also includes information about taxa authorities (these are therefore not mentioned in the text). Families and species are listed in the same order as in the text below. The table also provides information about the presence of the particular species in BNP, Sangihe, and Lembeh Strait according to the recently published surveys [16-18] to highlight those species that are only recorded from our study area. All species listed in Table 2 are also depicted by at least one specimen, together with the specimen identifier, if the animal was collected, and are available for further investigations. In the following sections, species and specimens are discussed and compared to other studies from North Sulawesi [1,16-18].

\subsection{Animal Collections}

\subsubsection{Cephalaspidea (Five Species in Five Genera Belonging to Four Families)}

The number of cephalaspidean species is low in comparison to the number from BNP (5 vs. 16).

Haminoeidae: Haminoea sp. (Figure 2A), collected at a depth of $7 \mathrm{~m}$, has a translucent orange shell with orange and yellow marks, resembling Haminoea sp. 3 from Gosliner et al. [24].

Colpodaspidae: Colpodaspis thompsoni (Figure 2B; length 2-5 mm) was most often found crawling in the coral rubble at 1.5-9.2 m depth. With only five specimens, it was not as common as in BNP.

Aglajidae: Only two specimens of Chelidonura amoena (Figure 2C), a common species in sandy reefal habitats, were found in one site only. One specimen of Odontoglaja (Odsp.a18Ba-1, Figure 2D,E) was found in the coral rubble. It looks similar to Odontoglaja guamensis but lacks the distinct color pattern on the dorsum. Gosliner et al. [24] depict several Odontoglaja spp. with similar background patterns; however, the dorsal color patterns and especially the intensity of the color differ in all of these. We cannot assign our specimen to any of these taxa.

Gastropteridae: Two specimens of Sagaminopteron psychedelicum (Figure 2F) were found in coral rubble. 
Table 2. List of species, specimen identifiers, and further metadata of the material; $\left({ }^{*}\right)$ indicate species that were identified only by photo-documentation. The last three columns indicate the number of specimens or occurrence of the respective species in other study areas nearby (information is taken from Eisenbarth et al. (2018) [16], Undap et al. (2019) [18], and Ompi et al. (2019) [17]: BNP, Lembeh Strait, and Sangihe Island).

\begin{tabular}{|c|c|c|c|c|c|c|c|c|c|c|c|c|c|c|c|c|c|c|c|c|c|}
\hline \multirow[b]{3}{*}{$\begin{array}{l}\text { Higher Taxon } \\
\text { Affiliation }\end{array}$} & \multicolumn{18}{|c|}{ Expedition in Bangka Archipelago and Mainland Nearby } & \multicolumn{3}{|c|}{$\begin{array}{c}\text { Other } \\
\text { Expedition }\end{array}$} \\
\hline & \multirow[b]{2}{*}{ Identifier } & \multirow[b]{2}{*}{ Species Name } & \multirow[b]{2}{*}{ 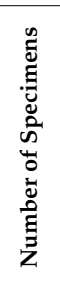 } & \multirow[b]{2}{*}{$\begin{array}{l}\bar{E} \\
\text { हु } \\
\stackrel{\tilde{N}}{\omega}\end{array}$} & \multirow[b]{2}{*}{$\begin{array}{l}\widehat{\Xi} \\
\bar{\Xi} \\
\overline{0} \\
\text { D̆ }\end{array}$} & \multicolumn{9}{|c|}{ Bangka Archipelago } & \multicolumn{4}{|c|}{ Mainland } & \multirow[b]{2}{*}{ 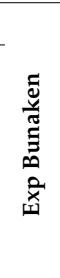 } & \multirow[b]{2}{*}{ 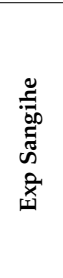 } & \multirow[b]{2}{*}{ 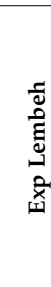 } \\
\hline & & & & & & 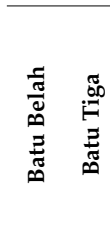 & 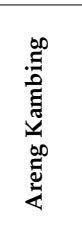 & 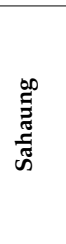 & 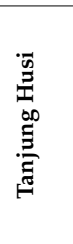 & 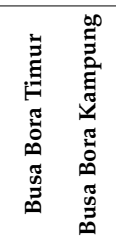 & 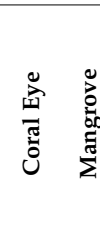 & के & $\begin{array}{l}\text { E్ } \\
\text { 焉 } \\
\stackrel{\Xi}{\Xi}\end{array}$ & 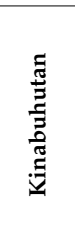 & 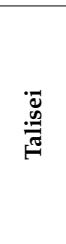 & 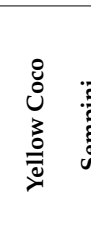 & 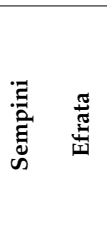 & 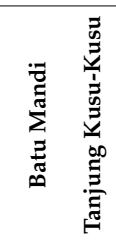 & & & \\
\hline \multicolumn{22}{|c|}{ Cephalaspidea ( 5 species) } \\
\hline $\begin{array}{l}\text { Haminoeidae Pilsbry, } \\
1895\end{array}$ & Hasp18Ba-1 & $\begin{array}{l}\text { Haminoea sp. (Haminoea sp. } 3 \\
\text { in Gosliner et al. [24]: 30) }\end{array}$ & 1 & 2 & 7 & & & & & & & & & & 1 & & & & $\mathrm{x}$ & - & - \\
\hline $\begin{array}{l}\text { Colpodaspididae } \\
\text { Oskars, Bouchet and } \\
\text { Malaquias, } 2015\end{array}$ & & $\begin{array}{l}\text { Colpodaspis thompsoni Brown, } \\
1979\end{array}$ & 5 & $2-5$ & $1.5-9.2$ & & & & 2 & 1 & 1 & & 1 & & & & & & $\mathrm{x}$ & - & - \\
\hline \multirow{2}{*}{$\begin{array}{c}\text { Aglajidae } \\
\text { Pilsbry, } 1895 \text { (1847) }\end{array}$} & & Chelidonura amoena Bergh, 1905 & 2 & $11-25$ & $4-9.1$ & & & & & & 2 & & & & & & & & $\mathrm{x}$ & - & - \\
\hline & Odsp.a18Ba-1 & Odontoglaja sp. a & 1 & 15 & 1.5 & & & & & & 1 & & & & & & & & - & - & - \\
\hline $\begin{array}{l}\text { Gastropteridae } \\
\text { Swainson, } 1840\end{array}$ & & $\begin{array}{l}\text { Sagaminopteron psychedelicum } \\
\text { Carlson and Hoff, } 1974\end{array}$ & 2 & 3 & 1 & & & & & & 1 & & & & & & & & $\mathrm{x}$ & - & - \\
\hline \multicolumn{22}{|c|}{ Aplysiida (2 species) } \\
\hline \multirow{2}{*}{$\begin{array}{l}\text { Aplysiidae } \\
\text { Lamarck, } 1809\end{array}$} & & $\begin{array}{l}\text { Aplysia cf. nigrocincta von } \\
\text { Martens, } 1880\end{array}$ & 4 & $4-10$ & 1 & & & & & & 4 & & & & & & & & $\mathrm{x}$ & - & - \\
\hline & & $\begin{array}{l}\text { Stylocheilus striatus (Quoy and } \\
\text { Gaimard, 1832) }\end{array}$ & 2 & $5-15$ & $7-7.5$ & & & & & & 1 & & & & & & 1 & & $\mathrm{x}$ & - & - \\
\hline \multicolumn{22}{|c|}{ Sacoglossa (15 species) } \\
\hline $\begin{array}{c}\text { Oxynoidae } \\
\text { Stoliczka, } 1868 \text { (1847) } \\
\end{array}$ & $\begin{array}{l}\text { Losp1-18Ba1; } \\
\text { Losp1-18Ba2 }\end{array}$ & $\begin{array}{l}\text { Lobiger sp. (Lobiger sp. } 1 \text { in } \\
\text { Gosliner et al. [24]: 70) }\end{array}$ & 2 & $2-11$ & 8.4 & & & & 2 & & & & & & & & & & $\mathrm{x}$ & - & - \\
\hline \multirow{2}{*}{$\begin{array}{l}\text { Hermaeidae } \mathrm{H} . \\
\text { Adams and A. } \\
\text { Adams, } 1854\end{array}$} & & Cyerce bourbonica Yonow, 2012 & 2 & $3-4$ & $1-1.5$ & & & & & & 2 & & & & & & & & $\mathrm{x}$ & - & - \\
\hline & & Cyerce nigra Bergh, 1871 & 1 & 4 & 8.6 & & & & 1 & & & & & & & & & & - & - & - \\
\hline
\end{tabular}


Table 2. Cont.

\begin{tabular}{|c|c|c|c|c|c|c|c|c|c|c|c|c|c|c|c|c|c|c|c|c|c|c|c|c|}
\hline \multirow[b]{3}{*}{$\begin{array}{l}\text { Higher Taxon } \\
\text { Affiliation }\end{array}$} & \multicolumn{21}{|c|}{ Expedition in Bangka Archipelago and Mainland Nearby } & \multicolumn{3}{|c|}{$\begin{array}{c}\text { Other } \\
\text { Expeditions }\end{array}$} \\
\hline & \multirow[b]{2}{*}{ Identifier } & \multirow[b]{2}{*}{ Species Name } & \multirow[b]{2}{*}{ 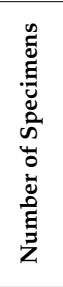 } & \multirow[b]{2}{*}{$\begin{array}{l}\overline{\mathbf{g}} \\
\stackrel{\tilde{N}}{\tilde{n}}\end{array}$} & \multirow[b]{2}{*}{$\begin{array}{l}\overline{\mathrm{g}} \\
\overline{\bar{z}} \\
\text { 口̆ }\end{array}$} & \multicolumn{11}{|c|}{ Bangka Archipelago } & \multicolumn{5}{|c|}{ Mainland } & \multirow[b]{2}{*}{ 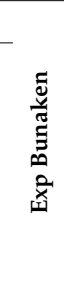 } & \multirow[b]{2}{*}{ 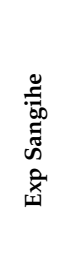 } & \multirow[b]{2}{*}{ 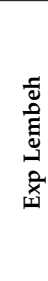 } \\
\hline & & & & & & 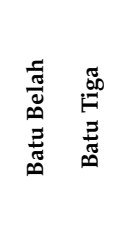 & 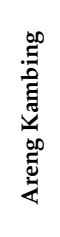 & 先 & 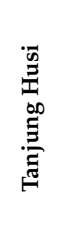 & 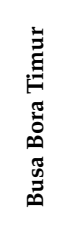 & 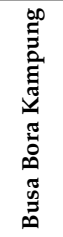 & $\begin{array}{l}\text { 总 } \\
\text { 范 }\end{array}$ & 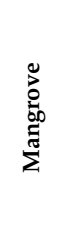 & 产 & 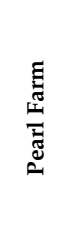 & 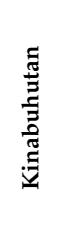 & 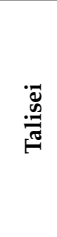 & $\begin{array}{l}8 \\
0 \\
3 \\
0 \\
\bar{\Xi}\end{array}$ & 咅 & 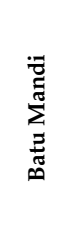 & 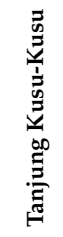 & & & \\
\hline \multirow{13}{*}{$\begin{array}{l}\text { Plakobranchidae } \\
\text { Gray, } 1840\end{array}$} & & $\begin{array}{l}\text { Elysia asbecki Wägele, Stemmer, } \\
\text { Burghardt and Händeler, } 2010\end{array}$ & 1 & 3 & 8.5 & & & & 1 & & & & & & & & & & & & & $\mathrm{x}$ & - & - \\
\hline & & Elysia marginata (Pease, 1871) & 1 & 3 & 11.6 & & & & & & & & & & & & & & & & 1 & $\mathrm{x}$ & - & - \\
\hline & & $\begin{array}{l}\text { Elysia cf. nigropunctata (Pease, } \\
\text { 1871) }\end{array}$ & 1 & 21 & 2.6 & & & & & & & 1 & & & & & & & & & & - & - & - \\
\hline & & Elysia pusilla (Bergh, 1871) & 2 & $7-11$ & $1-1.5$ & & & & & & & 1 & 1 & & & & & & & & & $\mathrm{x}$ & $\mathrm{x}$ & - \\
\hline & Elsp24-18Ba-1 & $\begin{array}{l}\text { Elysia sp. } 24 \text { (in Gosliner } \\
\text { et al. [24]: 89) }\end{array}$ & 1 & 7 & 7 & & & & & & & 1 & & & & & & & & & & - & - & - \\
\hline & Elsp27-18Ba-1 & $\begin{array}{l}\text { Elysia sp. } 27 \text { (in Gosliner } \\
\text { et al. [24]: 89) }\end{array}$ & 1 & 5 & 7 & & & & & & & 1 & & & & & & & & & & - & - & - \\
\hline & Elsp.a18Ba-1 & Elysia sp. a & 1 & 8 & 1 & & & & & & & & 1 & & & & & & & & & $\mathrm{x}$ & - & - \\
\hline & Elsp.b18Ba-1 & Elysia sp. b & 1 & 9 & 20 & & & & & & & & & & & & & 1 & & & & $\mathrm{x}$ & - & - \\
\hline & & $\begin{array}{l}\text { Thuridilla carlsoni Gosliner, } \\
1995\end{array}$ & 2 & $10-17$ & $7.6-8.3$ & 1 & & & & & & & & & & & & & 1 & & & - & - & - \\
\hline & & $\begin{array}{l}\text { Thuridilla flavomaculata } \\
\text { Gosliner, } 1995\end{array}$ & 1 & 5 & 5.3 & & & & 1 & & & & & & & & & & & & & $\mathrm{x}$ & - & - \\
\hline & & Thuridilla vataae (Risbec, 1928) & 2 & $7-9$ & $1.5-3$ & & & & & & & 2 & & & & & & & & & & $\mathrm{x}$ & - & - \\
\hline & & Thuridilla gracilis (Risbec, 1928) & 12 & $8-24$ & $1-12.8$ & 1 & & & 2 & & 3 & 4 & 1 & 1 & & & & & & & & - & $\mathrm{x}$ & - \\
\hline & & \multicolumn{23}{|c|}{ Pleurobranchida (2 species) } \\
\hline \multirow{2}{*}{$\begin{array}{l}\text { Pleurobranchidae } \\
\text { Gray, } 1827\end{array}$} & & $\begin{array}{c}\text { Pleurobranchus forskalii } \\
\text { Rüppell and Leuckart, } 1828\end{array}$ & 2 & $23-25$ & $4-12$ & & & & & & 1 & 1 & & & & & & & & & & $\mathrm{x}$ & - & - \\
\hline & & $\begin{array}{l}\text { Pleurobranchus peronii Cuvier, } \\
1804\end{array}$ & 2 & 19-140 & $2-12$ & & & & & & 1 & 1 & & & & & & & & & & - & - & - \\
\hline \multicolumn{25}{|c|}{ Nudibranchia, Doridina (82 species) } \\
\hline $\begin{array}{l}\text { Hexabranchidae } \\
\text { Bergh, } 1891\end{array}$ & Glsp1_17Ba-1 & $\begin{array}{l}\text { Hexabranchus sanguineus } \\
\text { (Rüppell and Leuckart, 1830) }\end{array}$ & 1 & 10 & 6.6 & & & & 1 & & & & & & & & & & & & & $\mathrm{x}$ & - & - \\
\hline
\end{tabular}


Table 2. Cont.

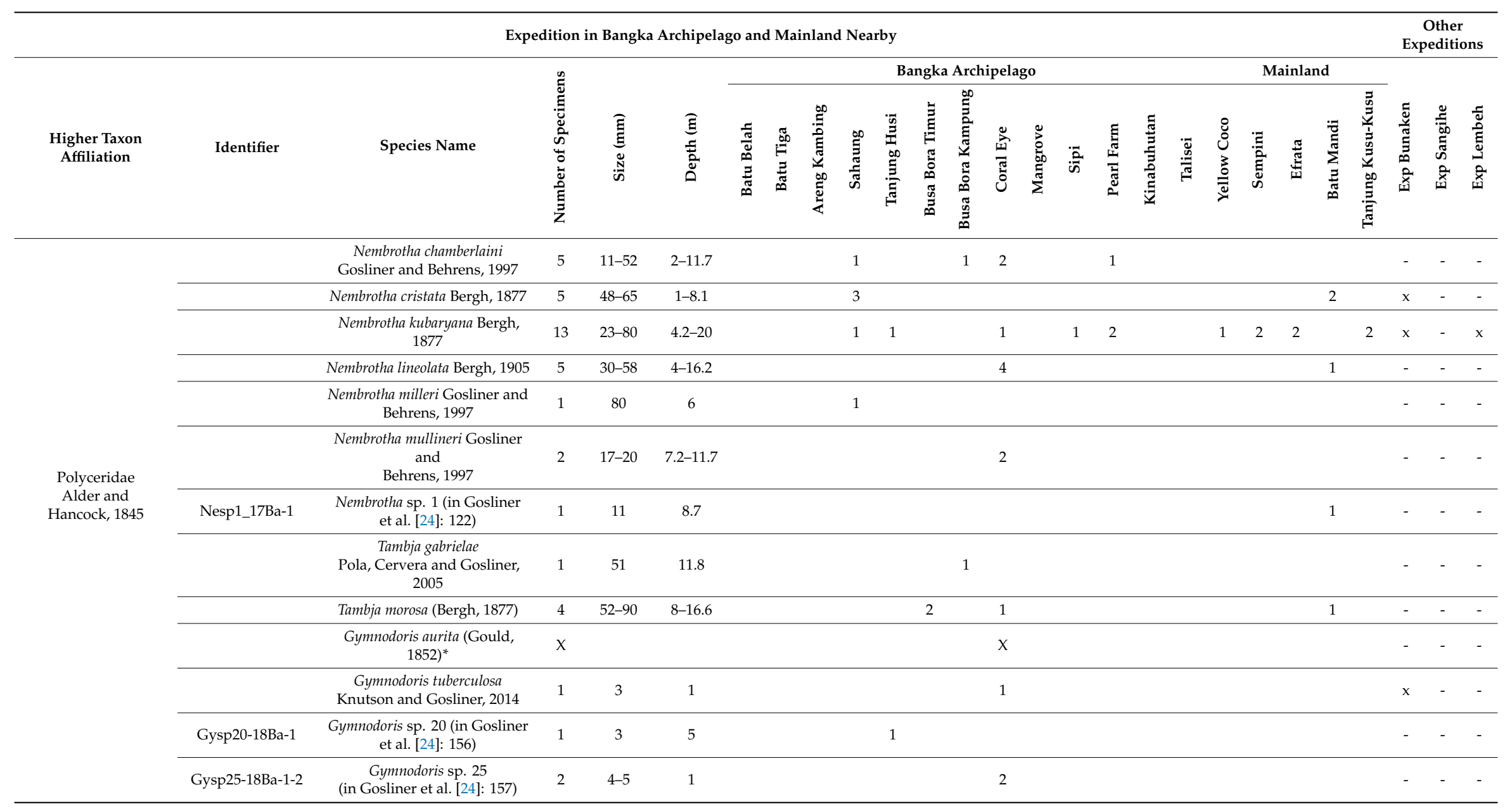


Table 2. Cont.

\begin{tabular}{|c|c|c|c|c|c|c|c|c|c|c|c|c|c|c|c|c|c|c|c|c|c|c|c|c|c|c|}
\hline \multirow[b]{3}{*}{$\begin{array}{l}\text { Higher Taxon } \\
\text { Affiliation }\end{array}$} & \multirow[b]{3}{*}{ Identifier } & \multicolumn{22}{|c|}{ Expedition in Bangka Archipelago and Mainland Nearby } & \multicolumn{3}{|c|}{$\begin{array}{c}\text { Other } \\
\text { Expeditions }\end{array}$} \\
\hline & & \multirow[b]{2}{*}{ Species Name } & \multirow[b]{2}{*}{ 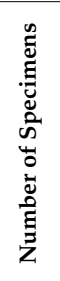 } & \multirow[b]{2}{*}{ 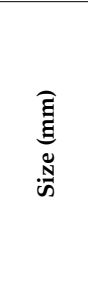 } & \multirow[b]{2}{*}{ 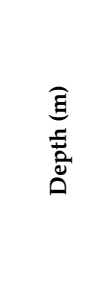 } & \multicolumn{12}{|c|}{ Bangka Archipelago } & \multicolumn{6}{|c|}{ Mainland } & \multirow[b]{2}{*}{ 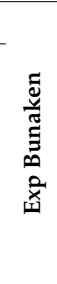 } & \multirow[b]{2}{*}{ 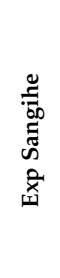 } & \multirow[b]{2}{*}{ 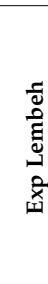 } \\
\hline & & & & & & 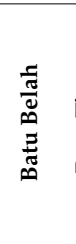 & 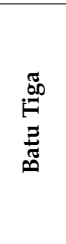 & 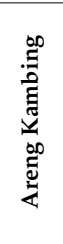 & 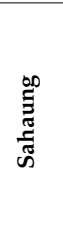 & 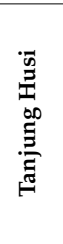 & 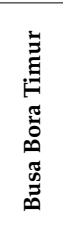 & 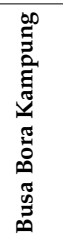 & $\begin{array}{l}\text { 离 } \\
\text { 苛 }\end{array}$ & 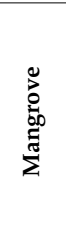 & के & 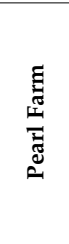 & 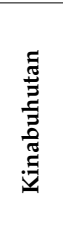 & 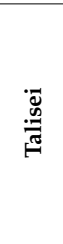 & $\begin{array}{l}8 \\
\ddot{\Xi} \\
3 \\
\stackrel{0}{\bar{\Xi}}\end{array}$ & 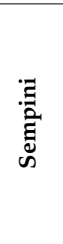 & 莺 & 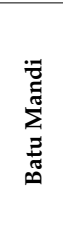 & 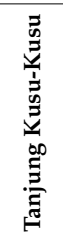 & & & \\
\hline \multirow{3}{*}{$\begin{array}{l}\text { Goniodorididae } \\
\text { H. Adams and A. } \\
\text { Adams, } 1854\end{array}$} & Gosp7-18Ba-1 & $\begin{array}{l}\text { Goniodoris sp. } 7 \text { (in Gosliner } \\
\text { et al. [24]: 153) }\end{array}$ & 1 & 6 & 10 & & & & & & & & 1 & & & & & & & & & & & - & - & - \\
\hline & & $\begin{array}{l}\text { Trapania armilla Gosliner and } \\
\text { Fahey, } 2008\end{array}$ & 1 & 7 & & & & & & & & & 1 & & & & & & & & & & & - & - & - \\
\hline & & Trapania safracornia Fahey, 2004 & 1 & 7 & 13.4 & & & & & & & 1 & & & & & & & & & & & & - & - & - \\
\hline \multirow{2}{*}{$\begin{array}{l}\text { Aegiridae } \\
\text { P. Fischer, } 1883\end{array}$} & Aesp7-18Ba-1 & $\begin{array}{l}\text { Aegires sp. } 7 \text { (in Gosliner } \\
\quad \text { et al. [24]: 149) }\end{array}$ & 1 & 4 & 7 & & & & & & & & & 1 & & & & & & & & & & - & - & - \\
\hline & & Notodoris minor Eliot, $1904 *$ & $x$ & & & & $x$ & & & & & & & & & & & & & & & & & - & - & - \\
\hline \multirow{9}{*}{$\begin{array}{l}\text { Discodorididae } \\
\text { Bergh, } 1891\end{array}$} & & $\begin{array}{l}\text { Asteronotus cespitosus (van } \\
\text { Hasselt, 1824)* }\end{array}$ & $\mathrm{x}$ & & & & & & & & & & $\mathrm{x}$ & & & & & & & & & & & - & - & - \\
\hline & & $\begin{array}{l}\text { Asteronotus mimeticus Gosliner } \\
\text { and Valdés, } 2002\end{array}$ & 9 & $5-25$ & 3-11.9 & & & & & & & & 8 & & & & & & & 1 & & & & $\mathrm{x}$ & - & - \\
\hline & & Atagema intecta (Kelaart, 1859) & 2 & $12-55$ & $7-8$ & & & & & & & & 2 & & & & & & & & & & & - & - & - \\
\hline & & Discodoris cebuensis Bergh, 1877 & 2 & $48-53$ & 11.2 & & & & & & & & 2 & & & & & & & & & & & - & - & - \\
\hline & & $\begin{array}{c}\text { Halgerda batangas Carlson and } \\
\text { Hoff, } 2000\end{array}$ & 10 & $32-57$ & $6.2-19.2$ & 1 & & & & & & & & & 1 & & & & 2 & 5 & 1 & & & $\mathrm{x}$ & - & $\mathrm{x}$ \\
\hline & & Halgerda carlsoni Rudman, 1978 & 2 & $33-40$ & $11.8-12$ & & & & & & & & & & & & & & & & & 1 & 1 & $\mathrm{x}$ & - & \\
\hline & & Jorunna funebris Kelaart, 1859 & 2 & $15-20$ & 2 & & & & & & & & & 2 & & & & & & & & & & - & - & $x$ \\
\hline & & Paradoris liturata (Bergh, 1905) & 3 & $22-43$ & $8-8.5$ & & & & & & & 1 & & & & & & & & & 1 & & 1 & - & - & - \\
\hline & & Platydoris sanguinea Bergh, 1905 & 1 & 12 & 5.5 & & & & & & & & 1 & & & & & & & & & & & $\mathrm{x}$ & - & - \\
\hline
\end{tabular}


Table 2. Cont.

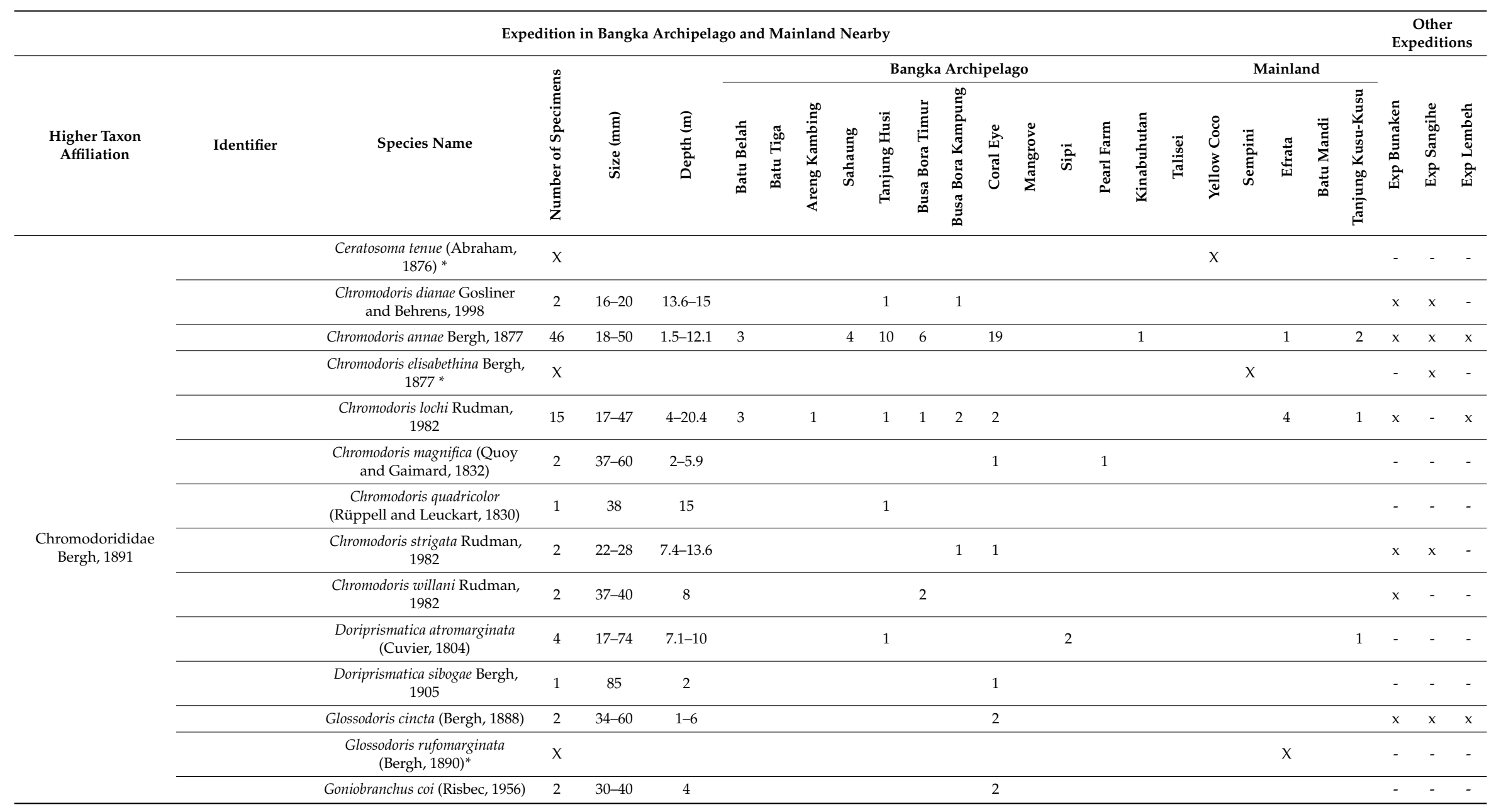


Table 2. Cont.

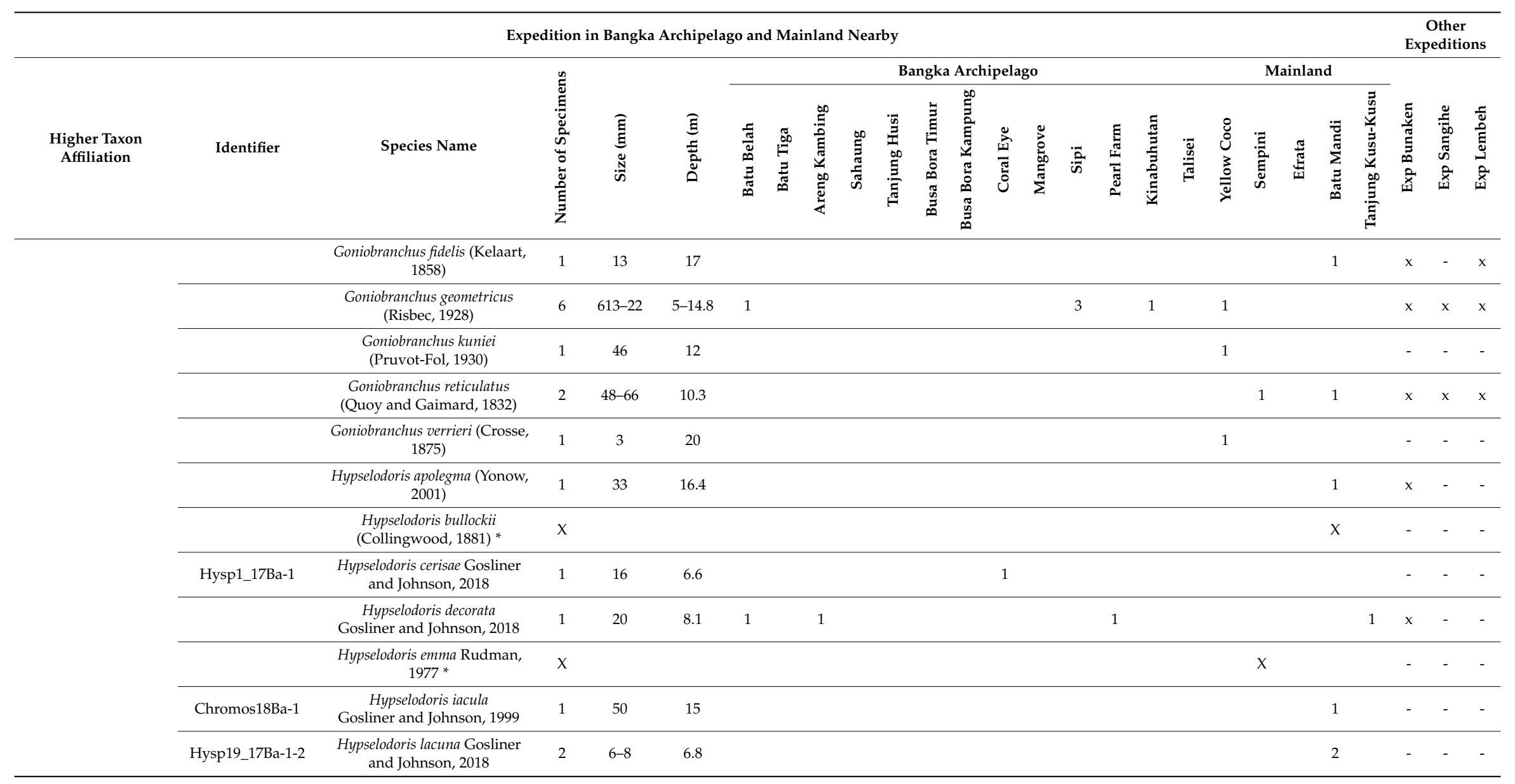


Table 2. Cont.

\begin{tabular}{|c|c|c|c|c|c|c|c|c|c|c|c|c|c|c|c|c|c|c|c|c|c|c|c|c|c|c|}
\hline \multirow[b]{3}{*}{$\begin{array}{l}\text { Higher Taxon } \\
\text { Affiliation }\end{array}$} & \multicolumn{23}{|c|}{ Expedition in Bangka Archipelago and Mainland Nearby } & \multicolumn{3}{|c|}{$\begin{array}{c}\text { Other } \\
\text { Expedition }\end{array}$} \\
\hline & & \multirow{2}{*}{\multicolumn{2}{|c|}{ 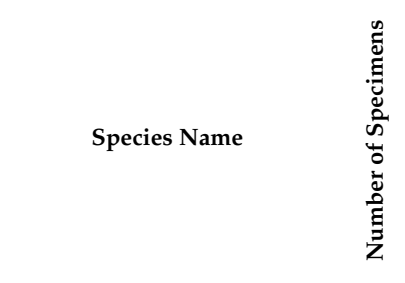 }} & \multirow[b]{2}{*}{ 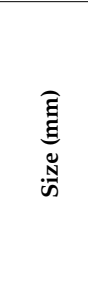 } & \multirow[b]{2}{*}{ 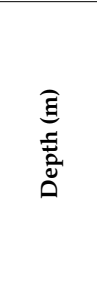 } & \multicolumn{12}{|c|}{ Bangka Archipelago } & \multicolumn{6}{|c|}{ Mainland } & \multirow[b]{2}{*}{ 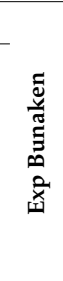 } & \multirow[b]{2}{*}{ 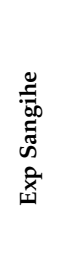 } & \multirow[b]{2}{*}{ 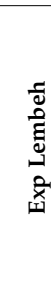 } \\
\hline & Identifier & & & & & 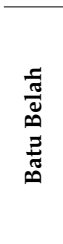 & 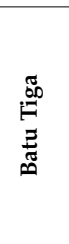 & 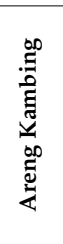 & 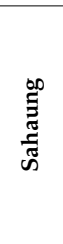 & 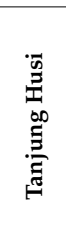 & 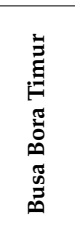 & 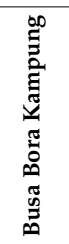 & 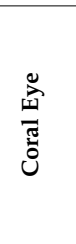 & 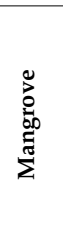 & $\vec{n}$ & 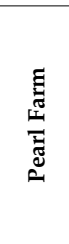 & 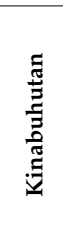 & 总 & $\begin{array}{l}\stackrel{8}{0} \\
3 \\
3 \\
\stackrel{0}{\bar{J}}\end{array}$ & 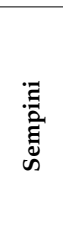 & 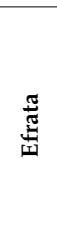 & 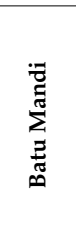 & 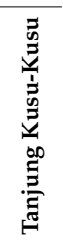 & & & \\
\hline & & $\begin{array}{c}\text { Hypselodoris maculosa (Pease, } \\
1871 \text { ) } \\
\end{array}$ & 4 & $18-23$ & $3-10.3$ & 1 & & & & & & & & & & & & & & & & & & - & - & - \\
\hline & & $\begin{array}{l}\text { Hypselodoris maridadilus } \\
\text { Rudman, } 1977\end{array}$ & 1 & 10 & 6.5 & & & & & 1 & & & & & & & & & & & & & & - & - & - \\
\hline & & $\begin{array}{l}\text { Hypselodoris zephyra (Eliot, } \\
\text { 1904) }\end{array}$ & 1 & 9 & 14.2 & 1 & & & & & & & & & & & & & & & & & & - & - & - \\
\hline & & $\begin{array}{l}\text { Hypselodoris tryoni (Garret } \\
\text { 1873) }\end{array}$ & 7 & $26-54$ & $4-14.1$ & & & & & & & 2 & & & 2 & & & & & & 2 & 1 & & $\mathrm{x}$ & $\mathrm{x}$ & $\mathrm{x}$ \\
\hline & Thsp1-18Ba-1 & Hypselodoris sp. a & 1 & 8 & 11.5 & & & & & & & & & & & & & & & & 1 & & & - & - & - \\
\hline & & $\begin{array}{c}\text { Mexichromis aurora } \\
\text { (Johnson and Gosliner, 1998) }\end{array}$ & 1 & 11 & 8.6 & & & & & & & & & & & 1 & & & & & & & & - & - & - \\
\hline & & $\begin{array}{c}\text { Mexichromis trilineata } \\
\text { (A. Adams and Reeve, 1850) * }\end{array}$ & $\mathrm{x}$ & & & & & & & $x$ & & & & & & & & & & & & & & - & - & - \\
\hline & Dorid18Ba-1 & Miamira magnifica & 1 & 2.5 & 20 & & & & & & & & & & & & & & 1 & & & & & $\mathrm{x}$ & - & - \\
\hline & Misp17Ba_1 & Miamira sp. a & 1 & 10 & 4 & & & & & & & & 1 & & & & & & & & & & & - & - & - \\
\hline & & Verconia simplex Pease, 1871 & 1 & 6 & 5.2 & & & & & 1 & & & & & & & & & & & & & & - & - & - \\
\hline & Thfu18Ba-1-2 & Thorunna furtiva Bergh, 1878 & 2 & $14-16$ & 13.8 & & & & & & & & & & & & & & 2 & & & & & $\mathrm{x}$ & - & - \\
\hline $\begin{array}{c}\text { Dendrodorididae } \\
\text { O'Donoghue, } \\
1924 \text { (1864) }\end{array}$ & & $\begin{array}{c}\text { Dendrodoris nigra (Stimpson, } \\
\text { 1855) }\end{array}$ & 1 & 6 & 1 & & & & & & & & 1 & & & & & & & & & & & - & - & - \\
\hline
\end{tabular}


Table 2. Cont.

\begin{tabular}{|c|c|c|c|c|c|c|c|c|c|c|c|c|c|c|c|c|c|c|c|c|c|c|c|c|c|c|}
\hline \multirow[b]{3}{*}{$\begin{array}{l}\text { Higher Taxon } \\
\text { Affiliation }\end{array}$} & \multicolumn{23}{|c|}{ Expedition in Bangka Archipelago and Mainland Nearby } & \multicolumn{3}{|c|}{$\begin{array}{c}\text { Other } \\
\text { Expeditions }\end{array}$} \\
\hline & \multirow[b]{2}{*}{ Identifier } & \multirow[b]{2}{*}{ Species Name } & \multirow[b]{2}{*}{ 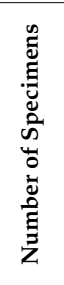 } & \multirow[b]{2}{*}{ 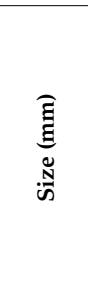 } & \multirow[b]{2}{*}{$\begin{array}{l}\widehat{\Xi} \\
\bar{\Xi} \\
\overline{0} \\
\text { ص̆ }\end{array}$} & \multicolumn{13}{|c|}{ Bangka Archipelago } & \multicolumn{5}{|c|}{ Mainland } & \multirow[b]{2}{*}{ 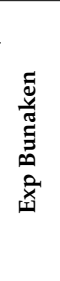 } & \multirow[b]{2}{*}{ 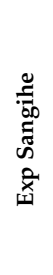 } & \multirow[b]{2}{*}{ 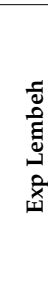 } \\
\hline & & & & & & 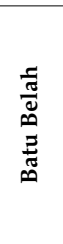 & 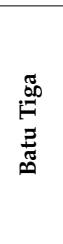 & 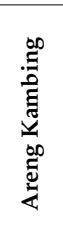 & 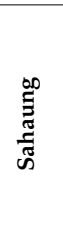 & 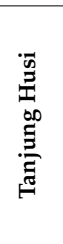 & 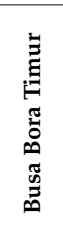 & 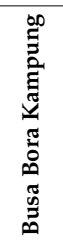 & 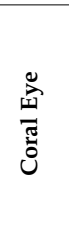 & 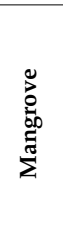 & $\overrightarrow{\tilde{s}}$ & 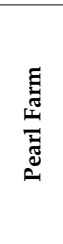 & 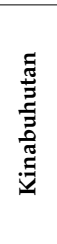 & $\begin{array}{l}\widetilde{\varpi} \\
\stackrel{\mathscr{\varpi}}{\widetilde{J}} \\
\widetilde{\pi}\end{array}$ & 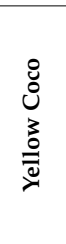 & $\begin{array}{l}\ddot{\Xi} \\
\text { : } \\
\text { ஸे }\end{array}$ & 苟 & 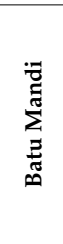 & 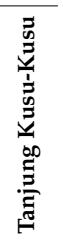 & & & \\
\hline \multirow{16}{*}{$\begin{array}{c}\text { Phyllidiidae } \\
\text { Rafinesque, } 1814\end{array}$} & & $\begin{array}{l}\text { Phyllidia cf. babai Brunckhorst, } \\
1993^{*}\end{array}$ & $\mathrm{x}$ & & & $\mathrm{x}$ & & & & & & & & & & & & & & & & & & - & - & - \\
\hline & & Phyllidia coelestis Bergh, 1905 & 8 & $12-43$ & $1.5-20$ & $x$ & & & 1 & & 1 & & 1 & & & & & 1 & 3 & 1 & & & & $\mathrm{x}$ & $\mathrm{x}$ & - \\
\hline & & Phyllidia elegans Bergh, 1869 & 3 & $34-38$ & 2 & & & & & & & & & & & & 1 & & 2 & & & & & $\mathrm{x}$ & - & $\mathrm{x}$ \\
\hline & & $\begin{array}{l}\text { Phyllidia exquisita Brunckhorst, } \\
1993\end{array}$ & 2 & $30-32$ & $4.5-12.4$ & & & & 1 & & & & 1 & & & & & & & & & & & - & - & - \\
\hline & & Phyllidia ocellata Cuvier, 1804 & 8 & $14-40$ & $2-13.4$ & 1 & & & & & 1 & & 2 & & & & & & 1 & 2 & 1 & & & $\mathrm{x}$ & $\mathrm{x}$ & - \\
\hline & & Phyllidia picta Pruvot-Fol, 1957 & 5 & $18-35$ & $4-12$ & 2 & & & & & 1 & & 1 & & & & & & & 1 & & & & - & $\mathrm{x}$ & - \\
\hline & & Phyllidia varicosa Lamarck, 1801 & 18 & $23-85$ & $2-20$ & & & 1 & & 5 & & & 1 & & 2 & & 1 & 5 & 1 & & 1 & & 1 & $\mathrm{x}$ & $\mathrm{x}$ & $\mathrm{x}$ \\
\hline & Phsp18Ba-2-3 & Phyllidia sp. a & 2 & $33-34$ & $6-13.5$ & & & & & & & & & & & & & & & 1 & 1 & & & - & $x$ & - \\
\hline & & $\begin{array}{l}\text { Phyllidiella annulata (Gray, } \\
\text { 1853) }\end{array}$ & 1 & 30 & 10 & & & & & & & & & & & & & 1 & & & & & & $\mathrm{x}$ & - & - \\
\hline & & $\begin{array}{l}\text { Phyllidiella lizae Brunckhorst, } \\
1993\end{array}$ & 4 & 15.26 & $5-20$ & & & & & & & & & & & & & 1 & 1 & 2 & & & & - & - & - \\
\hline & & $\begin{array}{c}\text { Phyllidiella nigra (van Hasselt } \\
\text { 1824) }\end{array}$ & 1 & 56 & 1 & & & & & & & & & 1 & & & & & & & & & & - & $\mathrm{x}$ & - \\
\hline & & $\begin{array}{c}\text { Phyllidiella pustulosa (Cuvier, } \\
\text { 1804) }\end{array}$ & 39 & $12-75$ & $1.5-20$ & 5 & & 2 & 2 & 1 & & 2 & 7 & & & 1 & 3 & 3 & 2 & 2 & 6 & 1 & 2 & $\mathrm{x}$ & $x$ & $x$ \\
\hline & & $\begin{array}{l}\text { Phyllidiopsis annae Brunckhorst, } \\
1993\end{array}$ & 4 & 3-12 & $9.2-11.9$ & & & & & & & 2 & & & & & & & & 2 & & & & - & - & - \\
\hline & & $\begin{array}{l}\text { Phyllidiopsis cf. burni } \\
\text { Brunckhorst, } 1993\end{array}$ & 1 & 39 & 20 & & & & & & & & & & & & & & 1 & & & & & - & - & - \\
\hline & & $\begin{array}{l}\text { Phyllidiopsis krempfi Pruvot-Fol, } \\
1957\end{array}$ & 3 & $17-56$ & $9.2-16.2$ & & & 1 & & & & & & & & & & & & 1 & & 1 & & - & $x$ & - \\
\hline & & $\begin{array}{c}\text { Phyllidiopsis xishaensis } \\
\text { (Lin,1983) }\end{array}$ & 4 & $9-17$ & $3.9-16.8$ & & & & & 1 & & & & & & 2 & & & & & & 1 & & $\mathrm{x}$ & - & - \\
\hline
\end{tabular}


Table 2. Cont

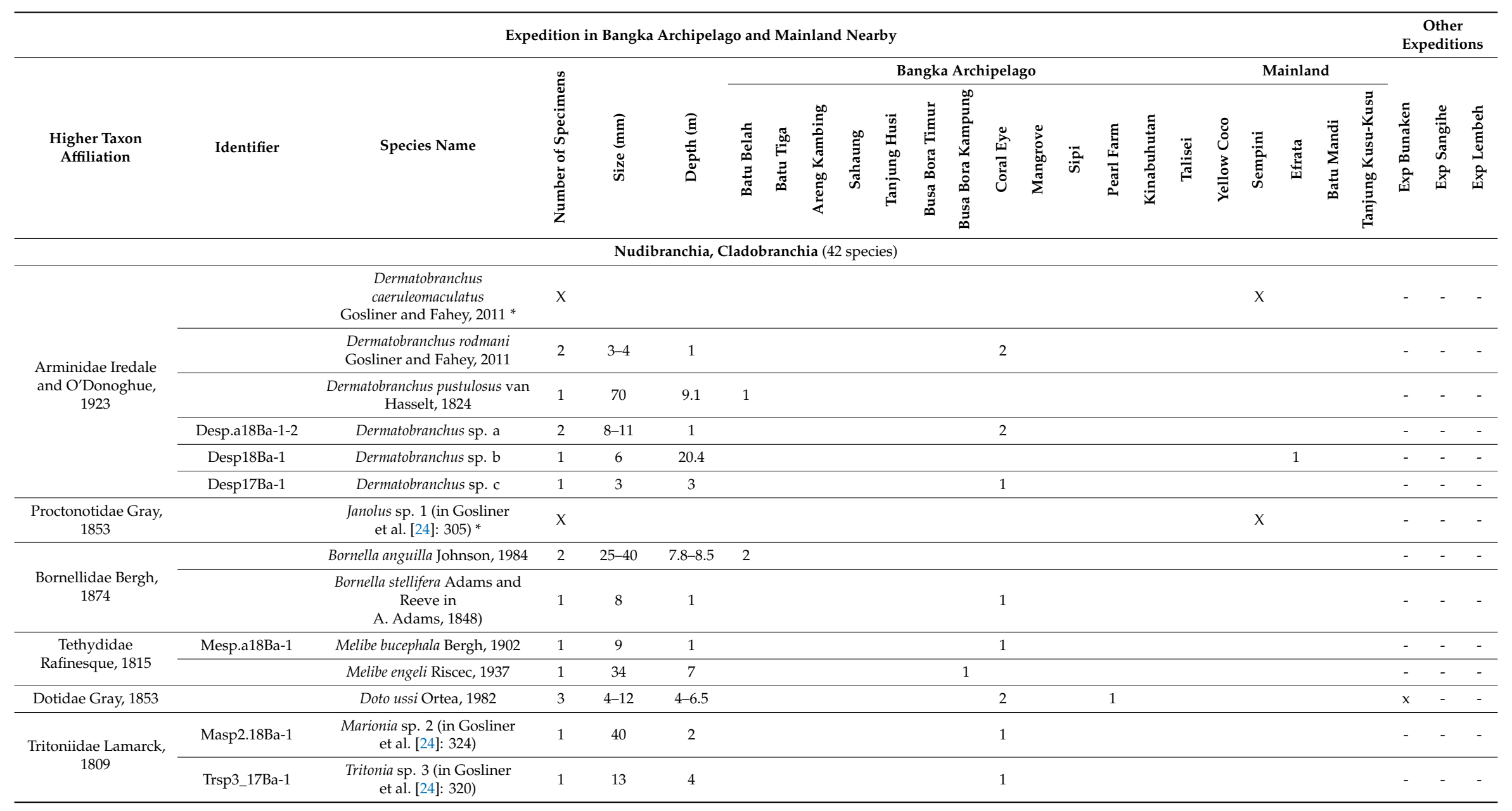


Table 2. Cont.

\begin{tabular}{|c|c|c|c|c|c|c|c|c|c|c|c|c|c|c|c|c|c|c|c|c|c|c|c|c|c|c|}
\hline \multirow[b]{3}{*}{$\begin{array}{l}\text { Higher Taxon } \\
\text { Affiliation }\end{array}$} & \multicolumn{23}{|c|}{ Expedition in Bangka Archipelago and Mainland Nearby } & \multicolumn{3}{|c|}{$\begin{array}{c}\text { Other } \\
\text { Expeditions }\end{array}$} \\
\hline & \multirow[b]{2}{*}{ Identifier } & \multirow[b]{2}{*}{ Species Name } & \multirow[b]{2}{*}{ 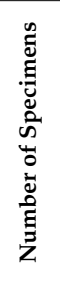 } & \multirow[b]{2}{*}{ 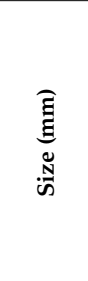 } & \multirow[b]{2}{*}{ 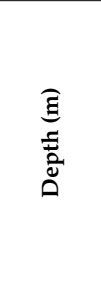 } & \multicolumn{12}{|c|}{ Bangka Archipelago } & \multicolumn{6}{|c|}{ Mainland } & \multirow[b]{2}{*}{ 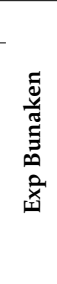 } & \multirow[b]{2}{*}{ 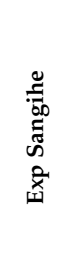 } & \multirow[b]{2}{*}{ 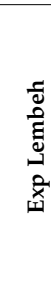 } \\
\hline & & & & & & 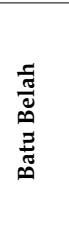 & 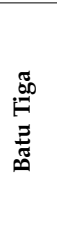 & 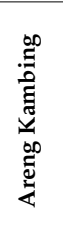 & 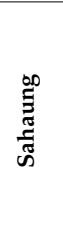 & 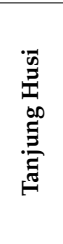 & 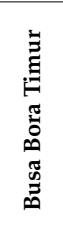 & 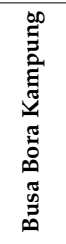 & $\frac{\text { 兽 }}{\text { 嵒 }}$ & 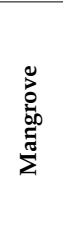 & $\overrightarrow{\mathrm{n}}$ & 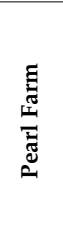 & 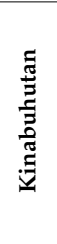 & 离 & $\begin{array}{l}8 \\
0 \\
3 \\
0 \\
\bar{\Xi}\end{array}$ & 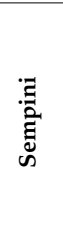 & 菢 & 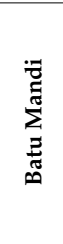 & 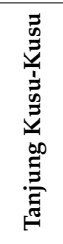 & & & \\
\hline \multirow{4}{*}{$\begin{array}{l}\text { Flabellinidae Bergh, } \\
1889\end{array}$} & & $\begin{array}{c}\text { Coryphellina exoptata } \\
\text { (Gosliner and Willan, 1991) } \\
\end{array}$ & 3 & $16-32$ & $2-12.4$ & 1 & & & & & & 1 & & & & 1 & & & & & & & & $\mathrm{x}$ & - & - \\
\hline & & $\begin{array}{l}\text { Coryphellina rubrolineata } \\
\text { O'Donoghue, } 1929\end{array}$ & 8 & $20-40$ & $6.3-20.4$ & 1 & & 2 & & & & & & & & 2 & & & & 1 & 1 & & 1 & $\mathrm{x}$ & - & $\mathrm{x}$ \\
\hline & Flsp2-18Ba-1 & $\begin{array}{l}\text { Flabellina sp. } 2 \text { (in Gosliner } \\
\text { et al. [24]: } 333 \text { ) }\end{array}$ & 1 & 16 & 14.5 & & & & & & & & & & 1 & & & & & & & & & - & - & - \\
\hline & Flsp3_17Ba-1 & $\begin{array}{l}\text { Flabellina sp. 3 (in Gosliner } \\
\text { et al. [24]: 333) }\end{array}$ & 1 & 11 & 13.6 & & & & & & & & & & & & & & & & & 1 & & - & - & - \\
\hline $\begin{array}{c}\text { Samlidae } \\
\text { Korshunova, } \\
\text { Martynov, Bakken, } \\
\text { Evertsen, Fletcher, } \\
\text { Mudianta, Saito, } \\
\text { Lundin, Schrödl and } \\
\text { Picton, 2017 }\end{array}$ & & $\begin{array}{l}\text { Samla riwo (Gosliner and } \\
\text { Willan, 1991) }\end{array}$ & 3 & $9-13$ & $4-8.6$ & & & & & & & & 2 & & & & & & & & & 1 & & $x$ & - & - \\
\hline $\begin{array}{l}\text { Eubranchidae } \\
\text { Odhner, } 1934\end{array}$ & & $\begin{array}{l}\text { Eubranchus sp. } 22 \text { (in Gosliner } \\
\text { et al. [24]: } 341 \text { ) }\end{array}$ & 1 & 6 & 5.1 & & & 1 & & & & & & & & & & & & & & & & $x$ & - & - \\
\hline $\begin{array}{l}\text { Trinchesiidae F. } \\
\text { Nordsieck, } 1972\end{array}$ & & $\begin{array}{l}\text { Trinchesia yamasui (Hamatani, } \\
\text { 1993) }\end{array}$ & $x$ & & & & & & & & & & & & & & & & & $x$ & & & & - & - & - \\
\hline $\begin{array}{l}\text { Cuthonidae Odhner, } \\
1934\end{array}$ & & $\begin{array}{l}\text { Cuthona sp. } 57 \text { (in Gosliner } \\
\text { et al. [24]: } 353 \text { ) }\end{array}$ & 2 & $17-25$ & 12.7 & & & & & & & & 2 & & & & & & & & & & & - & - & - \\
\hline
\end{tabular}


Table 2. Cont.

\begin{tabular}{|c|c|c|c|c|c|c|c|c|c|c|c|c|c|c|c|c|c|c|c|c|c|c|c|c|c|c|}
\hline \multirow[b]{3}{*}{$\begin{array}{l}\text { Higher Taxon } \\
\text { Affiliation }\end{array}$} & \multirow[b]{3}{*}{ Identifier } & \multicolumn{22}{|c|}{ Expedition in Bangka Archipelago and Mainland Nearby } & \multicolumn{3}{|c|}{$\begin{array}{c}\text { Other } \\
\text { Expeditions }\end{array}$} \\
\hline & & \multirow{2}{*}{\multicolumn{2}{|c|}{ 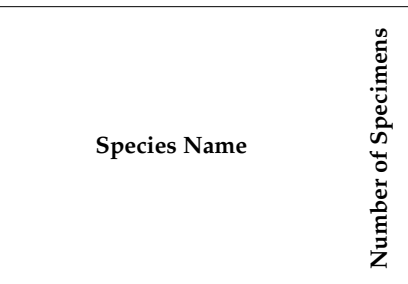 }} & \multirow[b]{2}{*}{$\begin{array}{l}\widehat{\Xi} \\
\text { हू } \\
\stackrel{\tilde{N}}{\omega}\end{array}$} & \multirow[b]{2}{*}{$\begin{array}{l}\widehat{\bar{\Xi}} \\
\overline{\bar{a}} \\
\text { ڤ̆ }\end{array}$} & \multicolumn{13}{|c|}{ Bangka Archipelago } & \multicolumn{5}{|c|}{ Mainland } & \multirow[b]{2}{*}{ 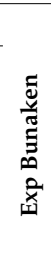 } & \multirow[b]{2}{*}{ 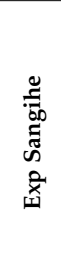 } & \multirow[b]{2}{*}{ 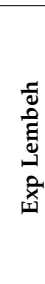 } \\
\hline & & & & & & 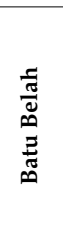 & $\begin{array}{l}\stackrel{\infty}{E} \\
\stackrel{D}{E} \\
\stackrel{D}{E}\end{array}$ & 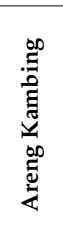 & 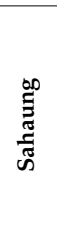 & 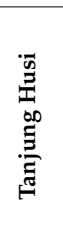 & 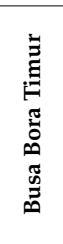 & 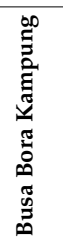 & 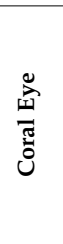 & 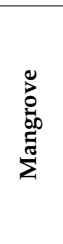 & $\ddot{\bar{n}}$ & 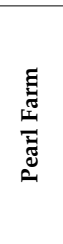 & 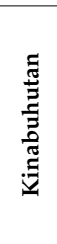 & $\begin{array}{l}\widetilde{\varpi} \\
\stackrel{.}{\widetilde{\sigma}} \\
\widetilde{\sim}\end{array}$ & $\begin{array}{l}8 \\
0 \\
3 \\
0 \\
\bar{\Xi}\end{array}$ & $\begin{array}{l}\vec{a} \\
\text { à } \\
\text { के }\end{array}$ & 苟 & 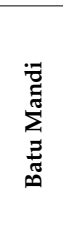 & 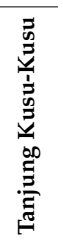 & & & \\
\hline \multirow{15}{*}{$\begin{array}{l}\text { Facelinidae Bergh, } \\
1889\end{array}$} & Aeol18Ba-1 & Aeolidia sp. a & 1 & 8 & 1.5 & & & & & & & & 1 & & & & & & & & & & & - & - & - \\
\hline & Ansp17Ba-1 & Antonietta sp. a & 1 & 2 & 6.4 & & & & & & & & & & & & & & & & & 1 & & - & - & - \\
\hline & & Caloria indica (Bergh, 1896) & 15 & $2-38$ & $5-13.4$ & 5 & & & & 2 & & & 3 & & & 1 & & & & 1 & 2 & 1 & & $\mathrm{x}$ & - & - \\
\hline & Crsp17Ba-1 & Cratena sp. a & 1 & 8 & 4 & & & & & & & & 1 & & & & & & & & & & & - & - & - \\
\hline & & Favorinus japonicus Baba, 1949 & 2 & $10-16$ & $8.9-20$ & & & & & & & & & & & 1 & & & 1 & & & & & $x$ & - & - \\
\hline & Fasp1-18Ba-1-2 & $\begin{array}{l}\text { Favorinus sp. } 1 \text { (in Gosliner } \\
\text { et al. [24]: 363) }\end{array}$ & 2 & $13-15$ & 20 & & & & & & & & & & & & & & & 2 & & & & - & - & - \\
\hline & & $\begin{array}{l}\text { Favorinus tsuruganus Baba and } \\
\text { Abe, } 1964\end{array}$ & 1 & 11 & 2 & & & & & & & & 1 & & & & & & & & & & & - & - & - \\
\hline & Mojo18Ba-1 & Moridilla sp. a & 2 & 12 & $6.8-11.2$ & 1 & & & & 1 & & & & & & & & & & & & & & - & - & - \\
\hline & & $\begin{array}{l}\text { Noumeaella sp. } 2 \text { (in Gosliner } \\
\text { et al. [24]: } 367 \text { ) }\end{array}$ & 1 & 11 & 4 & & & & & & & & 1 & & & & & & & & & & & - & - & - \\
\hline & & $\begin{array}{l}\text { Noumeaella sp. } 3 \text { (in Gosliner } \\
\text { et al. [24]: } 367 \text { ) }\end{array}$ & 6 & $8-12$ & $4-9$ & & & & & & & & 6 & & & & & & & & & & & $\mathrm{x}$ & - & - \\
\hline & & $\begin{array}{l}\text { Noumeaella sp. } 13 \text { (in Gosliner } \\
\text { et al. [24]: 369) }\end{array}$ & 7 & $7-16$ & 3-9 & & & & & & & & 7 & & & & & & & & & & & - & - & - \\
\hline & & $\begin{array}{l}\text { Phyllodesmium briareum (Bergh, } \\
1896 \text { ) }\end{array}$ & 17 & $15-60$ & $3-20$ & & & & & & & & 16 & & & & & & 1 & & & & & $x$ & - & - \\
\hline & & $\begin{array}{l}\text { Phyllodesmium cf. crypticum } \\
\text { Rudman, } 1981\end{array}$ & 5 & $8-22$ & 4 & & & & & & & & 5 & & & & & & & & & & & - & - & - \\
\hline & & $\begin{array}{c}\text { Phyllodesmium lizardense } \\
\text { Burghardt, Schrödl and } \\
\text { Wägele, } 2008\end{array}$ & 3 & $10-13$ & $1-10$ & & & & & & & 1 & 2 & & & & & & & & & & & - & - & - \\
\hline & & $\begin{array}{l}\text { Phyllodesmium longicirrum } \\
\text { (Bergh, 1905) }\end{array}$ & 1 & 125 & 25 & & & & & & & & & & & & & & & & 1 & & & - & - & - \\
\hline
\end{tabular}


Table 2. Cont.

\begin{tabular}{|c|c|c|c|c|c|c|c|c|c|c|c|c|c|c|c|c|c|c|c|c|c|c|c|c|c|c|}
\hline \multirow[b]{3}{*}{$\begin{array}{l}\text { Higher Taxon } \\
\text { Affiliation }\end{array}$} & \multirow[b]{3}{*}{ Identifier } & \multicolumn{22}{|c|}{ Expedition in Bangka Archipelago and Mainland Nearby } & \multicolumn{3}{|c|}{$\begin{array}{c}\text { Other } \\
\text { Expeditions }\end{array}$} \\
\hline & & \multirow{2}{*}{\multicolumn{2}{|c|}{ 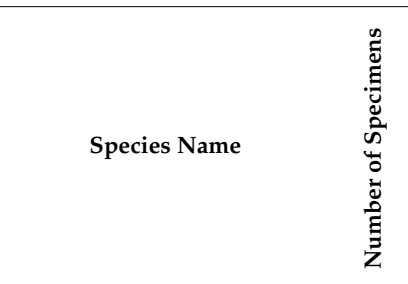 }} & \multirow[b]{2}{*}{$\begin{array}{l}\bar{\Xi} \\
\text { ह्र } \\
\stackrel{\Xi}{N} \\
\text { के }\end{array}$} & \multirow[b]{2}{*}{ 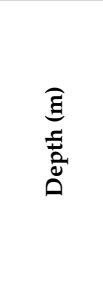 } & \multicolumn{12}{|c|}{ Bangka Archipelago } & \multicolumn{6}{|c|}{ Mainland } & \multirow[b]{2}{*}{ 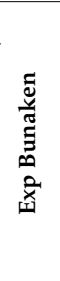 } & \multirow[b]{2}{*}{ 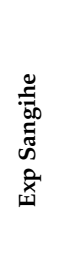 } & \multirow[b]{2}{*}{ 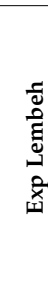 } \\
\hline & & & & & & 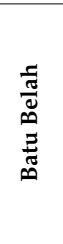 & 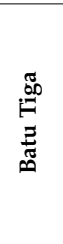 & 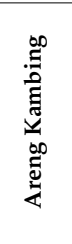 & $\begin{array}{l}\infty \\
\Xi_{\pi}^{\infty} \\
\frac{\pi}{\pi} \\
\omega\end{array}$ & 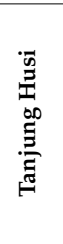 & 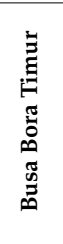 & 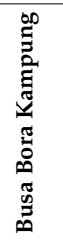 & 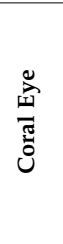 & 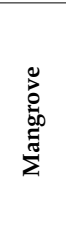 & $\ddot{n}$ & 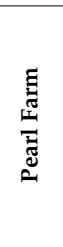 & 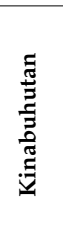 & 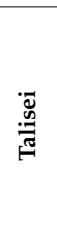 & $\begin{array}{l}8 \\
0 \\
z \\
0 \\
\bar{\Xi}\end{array}$ & 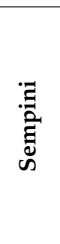 & 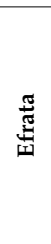 & 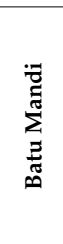 & 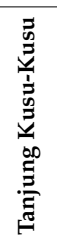 & & & \\
\hline & & $\begin{array}{l}\text { Phyllodesmium magnum } \\
\text { Rudman, } 1991\end{array}$ & 2 & 50 & $5.5-11.5$ & & & & & 1 & & & 1 & & & & & & & & & & & - & - & - \\
\hline & & $\begin{array}{l}\text { Phyllodesmium parangatum } \\
\text { Ortiz and Gosliner, } 2003\end{array}$ & 5 & $8-15$ & $1-7$ & & & & & & & & 5 & & & & & & & & & & & - & - & - \\
\hline & & $\begin{array}{l}\text { Phyllodesmium pecten Rudman, } \\
1981\end{array}$ & 1 & 15 & 1 & & & & & & & & 1 & & & & & & & & & & & - & - & - \\
\hline & & $\begin{array}{l}\text { Phyllodesmium poindimiei } \\
\text { (Risbec, 1928) }\end{array}$ & 2 & $14-21$ & $4-8.9$ & & & & & & & & 1 & & & & & & & & 1 & & & $\mathrm{x}$ & - & - \\
\hline & & $\begin{array}{c}\text { Pteraeolidia semperi (Bergh, } \\
1870 \text { ) }\end{array}$ & 25 & $13-54$ & $5-8.9$ & 2 & & 2 & & 1 & & & & & & & & & & 15 & 2 & 3 & & $\mathrm{x}$ & - & - \\
\hline \multicolumn{27}{|c|}{ Eupulmonata (1 species) } \\
\hline & Onchidiidae & Peronia sp. a & 1 & 39 & 1 & & & & & & & & & 1 & & & & & & & & & & - & - & - \\
\hline & Total & & 149 & & & 34 & 0 & 11 & 14 & 40 & 14 & 23 & 162 & 8 & 13 & 16 & 7 & 12 & 23 & 41 & 30 & 23 & 14 & - & - & - \\
\hline
\end{tabular}



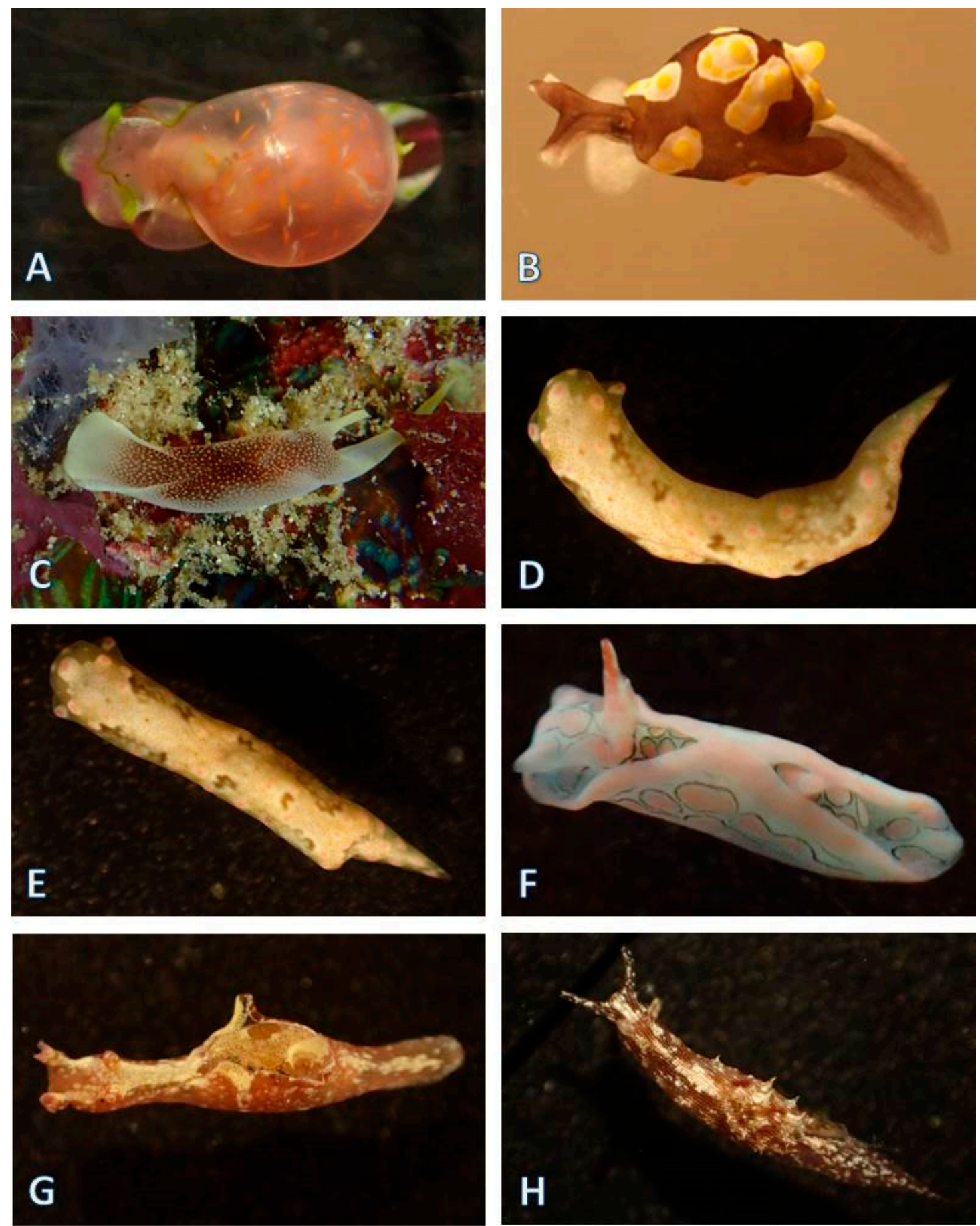

Figure 2. Cephalaspidea and Aplysiida: (A) Haminoea sp. (Haminoea sp. 3 in Gosliner et al. [24]: 34), Hasp18Ba-1; (B) Colpodaspis thompsoni, Coth18Ba-2; (C) Chelidonura amoena, Cham17Ba-1; (D,E) Odontoglaja sp. (Odontoglaja sp. a), Odsp.a18Ba-1; (F) Sagaminopteron psychedelicum, Saps18Ba-2;

(G) Aplysia cf. nigrocincta, Appa18Ba-1; (H) Stylocheilus striatus, Stst18Ba-2.

\subsubsection{Aplysiida (= Anaspidea) (Two Species in Two Genera Belonging to One Family)}

Aplysiidae: Members of this family mainly forage on algae in shallow water. Four specimens of probably Aplysia cf. nigrocincta (Figure 2G) (Appa18Ba-3, with a black line along the parapodia) were found under coral rubble. Recently, Golestani et al. [29] revised Aplysia parvula and identified 10 different lineages within this species. They resurrected the name A. nigrocincta Martens, 1880 for 
specimens from the Philippines and Papua New Guinea. However, molecular analyses still need to be performed on our material for correct assignment. The same holds true for the specimens described in Eisenbarth et al. [16] as A. parvula. Two specimens of Stylocheilus striatus (Figure 2H) were found under coral rubble.

\subsubsection{Sacoglossa (15 Species in Four Genera Belonging to Three Families)}

Oxynoideae: Lobiger sp. 1 (Figure 3A), mimicking the green alga Caulerpa, has elongate leaf-like cerata and a green bubble-like shell. Our two specimens lack the blue lines typical for L. viridis (as depicted in Gosliner et al. [24]) and seem to be undescribed.

Hermaeidae: According to Bouchet et al. [30], the family Caliphyllidae is united with this family, and we follow their systematics. Two Cyerce species were collected: one specimen of $C$. bourbonica (Figure 3B) shows the characteristic opaque white body, dark brown marks along the cerata and rhinophores, and violet to pink color at the tip of cerata. The other species can be assigned to $C$. nigra (Figure 3C), based on the typical color and color pattern of the cerata.

Plakobranchidae: Only the genera Elysia and Thuridilla are present in our collections. Elysia asbecki (Figure 3D), E. marginata (Figure 3E), E. cf. nigropunctata (Figure 3F), E. pusilla (Figure 3G), and four unidentified Elysia species were sampled from various algae in the coral rubble or highly structured micro-habitats in the coral reef. E. cf. nigropunctata (Elni18Ba-1) was collected from a sponge, probably feeding on its epiphytic green algae. Four undescribed Elysia spp. (Elysia sp. 23, Elysia sp. 27, Elysia sp. a, and Elysia sp. b) in our collection indicate the need for further investigation with regard to this group: Elysia sp. 24 (Elsp24-18Ba-1; Figure 3H) and Elysia sp. 27 (Elsp27-18Ba-1; Figure 4A) are depicted in Gosliner et al. [24], whereas Elysia sp. a (Elsp.a18Ba-1; Figure 4B) and Elysia sp. b (Elsp.b18Ba-1; Figure $4 \mathrm{C}$ ) are featured here for the first time. These species were not found previously in North Sulawesi. Elysia sp. 24 has numerous white dots on its body, the rhinophores have three white bands, and the light green parapodia are without a line at the edge. Elysia sp. 27 (Elsp27-18Ba-1), collected from Halimeda, is pale green with brown dots and numerous opaque white spots on the dorsum and rhinophores; an orange line runs along the edge of the parapodia. Elysia sp. a (Figure 4B) looks like Elysia sp. 24 [24] but has a red line along the mantle margin. Elysia sp. b (Figure 4C) has an opaque white body color with small green dots covering the dorsal notum and parapodia. The rhinophores are relatively short, with papillae, and a brown line running along the margin of the parapodia crosses the foot on the ventral side. Four Thuridilla species were collected, including T. carlsoni (Figure 4D), T. flavomaculata (Figure 4E), T. vataae (Figure 4F), and T. cf. gracilis (Figure 4G,H), the latter being the most common Thuridilla species in the study area. According to Martín-Hervás et al. [31], this species consists of a complex of 14 species. We cannot assign our specimens to any of these cryptic species, but we can provide more details on the coloration especially of the rhinophores that might help in future assignment of our material. Our specimens from Bangka have a black to dark brown background with narrow white to light green longitudinal lines, which are broader in certain areas, implicating transversal white interrupted patches. The parapodial edge exhibits a narrow orange line, but no blue spots on the outside of the parapodia. Some specimens have white rhinophores with a black tip apical to an orange band, and others have rhinophores only with orange tips (Figure 5A-E).

\subsubsection{Pleurobranchida (Two Species in One Genus Belonging to One Family)}

Pleurobranchida (Pleurobranchomorpha) are carnivorous, nocturnal animals usually large in size. We mainly observed the specimens during night dives.

Pleurobranchidae: Pleurobranchus forskalii $(>200 \mathrm{~mm}$ ) was only found during the night. The tubercles of this species (Figure 6A) are compound and brownish in color. The specimens of P. peronii (Figure 6B), also only found during night dives, have only simple tubercles. 

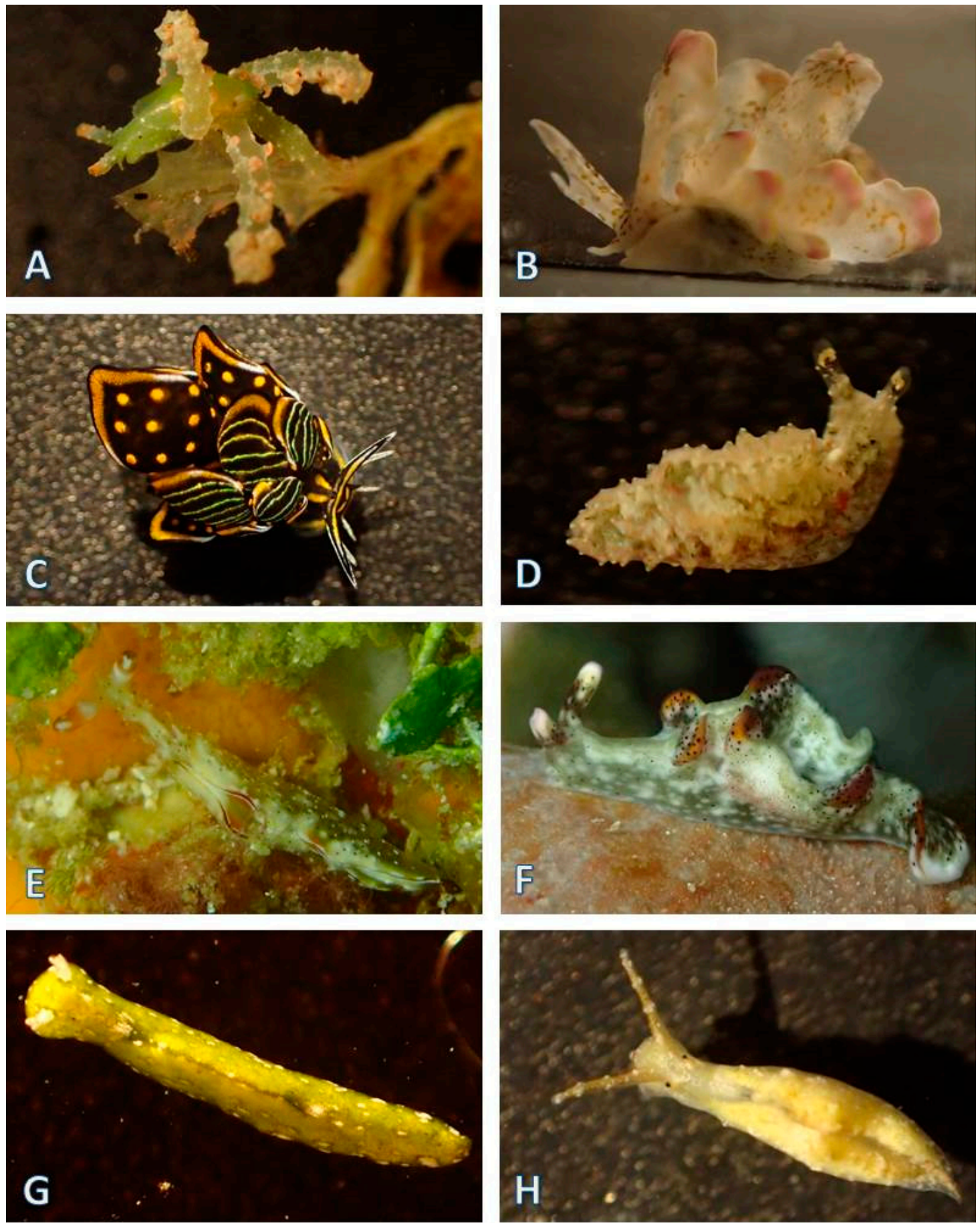

Figure 3. Sacoglossa: (A) Lobiger sp. (Lobiger sp. 1 in Gosliner et al. [24]: 70), Losp1-18Ba1; (B) Cyerce bourbonica, Cybo18Ba-1; (C) Cyerce nigra, Cyni18Ba-1; (D) Elysia asbecki, Elas18Ba-1; (E) Elysia marginata, Elma17Ba-1; (F) Elysia cf. nigropunctata, Elni18Ba-1;(G) Elysia pusilla, Elpu18Ba-2; (H) Elysia sp. (Elysia sp. 24 in Gosliner et al. [24]: 89), Elsp24-18Ba-1. 

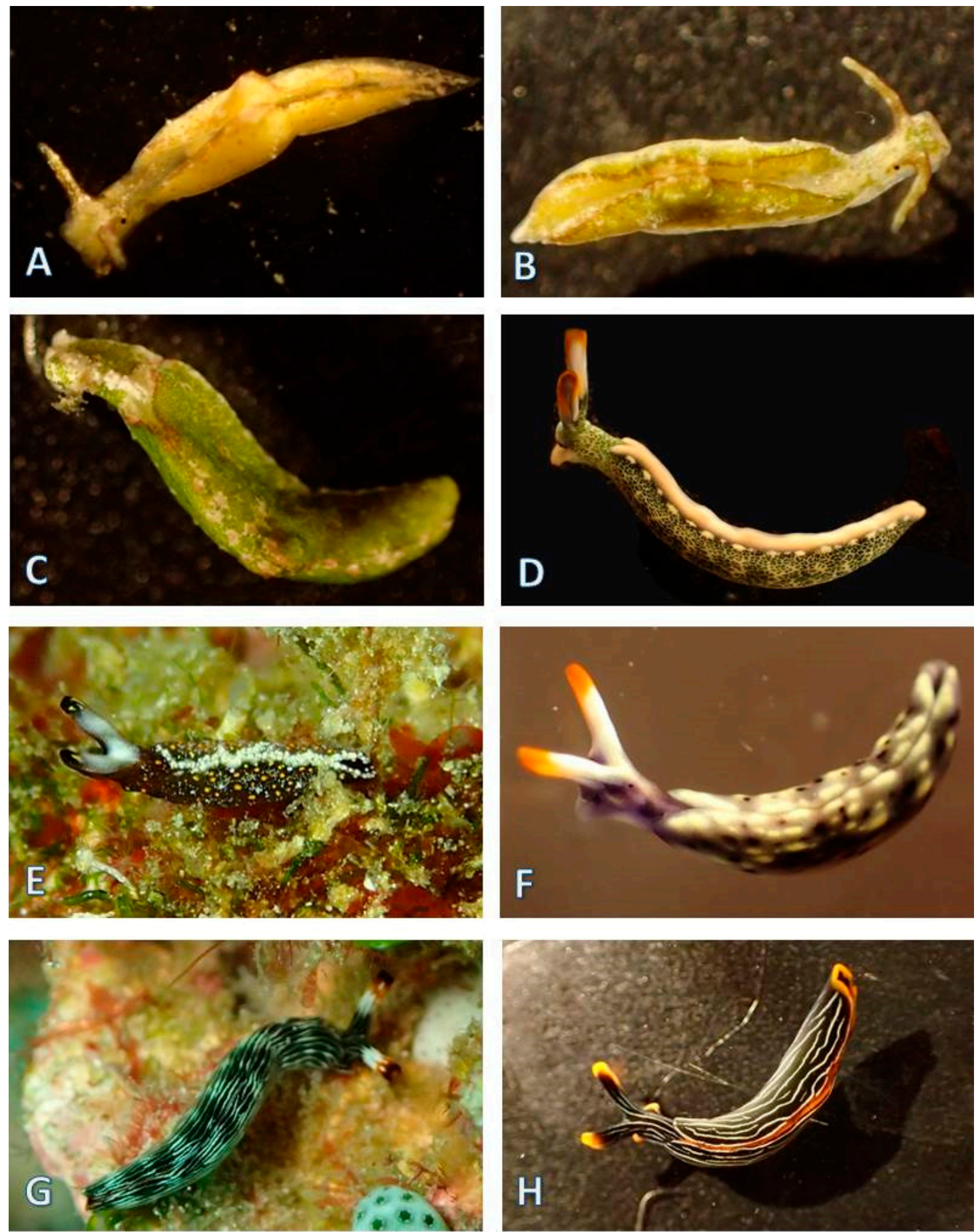

Figure 4. Sacoglossa: (A) Elysia sp. (Elysia sp. 27 in Gosliner et al. [24]: 89), Elsp27-18Ba-1; (B) Elysia sp. (Elysia sp. a), Elsp.a18Ba-1; (C) Elysia sp. (Elysia sp. b), Elsp.b18Ba-1; (D) Thuridilla carlsoni, Thca18Ba-1; (E) Thuridilla flavomaculata, Thfl17Ba-1; (F) Thuridilla vataae, Thva18Ba-3; (G,H) Thuridilla gracilis, Thgr18Ba-1, Thgr18Ba-4. 

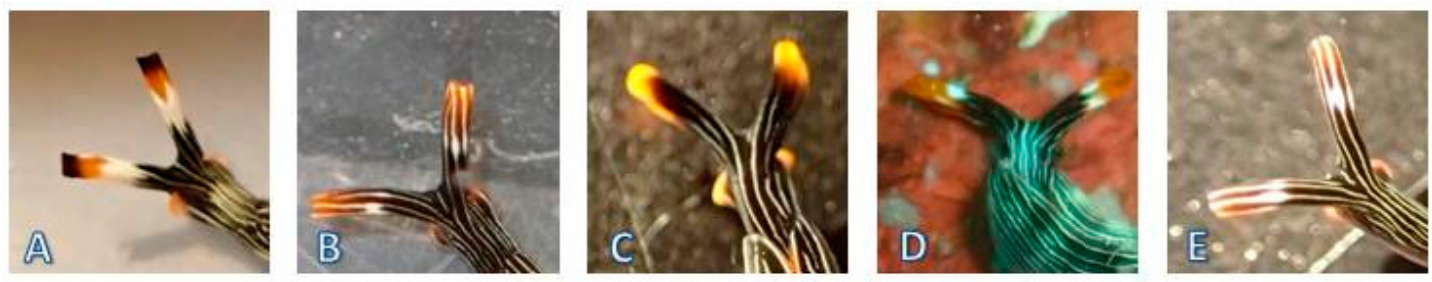

Figure 5. Variability of rhinophores in the Thuridilla gracilis species complex from Bangka Archipelago: (A) Thgr18Ba1; (B) Thgr17Ba-3; (C) Thgr18Ba4; (D) Thgr18Ba6; (E) Thgr18Ba-5.
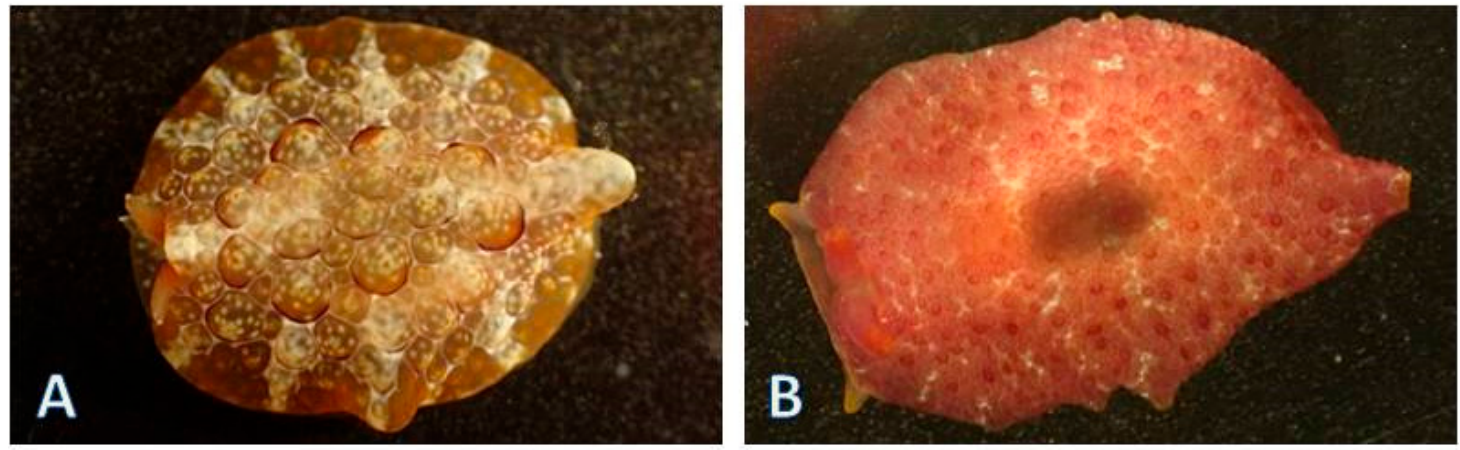

Figure 6. Pleurobranchida: (A) Pleurobranchus forskalii, Plfo18Ba-1; (B) Pleurobranchus peronii, Plpe18Ba-1.

\subsubsection{Nudibranchia (124 Species)}

a. Nudibranchia, Doridina (83 species in 29 genera belonging to eight families)

Doridina are represented in our study by nine families of the 17 listed in WoRMS (World Register of Marine Species) [26].

Hexabranchidae: One specimen of Hexabranchus sanguineus (Figure 7A) was found in coral rubble. It was a juvenile mimicking a Hypselodoris maculosa in coloration. Molecular analyses confirmed its assignment to $H$. sanguineus.

Polyceridae: The family is represented by 13 species in three genera. Compared to BNP and Sangihe, the genus Nembrotha is relatively common in Bangka Archipelago, with seven species records. Five specimens of $N$. chamberlaini (Figure 7B) and five specimens of N. cristata (Figure 7C) were collected at different sites and depths. N. kubaryana (Figure 7D), encountered quite often, is represented in our samples by 13 specimens, collected at 10 different sites. It exhibits some variation with regard to the presence of orange patterns or lines along the body. Two mating N. lineolata (Figure 7E) were only recorded by photographs. N. milleri (Figure 7F) was crawling on brown sponges near green tunicates. Two specimens of N. mullineri (Figure 7G) were collected only during two night dives, one in 2017 and one in 2018. In both cases, the animals were crawling on sand. One undescribed Nembrotha (Nesp1_17Ba-1; Figure 7H), similar to Nembrotha sp. 1 [24], was collected on a greenish tunicate; however, our specimen has bluish rhinophores and gills. Tambja gabrielae (Figure 8A) and T. morosa (Figure $8 \mathrm{~B}$ ) were collected at 8-17 $\mathrm{m}$ depth. The monotypic genus Gymnodoris within the Gymnodorididae comprises many similar species with at least 60 undescribed species [24]. The single specimen of G. aurita (Figure 8C) was identified only by photo-documentation. Gymnodoris tuberculosa (Figure 8D; $3 \mathrm{~mm}$ long) was crawling in coral rubble at $1 \mathrm{~m}$ depth. Two specimens of two Gymnodoris spp. (Figure 8E,F) are undescribed and depicted in Gosliner et al. [24] as Gymnodoris sp. 20 and Gymnodoris sp. 25, respectively. 

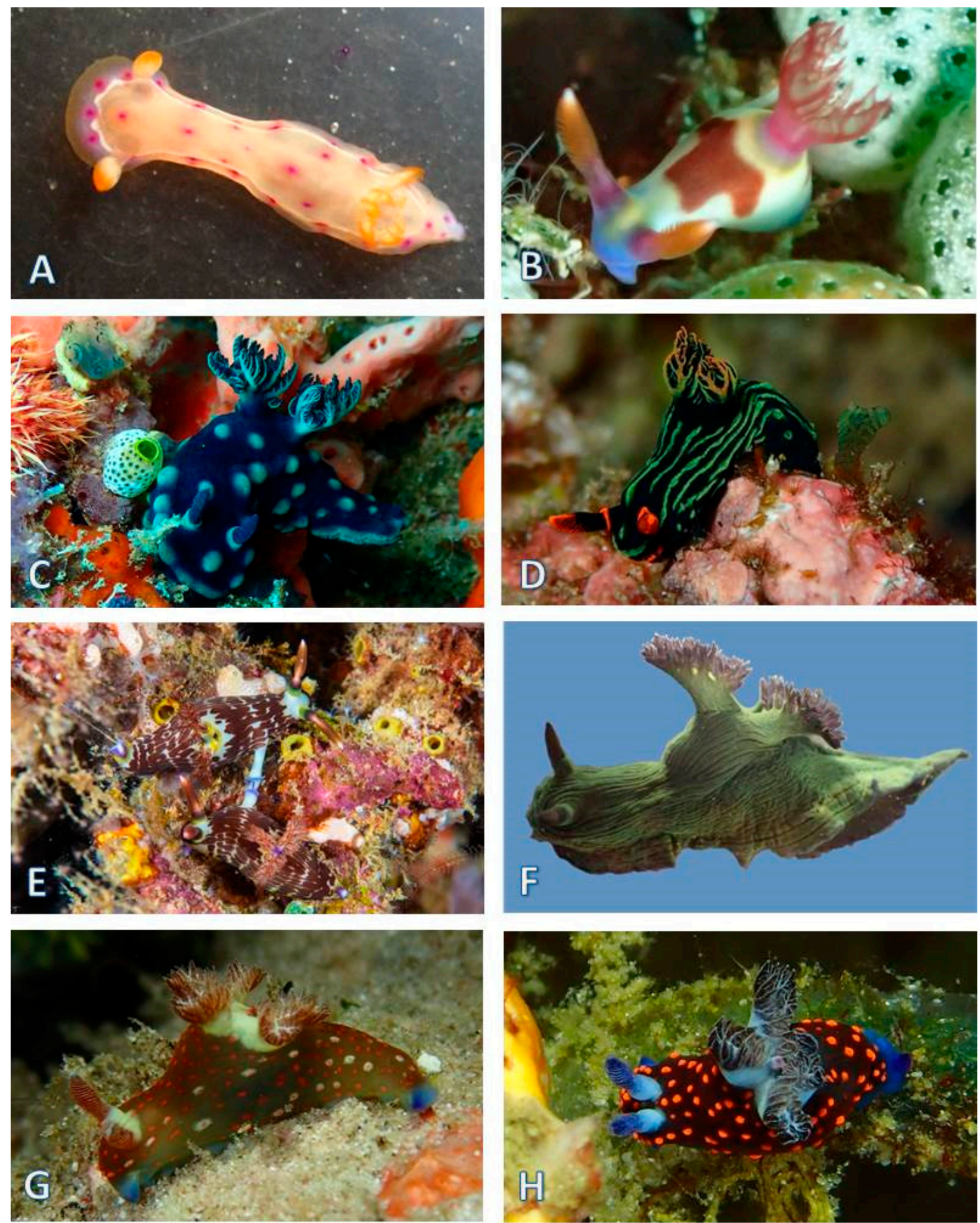

Figure 7. Nudibranchia Doridina: (A) Hexabranchus sanguineus, Glsp1_17Ba-1; (B) Nembrotha chamberlaini, Nech18Ba-1; (C) Nembrotha cristata Necr17Ba-2; (D) Nembrotha kubaryana, Neku17Ba-4; (E) Nembrotha lineolata*; (F) Nembrotha milleri, Nemi17Ba-1; (G) Nembrotha mullineri, Nemu17Ba-1; (H) Nembrotha sp. (Nembrotha sp. 1 in Gosliner et al. [24]: 122), Nesp1_17Ba-1. *Specimen not collected. 

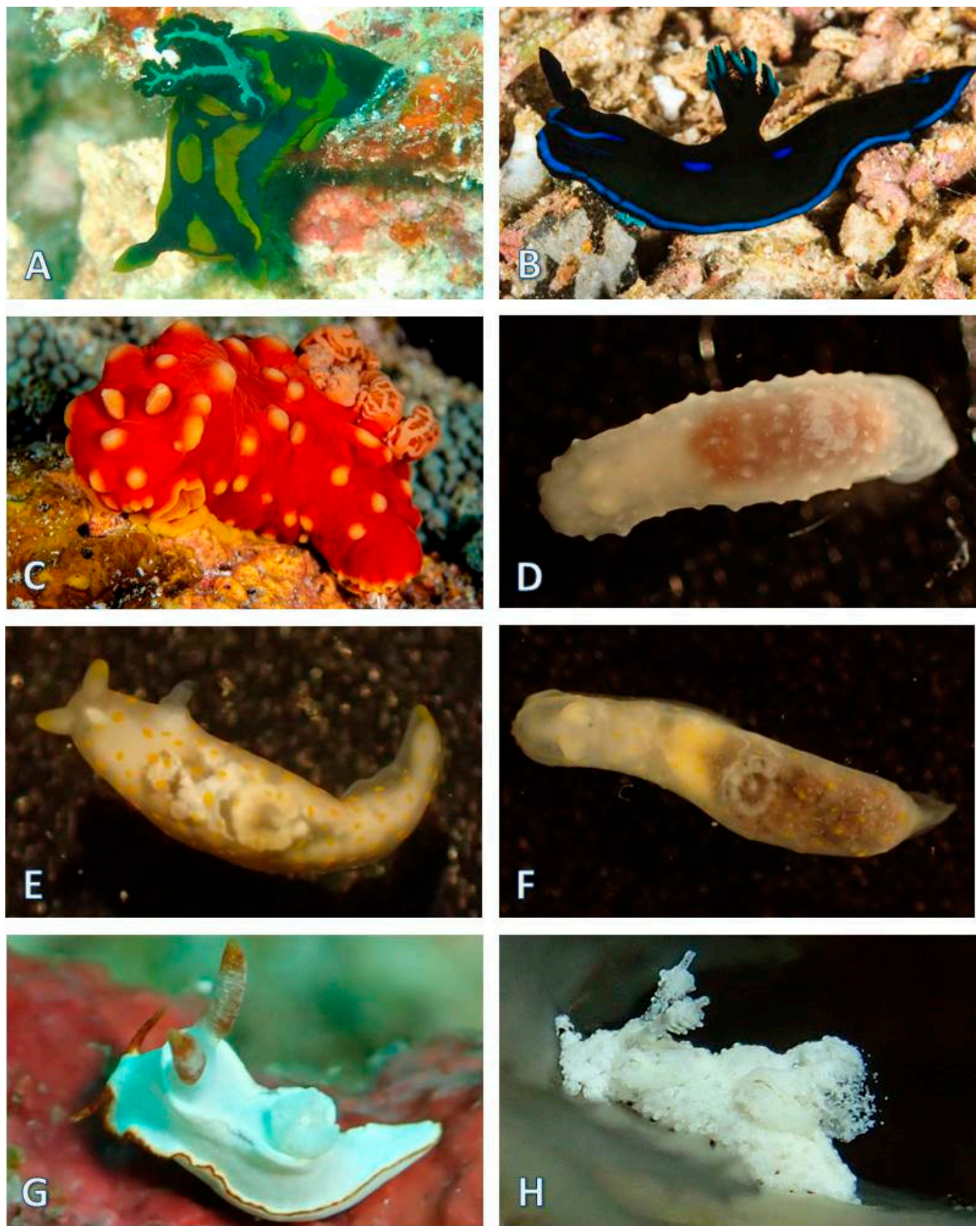

Figure 8. Nudibranchia Doridina: (A) Tambja gabrielae, Taga18Ba-1; (B) Tambja morosa*; (C) Gymnodoris aurita*; (D) Gymnodoris tuberculosa, Gytu18Ba-1; (E) Gymnodoris sp. (Gymnodoris sp. 20 in Gosliner et al. [24]: 156), Gysp20-18Ba1; (F) Gymnodoris sp. (Gymnodoris sp. 25 in Gosliner et al. [24]: 157), Gysp25-18Ba1; (G) Goniodoris sp. (Goniodoris sp. 7 in Gosliner et al. [24]: 153), Gosp7-18Ba1; (H) Trapania armilla, Trar17Ba-1. *Specimen not collected.

Goniodorididae: Members of this family are quite rare and not represented by many species in North Sulawesi [16]. Some undescribed Goniodoris were recorded by Gosliner et al. [24]. We collected only one specimen (Figure 8G), which is very similar to Goniodoris sp. 7 in Gosliner et al. Trapania armilla (Figure $8 \mathrm{H}$ ) was collected on a gray sponge in shallow water, whereas T. safracornia (Figure 9A) was collected in deeper areas. 

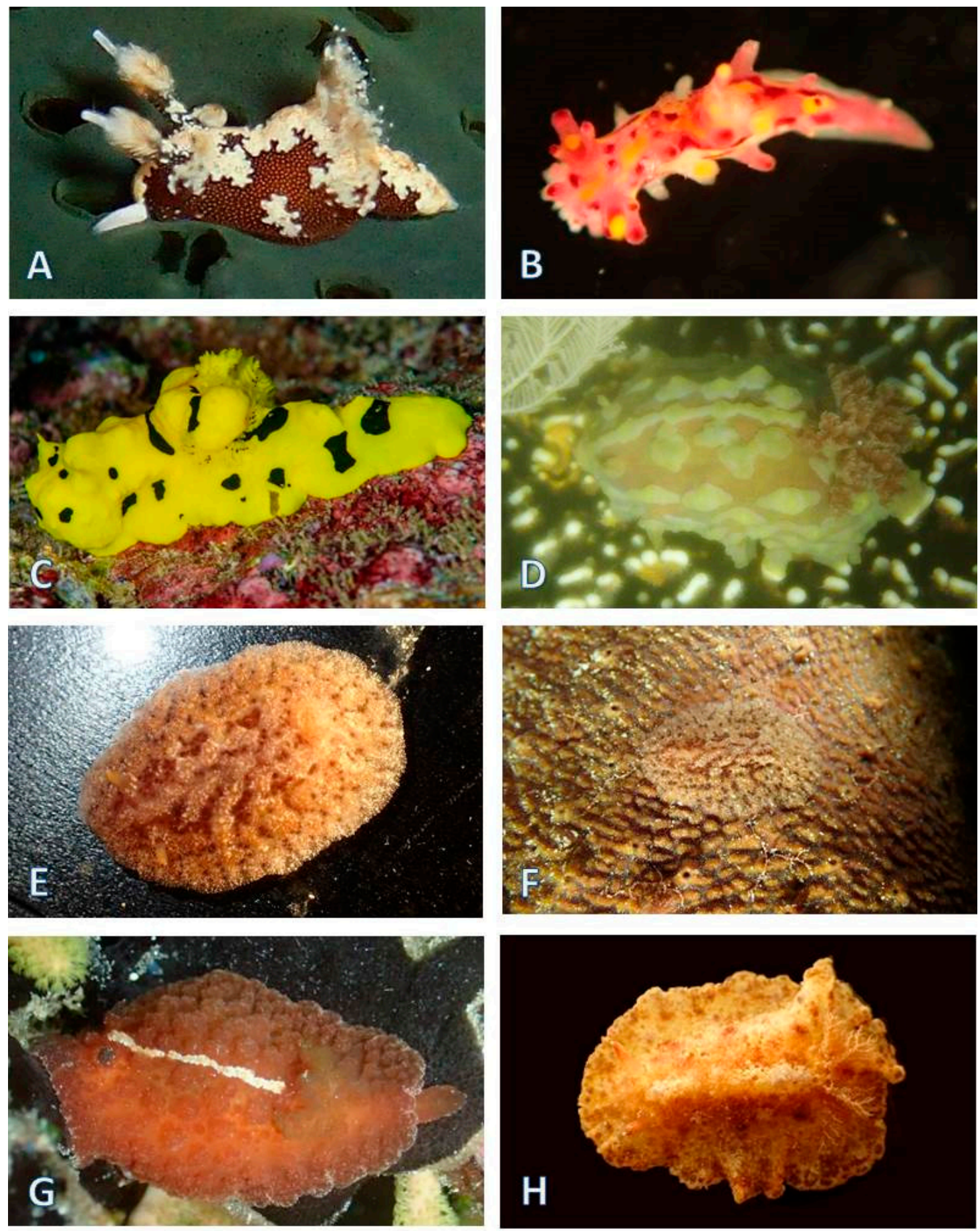

Figure 9. Nudibranchia Doridina: (A) Trapania safracornia*; (B) Aegires sp. (Aegires sp. 7 in Gosliner et al. [24]: 149), Aesp7-18Ba-1; (C) Notodoris minor*; (D) Asteronotus cespitosus*; (E) Asteronotus mimeticus, Asmi18Ba-2; (F) Asteronotus mimeticus substrate, Asmi18Ba3S; (G) Atagema intecta, Atin18Ba-2; (H) Discodoris cebuensis, Dice18Ba-1. *Specimen not collected.

Aegiridae: Both acknowledged aegirid genera were present in our collections: Aegires sp. (Figure 9B), depicted in Gosliner et al. [24] as Aegires sp. 7, and the "banana sea slug" Notodoris minor (Figure 9C) (the latter only by photo-documentation).

Discodorididae: The large and easily identifiable Asteronotus cespitosus (Figure 9D) was only recorded by photo-documentation. Asteronotus mimeticus (Figure 9E) is always associated with a 
phyllospongian sponge. It usually sits underneath and was more often observed during the night. The coloration of the sea slug matches perfectly with the color and structure of the sponge surface (Figure 9F). Two specimens of Atagema intecta (Figure 9G) were collected during night dives, one from sand substrate, the other on a sponge. Two specimens of Discodoris cebuensis (Figure 9H) were also collected during a night dive. Halgerda batangas (Figure 10A) is a common species in this location, while in other areas (e.g., BNP) the species is quite rare. The same is true for Halgerda $c$. (Figure 10B), represented by two specimens. Jorunna funebris (Figure 10C) was common in mangroves, and not all of the about 20 specimens seen were collected. Paradoris liturata (Figure 10D) is a member of the Discodorididae that mimics Phyllidiella pustulosa, illustrated in Figure 10E. Paradoris liturata has tubercles on the notum with black lines in between, as well as black rhinophores, similar to P. pustulosa. When the gills in P. liturata are retracted into the gill pockets, the opening looks like the anus of a Phyllidiella species. Platydoris sanguinea (Figure 10F) was found on coral rubble at $5.5 \mathrm{~m}$ depth.

Chromodorididae: This family is represented in our study by 263 specimens, which could be assigned to 37 species. Ceratosoma tenue (Figure 10G) was only identified by photo-documentation. Eight species of the genus Chromodoris are present in our collection. Chromodoris dianae (Figure 10H), a very common species in BNP, was only represented by two specimens, collected at depths in the range of 13-15 m. Of all chromodorids, C. annae (Figure 11A,B) is the species with the highest specimen numbers and distribution (Table 2); rhinophores and gills vary in color from light yellow to a rather unusual orange. Chromodoris elisabethina (Figure 11C) is recorded only by photographs from Sempini. Chromodoris lochi (Figure 11D,E), the second most common Chromodoris species, is represented by two color morphs: rhinophores and gills are either pink or pale yellow, and the mantle is always translucent white. Molecular barcoding has confirmed both their identities as $C$. lochi. The same two color morphs are also found in BNP [16]. One specimen of $C$. magnifica (Figure 11F) with the typical whitish background was found on sand underneath coral rubble. Another specimen was collected that looked in general very similar to C. magnifica with a discontinuous dark line in the middle of the notum, but with a distinct bluish body. According to molecular barcoding, both genes (CO1 and 16S) show a $99 \%$ similarity with sequences from NCBI assigned to C. quadricolor (Figure 11G). This would then indicate another record in the Indo-Pacific Ocean of a species that is primarily distributed in the Red Sea. Two specimens of $C$. strigata (Figure $11 \mathrm{H}$ ) were collected at depths of 7-14 m, one during a night dive crawling on the green alga Halimeda. Only two specimens of $C$. willani (Figure 12A) were found close to each other at $8 \mathrm{~m}$ depth, another species much more common in BNP. Four specimens of Doriprismatica atromarginata (Figure 12B,C) with sizes of 17-74 mm were collected from different dive sites. In one of these dive sites, we observed a yellow flatworm Pseudoceros sp. (Figure 12D), which clearly mimics D. atromarginata. Only one specimen of $D$. sibogae (Figure 12E) was found, differing from D. atromarginata in gill color. Glossodoris cincta is a common species and widespread in the Indo-Pacific, and is also recorded from BNP, Sangihe, and Lembeh Strait. However, there are several species with very similar coloration [32]. We assign two specimens to this species (Figure 12F). One was collected at $6 \mathrm{~m}$ depth during a night dive underneath coral rubble, and one at $1 \mathrm{~m}$ depth. Both animals had a yellowish to green band followed by a black and then a white margin on the mantle (typical feature of G. cincta). Glossodoris rufomarginata (Figure 12G) was identified only by photo-documentation. It is described in the literature as having an orange-brown spackled pattern on the notum, brown marginal band, and white submarginal band [24]. The genus Goniobranchus was represented by six different species. Goniobranchus coi (Figure 12H) was only collected in 2017 during a night dive. It was not recorded from BNP [16] or Sangihe [18]. Goniobranchus fidelis (Figure 13A) is a smaller member of the genus, and our single animal was only $13 \mathrm{~mm}$ in length, collected at $17 \mathrm{~m}$ depth. Goniobranchus geometricus (Figure 13B) is more common in North Sulawesi, and we collected six specimens at $5-15 \mathrm{~m}$ depth. G. kuniei (Figure 13C) and G. reticulatus (Figure 13D) were collected at deeper than $20 \mathrm{~m}$ depth. Yonow [3] outlines the problems of correct identification of this species, and misidentification of $G$. inopinata and even G. tinctorius cannot be excluded: G. tinctorius usually has a yellow band along the edge of the notum [33], which is not present in our specimens. 

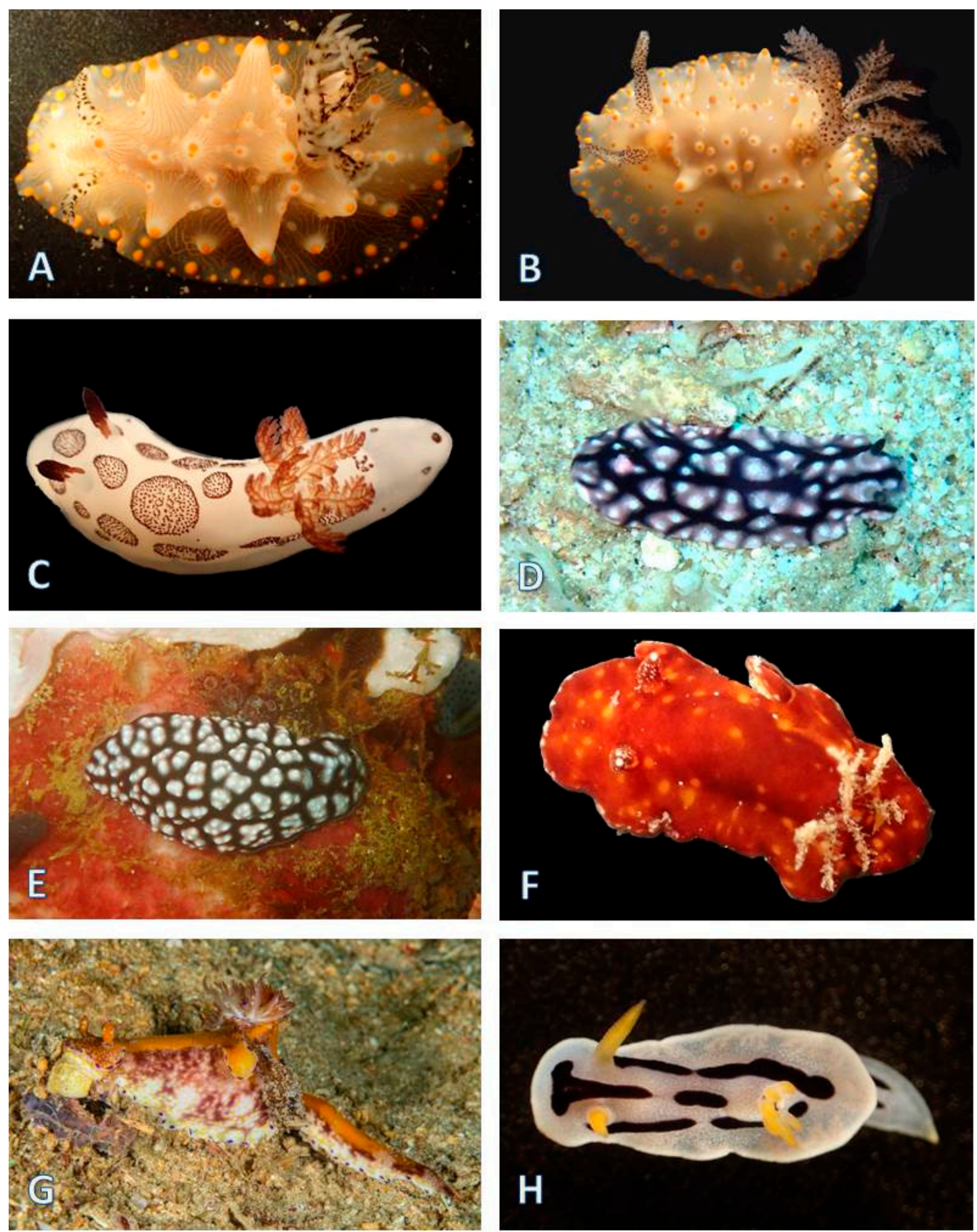

Figure 10. Nudibranchia Doridina: (A) Halgerda batangas, Haba18Ba-8; (B) Halgerda carlsoni, Haca17Ba-2; (C) Jorunna funebris, Jofu17Ba-1; (D) Paradoris liturata in situ, Phpu17Ba-6; (E) Phyllidiella pustulosa mimicked by Paradoris liturata; (F) Platydoris sanguinea, Plsa17Ba-1; (G) Ceratosoma tenue $;(\mathbf{H})$ Chromodoris dianae, Chdi18Ba-1. *Specimen not collected. 

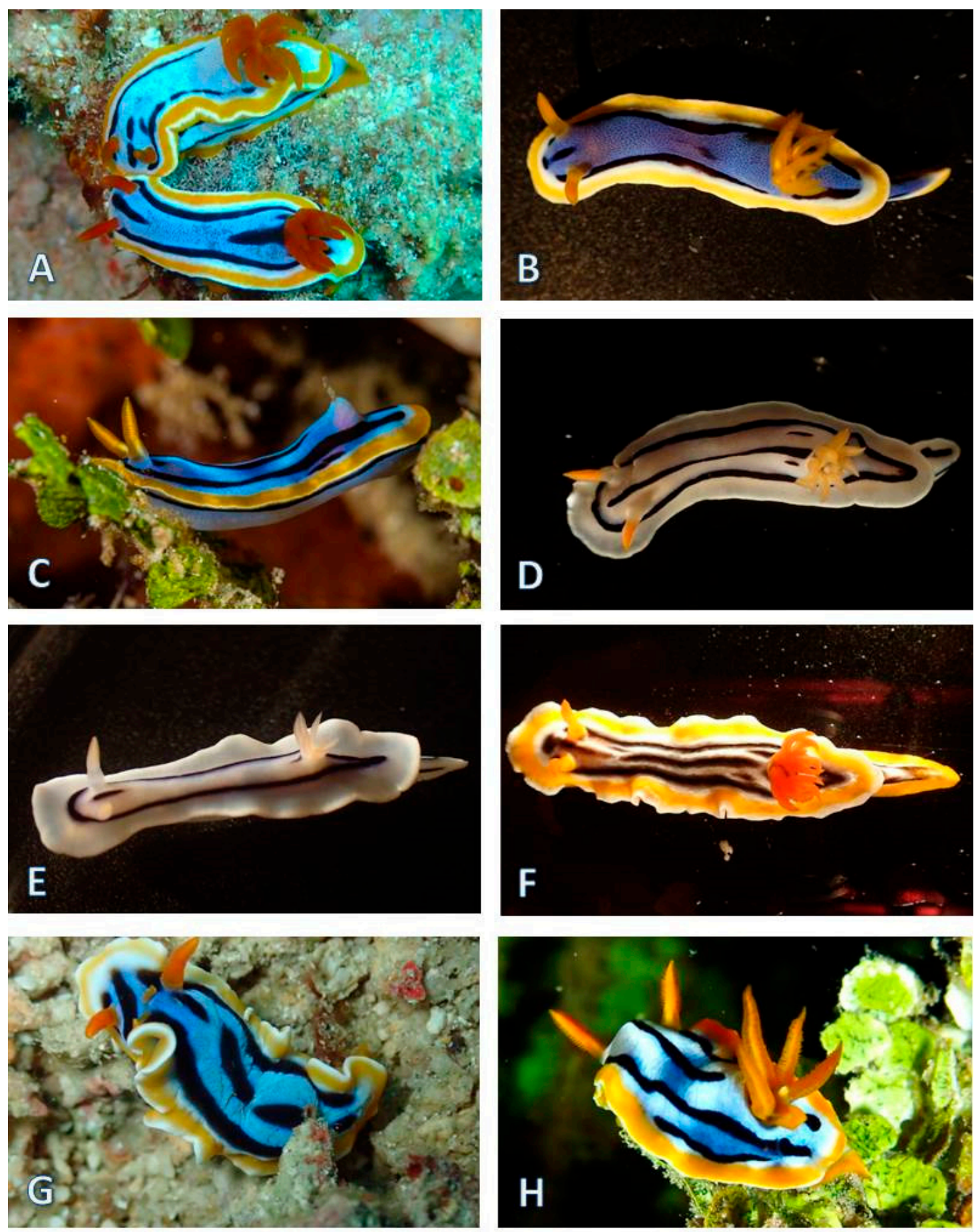

Figure 11. Nudibranchia Doridina: (A,B) Chromodoris annae, Chan18Ba-11-12, Chan18Ba-7; (C) Chromodoris elisabethina*; (D,E) Chromodoris lochi, Chlo18Ba-6, Chlo18Ba-1; (F) Chromodoris magnifica, Chma18Ba-1; (G) Chromodoris quadricolor, Chma18a-2; (H) Chromodoris strigata, Chst17Ba-1. *Specimen not collected. 

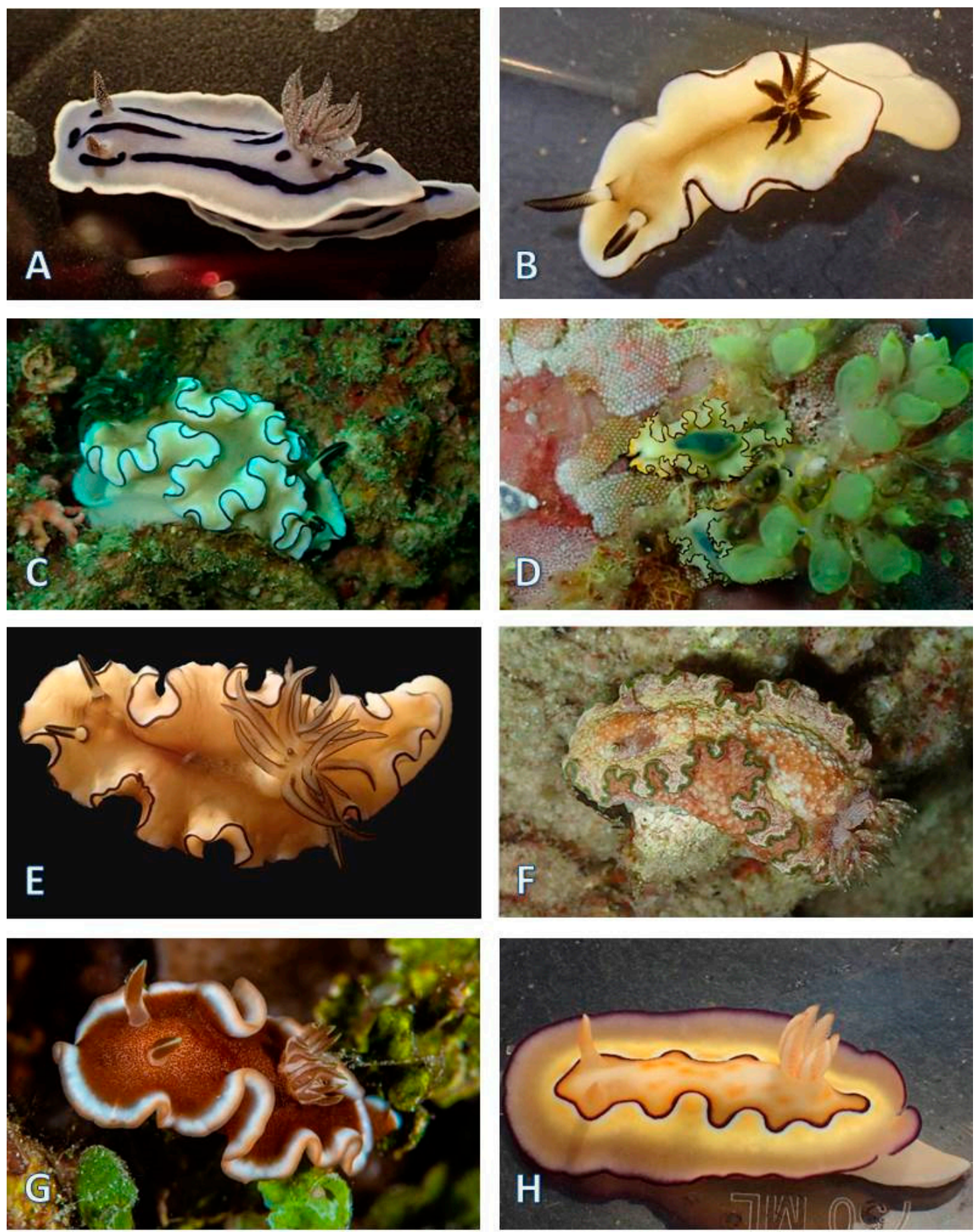

Figure 12. Nudibranchia Doridina: (A) Chromodoris willani, Chwi18Ba-1; (B) Doriprismatica atromarginata, Doat17Ba-1; (C) Doriprismatica atromarginata in situ; (D) Flatworm mimicking D. atromarginata; (E) Doriprismatica sibogae, Dosi17Ba-1; (F) Glossodoris cincta, Glci18Ba-1; (G) Glossodoris rufomarginata*; (H) Goniobranchus coi, Gocho17Ba-1. *Specimen not collected. 

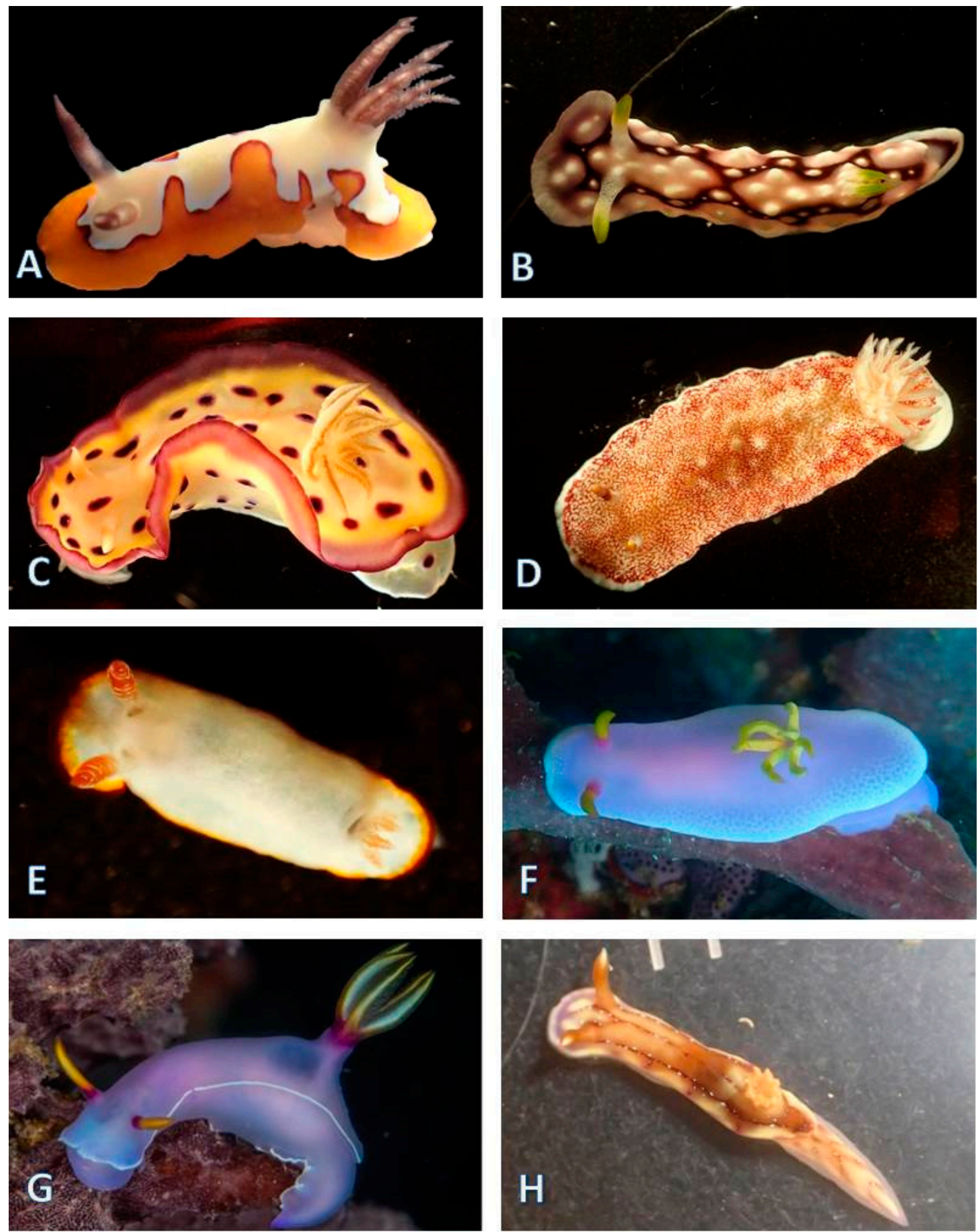

Figure 13. Nudibranchia Doridina: (A) Goniobranchus fidelis, Gofi17Ba-1; (B) Goniobranchus geometricus, Goge18Ba-5; (C) Goniobranchus kuniei, Goku18Ba-1; (D) Goniobranchus reticulatus, Gore18Ba-1; (E) Goniobranchus verrieri, Thho18Ba-1; (F) Hypselodoris apolegma, Hyap17Ba-1; (G) Hypselodoris bullockii*; (H) Hypselodoris cerisae, Hysp1.17Ba-1. *Specimen not collected.

In contrast to BNP, where only five species have been recently depicted [16], 12 species of Hypselodoris are recorded in Bangka Archipelago: H. apolegma (Figure 13F), H. bullockii (Figure 13G), H. cerisae (Figure 13H), H. decorata (Figure 14A), H. emma (Figure 14B), H. iacula (Figure 14C), H. lacuna (Figure 14D), H. maculosa (Figure 14E), H. maridadilus (Figure 14F), H. zephyra (Figure 14G), H. tryoni (Figure 14H) and Hypselodoris sp. a (Figure 15A). Many of the species are easily recognized by their 
distinct coloration and are not discussed in detail below. A recently described new species, $H$. $i b a$, is very similar to H. bullockii; however, our animal, which was only photo-documented (Figure 13G), shows the features typical of H. bullockii also found in Ambon [5] and BNP [16]. However, confirmation of the identification of this individual would require specimens and possibly molecular analyses. One member of the newly described species H. cerisae (Figure 13H) [27] was collected in front of Coral Eye, and molecular analyses verified the identification based on $99 \%$ similarity of our specimen with the sequence of $H$. cerisae CASIZ 178350 deposited in NCBI. Our specimen is pale brown with some purple pigment concentrated at the anterior mantle and foot margin; white dots are aligned on three longitudinal brown lines on the notum; brownish diagonal lines are present on the posterior foot; there are nine gill branches. Externally, H. decorata (Figure 14A) is very similar to H. maculosa (Figure 14E). According to recent studies [27], they are closely related and form a clade with $H$. juniperae. H. decorata can be distinguished from $\mathrm{H}$. maculosa by the three reddish rhinophoral bands, versus only two reddish rings. Our specimen of $H$. decorata has these three rhinophoral rings, as well as the other typical color features, e.g., the white dots on the broad brown margin of the mantle and longitudinal lines on the middle part of the mantle with brown dots. Our single specimen of $H$. iacula (Figure 14C) has a light honey-colored body, with a brown band around the mantle margin, violet at the foot margin, and a white net-like pattern on the notum and top of the foot. Eight gill branches arise from a relatively high gill pocket. Two specimens are assigned to H. lacuna (Figure 14D), whose name is based on the translucent areas on the notum that resemble holes in the body wall [27]. Both specimens have a white mantle, with many translucent patches and two large translucent circles posterior to the rhinophores with two others in front of the gills; further characteristics are blue dots on the lateral margin of the mantle, and white gills and rhinophores with reddish apices. The body of Hypselodoris sp. a (Figure 15A) is cream, as well as the rhinophores, which are more translucent at the base and with one orange ring in the cream area. Red dots spread along the margin of the notum and a few along the midline of the notum. Externally, the species is very similar to Thorunna australis and Hypselodoris maculosa. However, Hypselodoris sp. a lacks contrasting pigment in the middle of the notum, whereas H. maculosa shows dots all over the notum. Two specimens of Mexichromis aurora (Figure 15B) were sitting next to each other on corals at $8.6 \mathrm{~m}$ depth, but only one was collected. $M$. trilineata (Figure 15C) was only identified by photo-documentation.

One small animal (Dorid18Ba-1; Figure 15D) was found in Yellow Coco at a depth of $20 \mathrm{~m}$. It is characterized by a pale blue body color with an orange mantle rim, orange gills and rhinophores, as well as orange patches on the dorsal notum and tail. The body shape resembles some goniodorids. However, it could be identified as a juvenile of the species Miamira magnifica. One further small member of probably the same genus, Miamira sp. a (Figure 15E) $(10 \mathrm{~mm})$, was found at a depth of $4 \mathrm{~m}$. It is reddish to brown in color, covered with darker spots and many tiny white dots on the mantle; the edge of the mantle is cream with brown dots. The rhinophores are translucent cream with white lines and an orange hue at the tip, whereas the translucent gills exhibit brown and white patches. Verconia simplex (Figure 15F), $6 \mathrm{~mm}$ in size, was sitting on black sponges at $5.2 \mathrm{~m}$ depth. Both specimens of Thorunna furtiva (Figure 15G) were found close to each other on a stone, covered by encrusting red sponge, and were observed to have vibrating gills, a typical character of the genus. Dendrodoris nigra (Figure $15 \mathrm{H}$ ) is the only member of the family Dendrodorididae that was collected. It is a juvenile of only $6 \mathrm{~mm}$ length and was found crawling out of the coral rubble sorted in the laboratory. 

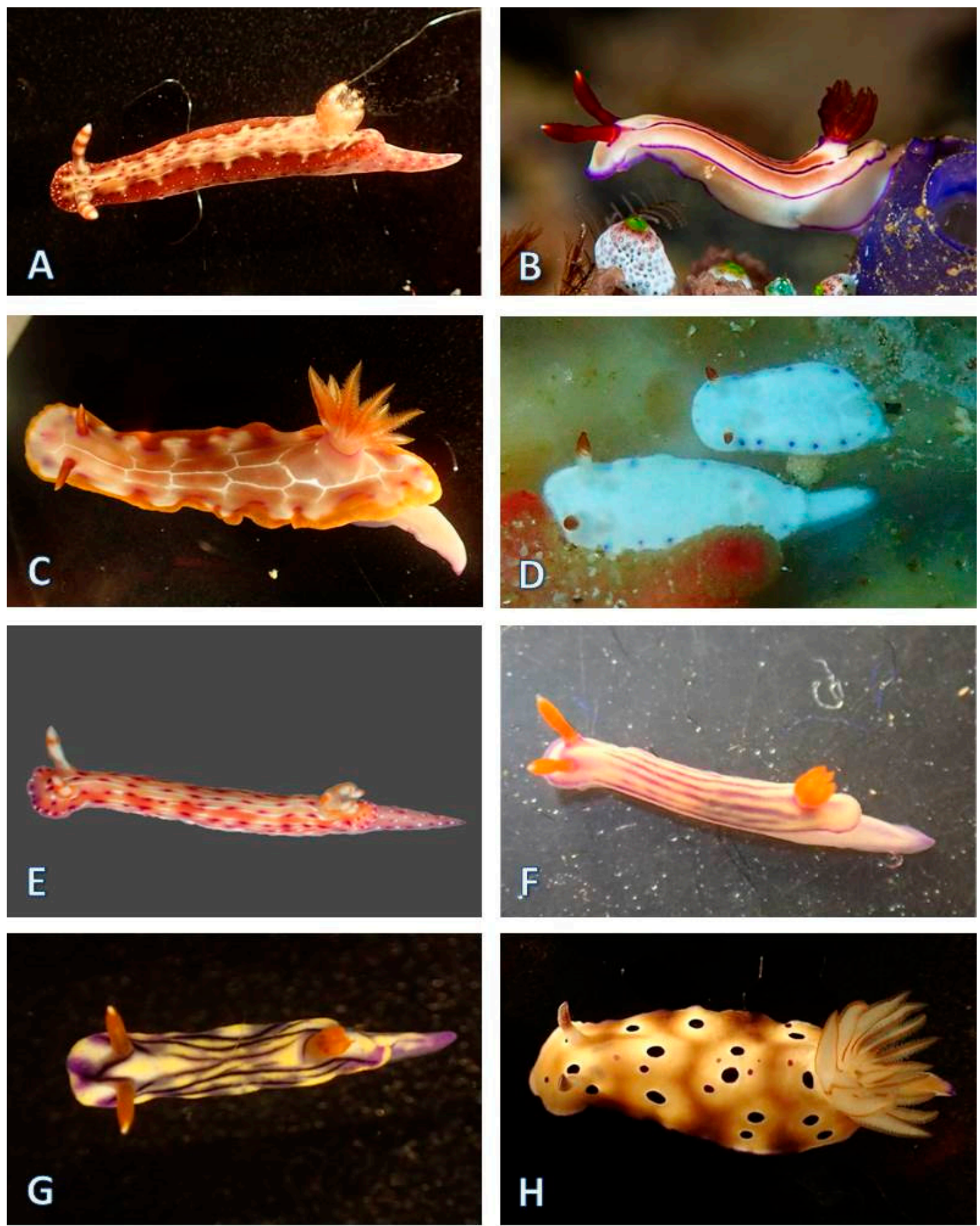

Figure 14. Nudibranchia Doridina: (A) Hypselodoris decorata, Hyma18Ba-1; (B) Hypselodoris emma*; (C) Hypselodoris iacula, Chromos18Ba-1; (D) Hypselodoris lacuna, Hysp19_17Ba-1-2; (E) Hypselodoris maculosa, Hyma17Ba-1; (F) Hypselodoris maridadilus, Hymari17Ba-1; (G) Hypselodoris zephyra, Hyni18Ba-1; (H) Hypselodoris tryoni, Hytr18Ba-5. *Specimen not collected. 

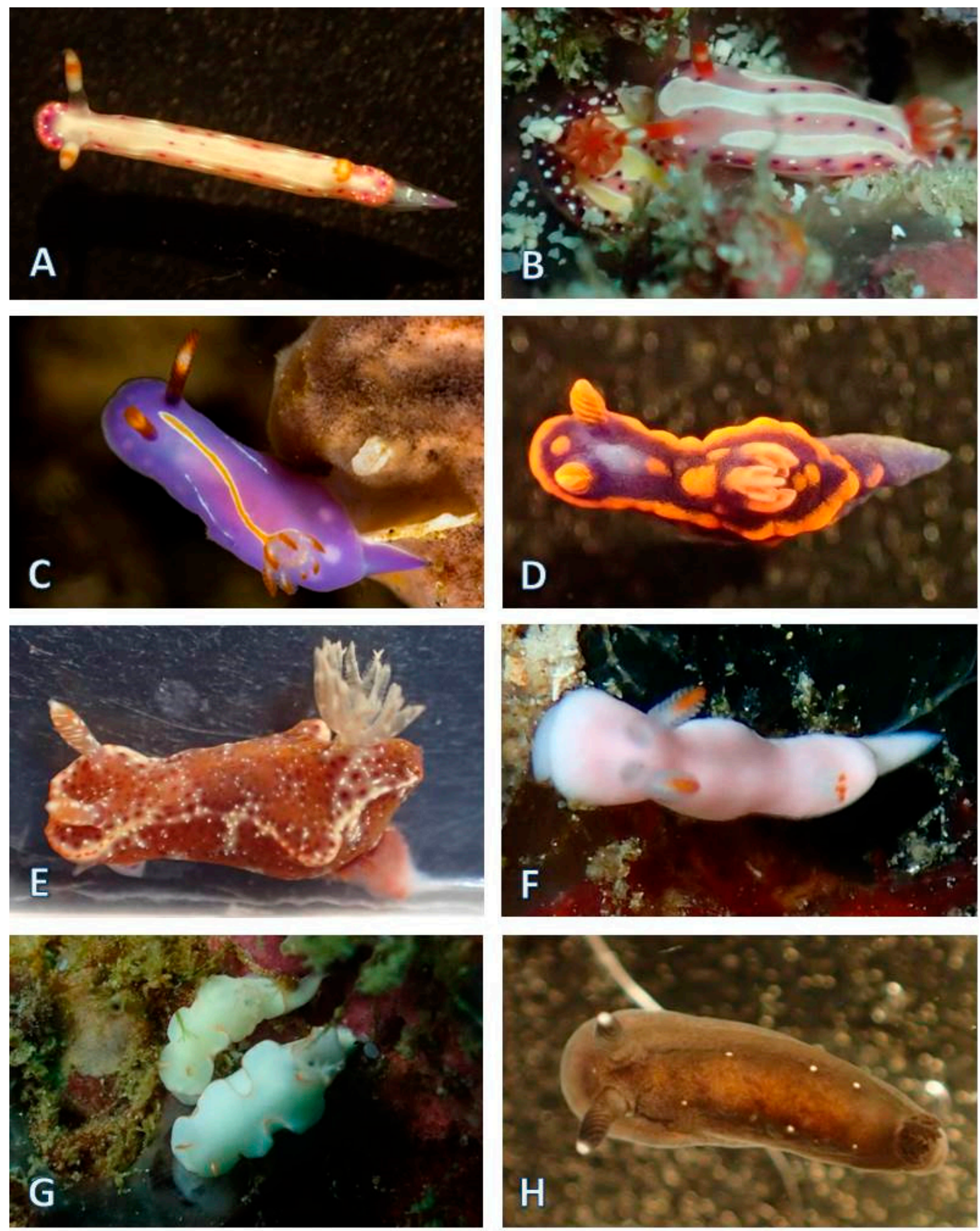

Figure 15. Nudibranchia Doridina: (A) Hypselodoris sp. a, Thsp1-18Ba-1; (B) Mexichromis aurora, Meau18Ba-1; (C) Mexichromis trilineata*; (D) Miamira magnifica, Dorid18Ba-1; (E) Miamira sp. a, Misp17Ba-1; (F) Verconia simplex, Nosi17Ba-1; (G) Thorunna furtiva, Thfu18Ba-1-2; (H) Dendrodoris nigra, Deni18Ba-1. *Specimen not collected.

Phyllidiidae: Members of this family are probably the most common sea slugs in tropical waters. More than 100 specimens were collected in the Bangka Archipelago, assigned to 16 species and three genera, Phyllidia, Phyllidiella, and Phyllidiopsis. Phyllidia cf. babai (Figure 16A) was found in Batu Belah and was only identified by photo-documentation. Eight specimens of Phyllidia coelestis (Figure 16B) were collected at 1-20 m depth. The other Phyllidia species represented in our collection are P. elegans (Figure 16C), P. exquisita (Figure 16D), P. ocellata (Figure 16E), and P. picta (Figure 16F). Phyllidia picta 
often co-occurred at the same locations as P. ocellata. Phyllidia varicosa (Figure 16G,H) was probably the most common Phyllidia species with 16 specimens collected at nine sites, ranging between 23 and $85 \mathrm{~mm}$ in size and between 2 and $20 \mathrm{~m}$ in depth. One specimen originally assigned to Phyllidia picta (Phsp18Ba-2; Figure 17A), based on external coloration, clusters with several other specimens from various regions of North Sulawesi in a separate clade, distinct from the P. picta clade. It therefore represents a new species with very similar appearance to P. picta (Papu et al., in preparation). Four species of Phyllidiella collected in Bangka Archipelago comprise Phyllidiella cf. annulata (Figure 17B), P. lizae (Figure 17C), P. nigra (Figure 17D) and P. pustulosa (Figure 17E,F). Only one specimen of Phyllidiella cf. annulata (Figure 17B) was found in Talisei Island, a collection site with strong tidal currents and ocean waves, as well as small compact coral formations. Phyllidiella nigra (Figure 17D) was collected from mangrove roots. It is characterized by the dark gray hyponotum and foot, and black notum with single pale pink round tubercles. Phyllidiella pustulosa (Figure 17E,F) is the most common species of the genus Phyllidiella and also within Phyllidiidae. However, this species was shown to represent a complex of several species [25], which are very difficult to distinguish by external characteristics alone. Figure 17G,H depict a specimen which we removed from the substrate to show feeding marks on the sponge. Phyllidiopsis annae (Figure 18A,B) is a small species, and our four specimens ranged in length from 3 to $12 \mathrm{~mm}$. Further collected Phyllidiopsis species are P. cf. burni (Figure 18C), P. krempfi (Figure 18D,E), and P. xishaensis (Figure 18F).

b. Nudibranchia, Cladobranchia (42 species in 22 genera belonging to 12 families)

Arminidae: Six different species of Dermatobranchus were collected during the two studies: D. caeruleomaculatus (Figure 19A), D. rudmani (Figure 19B), D. pustulosus (Figure 19C), and three undescribed Dermatobranchus species (Figure 19D-F). Most of them were collected in 2018 close to Coral Eye under coral rubble.

Proctonotidae: One Janolus species (Figure 19G) exists only as a photo-documentation in our collection. It is already depicted in Gosliner et al. as Janolus sp. 1.

Bornellidae: Several individuals of Bornella anguilla (Figure 19H) (of which only two were collected) were found crawling on encrusting corals and tunicates, and some were mating at approximately $8 \mathrm{~m}$ depth. One Bornella specimen was found in coral rubble at $1 \mathrm{~m}$ depth, which resembles most $B$. dotoides when comparing the shape of the cerata (Figure 20A) [3].

Tethydidae: Melibe bucephala (Figure 20B) was found together with B. stellifera in the collected coral rubble. Melibe engeli (Figure 20C; length of $34 \mathrm{~mm}$ ) is a well-camouflaged species due to its transparent body. The species is recorded to be associated with algae of the genus Padina or Achantophora [24]; however, our animal was collected from an encrusting sponge.

Dotidae: The brownish-cream to olive-colored species Doto ussi (Figure 20D) was always found at the base of the hydrozoan Aglaophenia cupressina.

Tritoniidae: Two undescribed species were collected, both at the same site, with many different soft coral species close by. The dorsal appendages of the undescribed Marionia species (Figure 20E,F) look like green algae, and the animal is very similar to M. sp. 2, depicted in Gosliner et al. [24]. The dorsal appendages of the undescribed Tritonia sp. (Figure 20G) resemble polyps of soft corals. The animal is similar to T. sp. 3 depicted by Gosliner et al. [24].

Flabellinidae: Coryphellina exoptata (Figure 20H), C. rubrolineata (Figure 21A), and two undescribed species (Flabellina sp. 2, Flabellina sp. 3) are members of the family Flabellinidae. The color of Flabellina sp. 2 (Figure 21B) (also depicted in Gosliner et al. [24]) is similar to C. rubrolineata, but our animal has only one ring around each ceras, and no lines in the middle and margin of the body. The specimen of Flabellina sp. 3 (Figure 21C, similar to F. sp. 3 in Gosliner et al. [24]) has a rather translucent body with a single red line in the middle of the body. The white cerata carry a few red dots, and the rhinophores are translucent with a tinge of white in the middle part. 

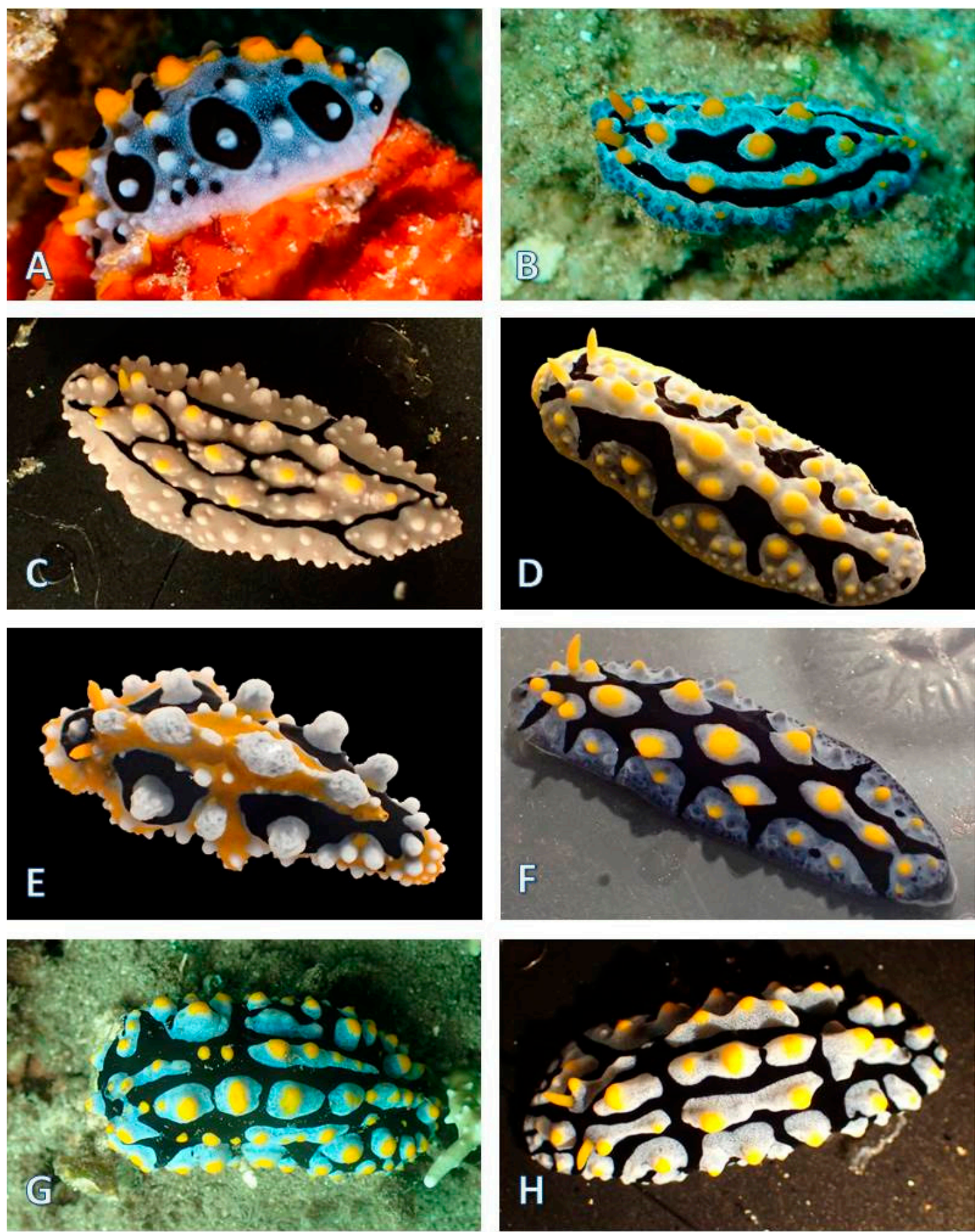

Figure 16. Nudibranchia Doridina: (A) Phyllidia cf. babai*; (B) Phyllidia coelestis, Phco18Ba-1; (C) Phyllidia elegans, Phel18Ba-1; (D) Phyllidia exquisita, Phex17Ba-1; (E) Phyllidia ocellata, Phoc17Ba-1; (F) Phyllidia picta, Phpi17Ba-1; (G,H) Phyllidia varicosa, Phva18Ba-11, Phva18Ba-6. *Specimen not collected. 

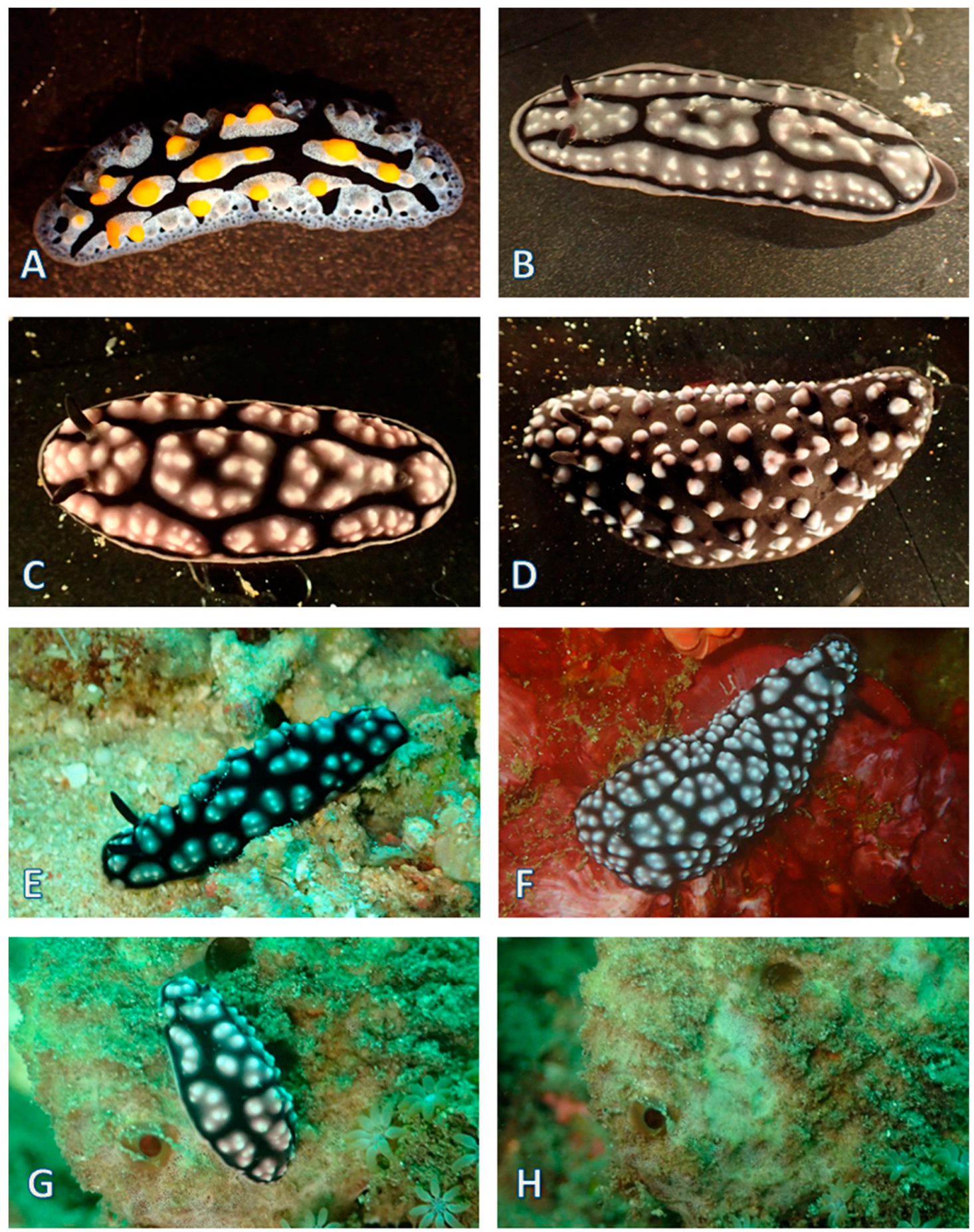

Figure 17. Nudibranchia Doridina: (A) Phyllidia sp. a, Phsp18Ba2; (B) Phyllidiella annulata, Phpu18Ba-12; (C) Phyllidiella lizae, Phli18Ba-1; (D) Phyllidiella nigra, Phni18Ba-1; (E,F) Phyllidiella pustulosa, Phpu18Ba-2, Phpu18Ba-4. (G) Specimen of Phyllidiella pustulosa in situ on a sponge; (H) Sponge viewed immediately after P. pustulosa was removed. 

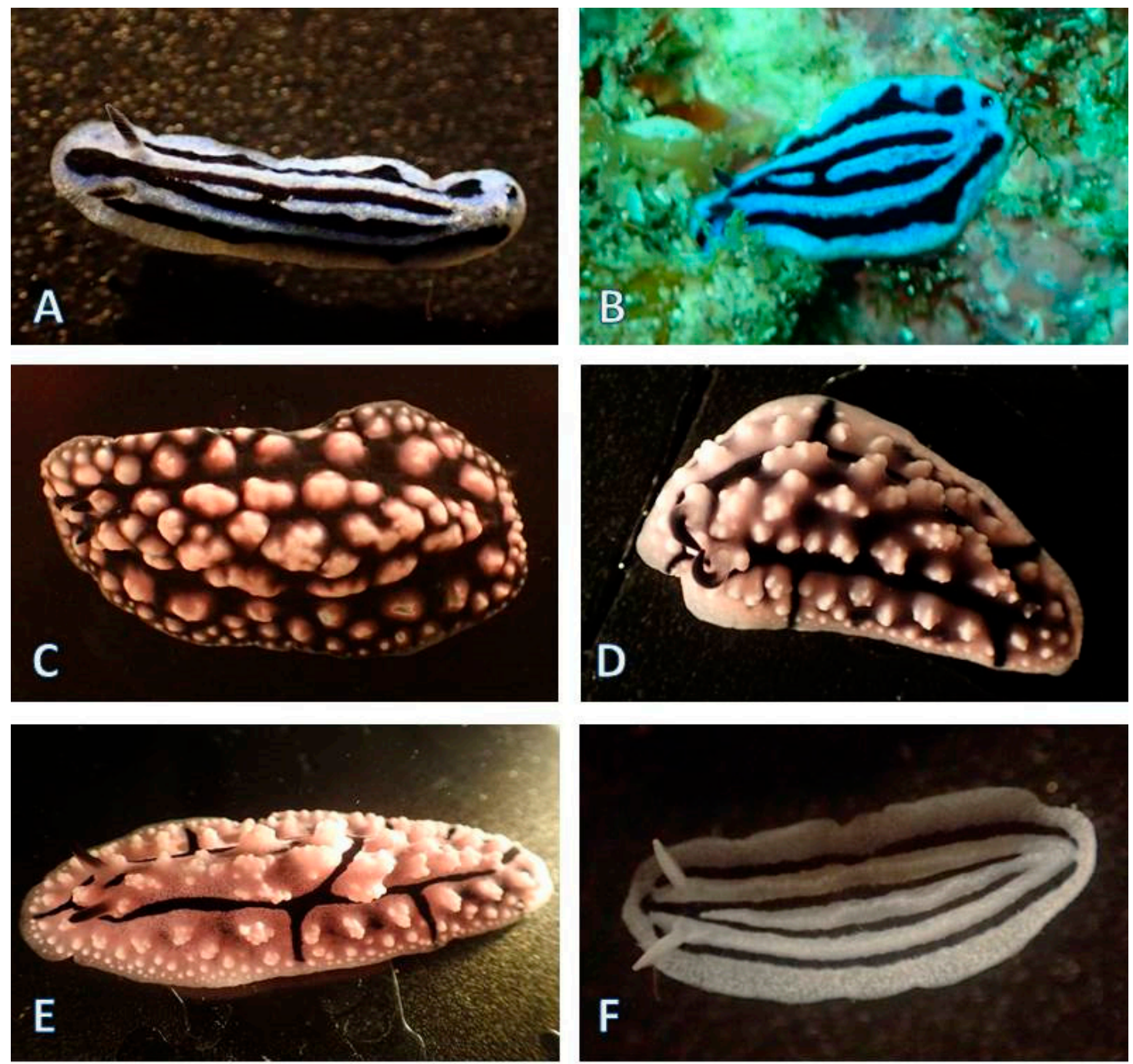

Figure 18. Nudibranchia Doridina: (A,B) Phyllidiopsis annae, Phsan18Ba-1 and in situ; (C,D) Phyllidiopsis cf. burni, Phssp18Ba-1; (E) Phyllidiopsis krempfi, Phskre18Ba-1, Phspi18Ba-1; (F) Phyllidiopsis xishaensis, Phsxi18Ba-1.

Samlidae: Samla riwo (Figure 21D) was collected from a non-specific substrate.

Eubranchidae: Eubranchus sp. 22 (Figure 21E) was only found once, with one specimen on a hydrozoan of the family Plumulariidae [24].

Trinchesiidae: The conspicuous Trinchesia yamasui (Hamatani, 1993) (Figure 21F) was only recorded by photo-documentation. It is very similar to the original and subsequent descriptions $[21,34,35]$. The animal is grayish, with a black head followed by a violet band in front of the rhinophores. The cerata are black to gray, with a subapical orange ring, followed by a black apex. Two specimens of an undescribed Cuthona species (Figure 21G) depict a uniformly orange body color with the oral tentacles and rhinophores showing a darker orange on the distal parts, followed by a translucent apical part. The specimens resemble Cuthona sp. 57 in Gosliner et al. [24]. One unidentified aeolid specimen (Aeol18Ba-1; Figure 21H) was collected from coral rubble and is characterized by a pale white body and cerata with the digestive gland shining through in a darker shade. 

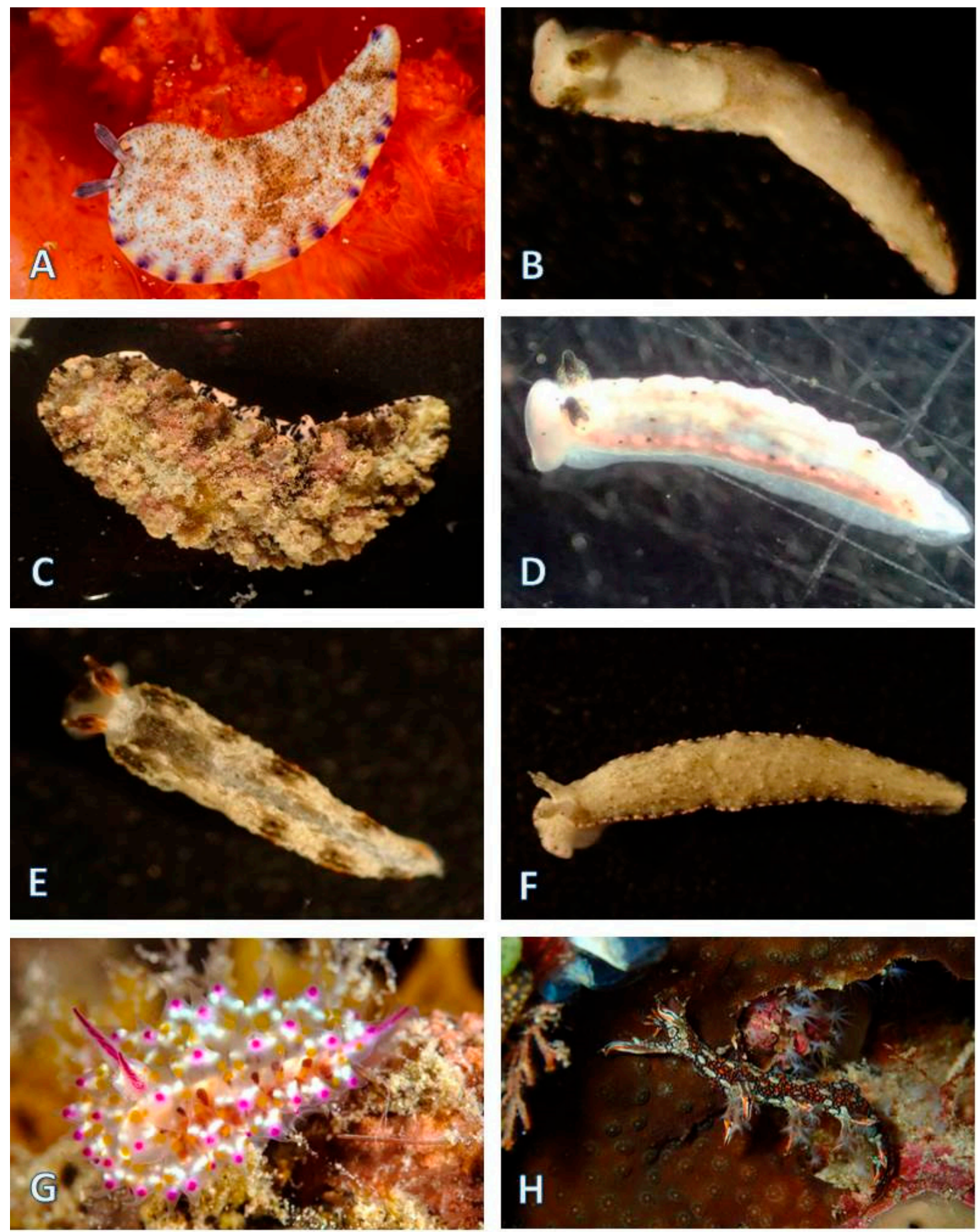

Figure 19. Nudibranchia Cladobranchia: (A) Dermatobranchus caeruleomaculatus*; (B) Dermatobranchus rodmani, Dero18Ba-1; (C) Dermatobranchus pustulosus, Depu18Ba-1; (D) Dermatobranchus sp. a, Desp.a18Ba-1; (E) Dermatobranchus sp. b, Desp18Ba-1; (F) Dermatobranchus sp. c, Desp17Ba-1; (G) Janolus sp. (Janolus sp. 1 in Gosliner et al. [24]: 305)*; (H) Bornella anguilla, Boan17Ba-1. *Specimen not collected. 

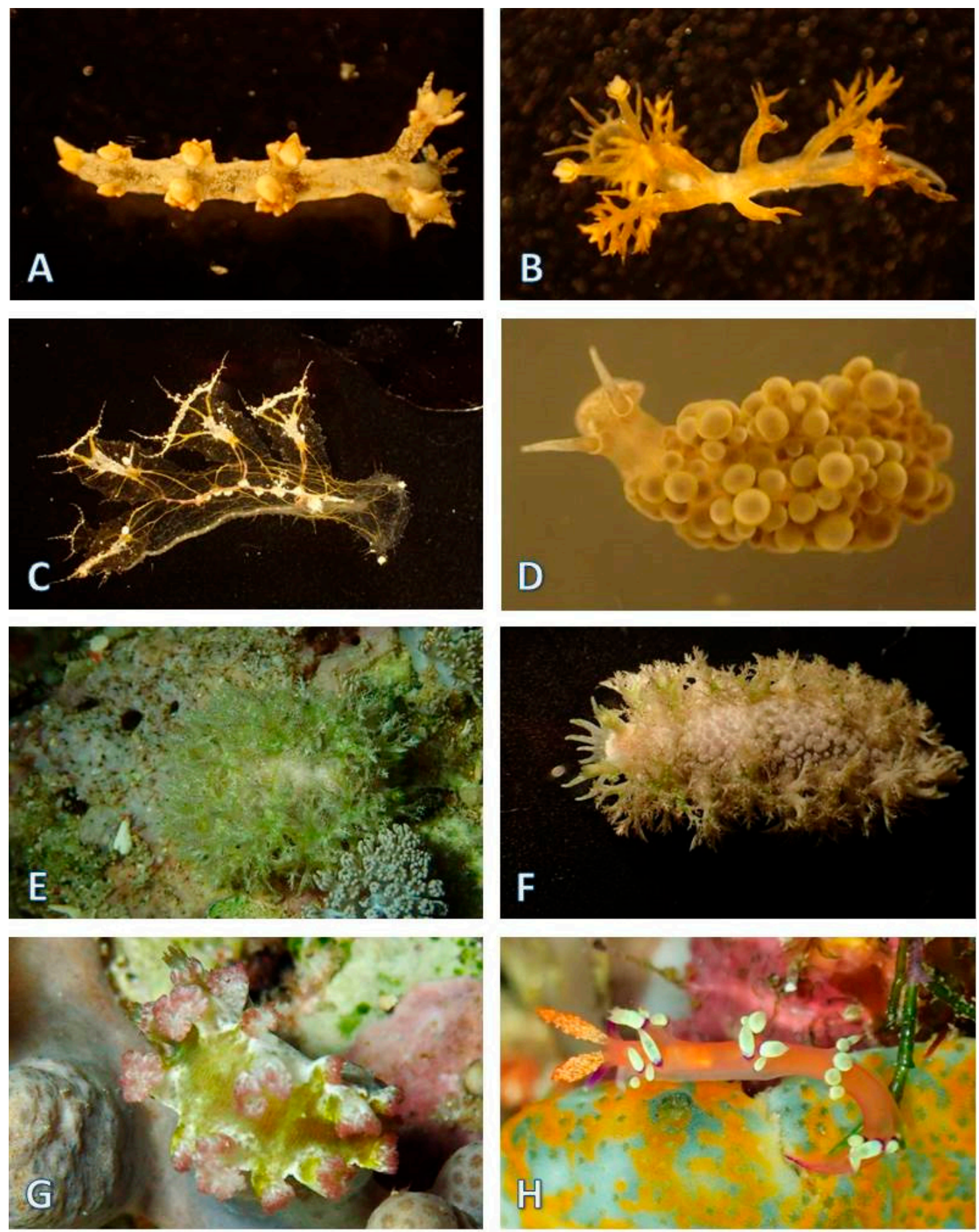

Figure 20. Nudibranchia Cladobranchia: (A) Bornella dotoides, Bohe18Ba-1; (B) Melibe bucephala, Mesp.a18Ba-1; (C) Melibe engeli, Mebu18Ba-1; (D) Doto ussi, Dous18Ba-2; (E,F) Marionia sp. (Marionia sp. 2 in Gosliner et al. [24]: 324), Masp2.18Ba-1; (G) Tritonia sp. (Tritonia sp. 3 in Gosliner et al. [24]: 320), Trsp3_17Ba-1; (H) Coryphellina exoptata, Flex17Ba-1. 

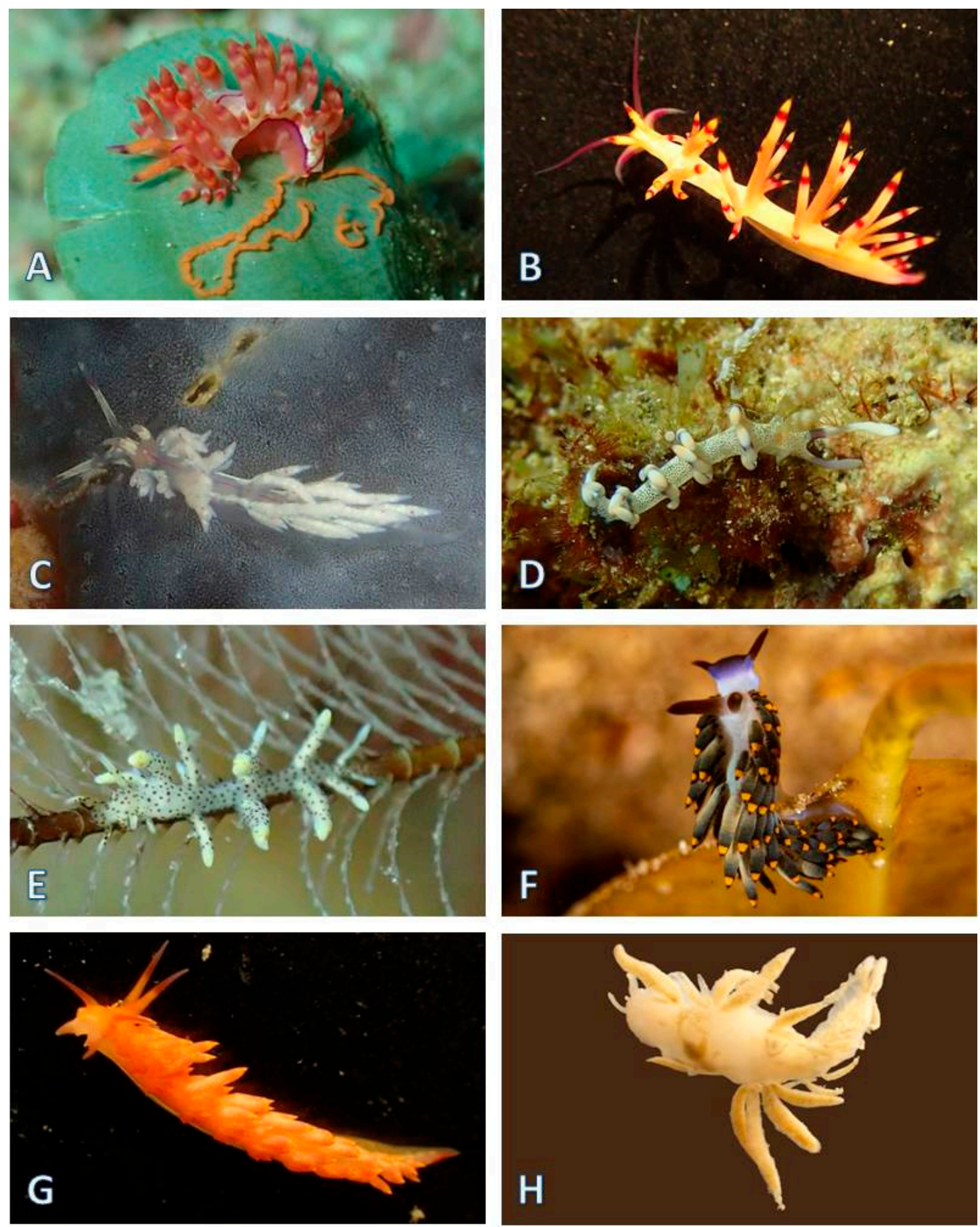

Figure 21. Nudibranchia Cladobranchia: (A) Coryphellina rubrolineata, Flru17Ba-1; (B) Flabellina sp. (Flabellina sp. 2 in Gosliner et al. [24]: 333), Flsp2-18Ba-1; (C) Flabellina sp. (Flabellina sp. 3 in Gosliner et al. [24]: 333), Flsp3-17Ba-1; (D) Samla riwo, Flri17Ba-3; (E) Eubranchus sp. (Eubranchus sp. 22 in Gosliner et al. [24]: 341), Eusp22-18Ba-1; (F) Trinchesia yamasui*; (G) Cuthona sp. (Cuthona sp. 57 in Gosliner et al. [24]: 353), Cusp57-18Ba-1; (H) Aeolidia sp. A, Aeol18Ba-1. *Specimen not collected. 
Facelinidae: This cladobranch family is represented by the highest number of specimens (99) and species (20) in this study. Several new species were also recorded. One specimen probably represents an undescribed Antonietta species (Figure 22A). It measured only $2 \mathrm{~mm}$ alive and has an opaque white body, violet cerata with a dark violet ring on each ceras, and yellow rhinophores. One of the most common facelinid species is Caloria indica (Figure 22B), which we collected at depths of 5-14 m. An unidentified specimen probably belonging to the genus Cratena (Figure 22C) was collected during a night dive at $4 \mathrm{~m}$ depth. It is characterized by the orange color of the jaws, a typical character of the genus. Many members of Favorinus collected during this study were observed feeding on other nudibranch egg masses. F. japonicus (Figure 22D) was collected from the macroalga Udotea while laying eggs. Favorinus sp. (Figure 22E) was also found on Udotea, feeding on a large nudibranch egg mass. Our animal is similar to F. sp. 1 in Gosliner et al. [24]. F. tsuruganus (Figure 22F) was collected from coral rubble. Two specimens of an undescribed Moridilla species (Figure 22G,H) were collected in 2017 and 2018. Both of these specimens are white, with white and yellow on the oral tentacles. The cerata are shorter than the rhinophores, which carry papillae along the posterior side. Molecular analyses clearly distinguish these specimens as a separate species and group them as sister taxa to a new Moridilla species recently described from Bunaken National Park, M. jobeli [36]. The animals exhibit an interesting behavior of thrusting and wriggling the cerata when disturbed, and swimming with lateral movements. Three undescribed Noumeaella species were collected: one looks very similar to Noumeaella sp. 2 depicted in Gosliner et al. [24] (Figure 23A), the second resembles Noumeaella sp. 3 (Figure 23B) [24], and the third is similar to Noumeaella sp. 13 (Figure 23C) [24]. The genus Phyllodesmium was represented in our collection by eight species. P. briareum (Figure 23D) is usually found on the soft coral Briareum, where the cerata are very well camouflaged by the tentacles of the octocoral. P. cf. crypticum (Figure 23E) was found while laying an egg mass at the base of an unidentified xeniid soft coral species. Three specimens of $P$. lizardense (Figure 23F) were collected at various depths, down to $10 \mathrm{~m}$. One large P. longicirrum (Figure 23G; $125 \mathrm{~mm}$ ) was collected on the sand flat at Efrata site at $25 \mathrm{~m}$ depth. It usually feeds on the soft coral Sarcophyton, which was also quite common on that sandy flat. P. magnum (Figure 22H) and P. parangatum (Figure 24A) were collected from members of the family Xeniidae, while P. pecten (Figure 24B) was extracted from coral rubble. Two specimens of $P$. poindimiei (Figure 24C,D) were collected from an unspecific substrate. Pteraeolidia semperi (Figure 24E) is one of the largest facelinid species and was the most common cladobranch species observed, due to an aggregation of more than 100 specimens in shallow water (Sempini), probably for mating.

\subsubsection{Eupulmonata (One Species in One Genus Belonging to One Family)}

Onchidiidae: One member of this family, an undescribed Peronia species (confirmed by barcoding) (Figure 24F), was collected in the mangrove. It lives in tidal areas and was nearly $40 \mathrm{~mm}$ in size. The notum is a cream background color, with pale green and orange patches. Small cream-colored tubercles are spread over the notum.

Figure 25 provides an overview of the species numbers in the various sampling sites, taking into consideration the number of sampling events per site. The high sampling effort at Coral Eye contributed considerably to overall species numbers in this area. 

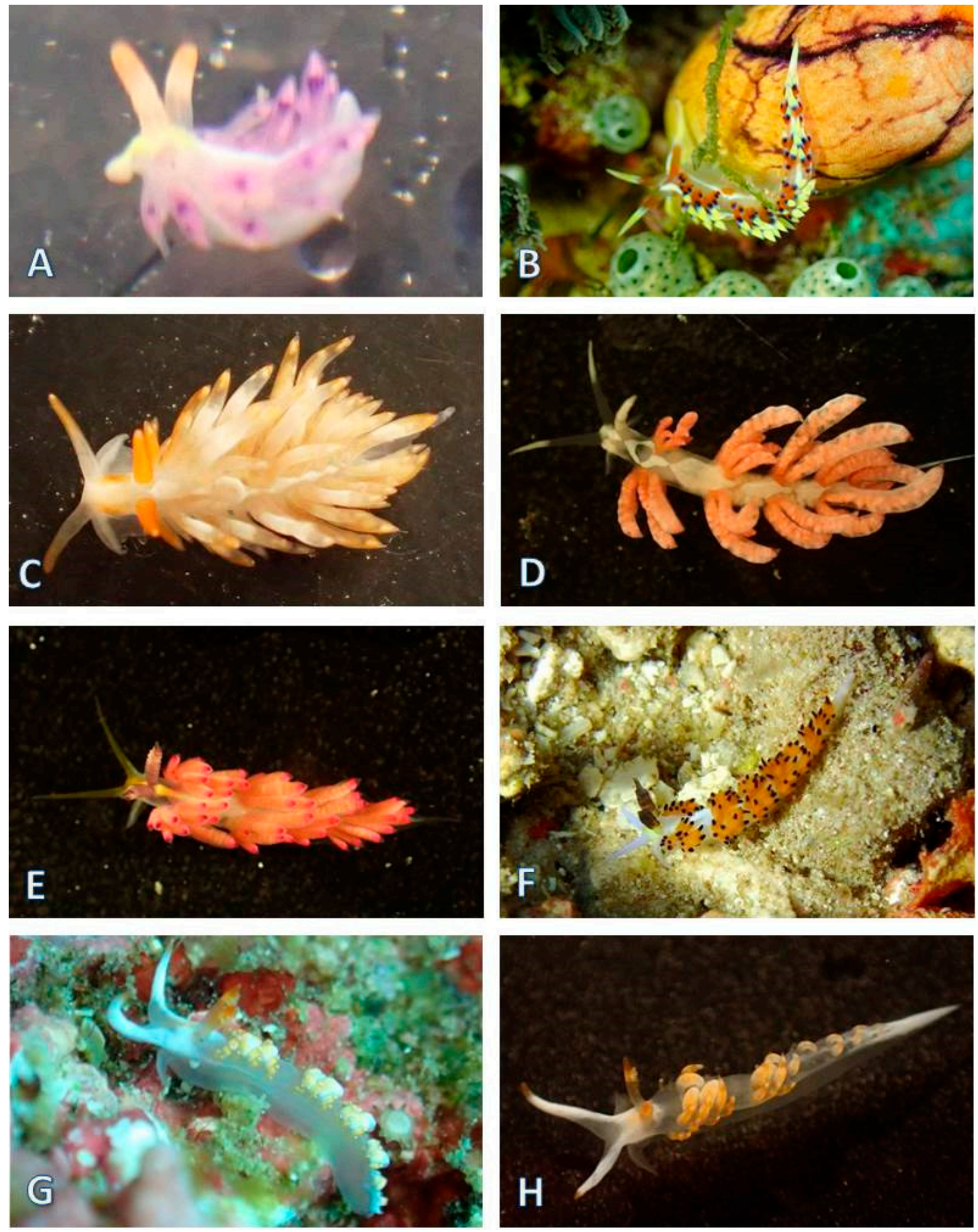

Figure 22. Nudibranchia Cladobranchia: (A) Antonietta sp. a, Ansp17Ba-1; (B) Caloria indica, Cain18Ba-3; (C) Cratena sp. a, Crsp17Ba-1; (D) Favorinus japonicas, Faja18Ba-1; (E) Favorinus sp. (Favorinus sp. 1 in Gosliner et al. [24]: 363), Fasp1.18Ba-1; (F) Favorinus tsuruganus, Fats17Ba-1; (G,H) Moridilla sp. a, Nosp1.17Ba-1, Mojo18Ba-1. 

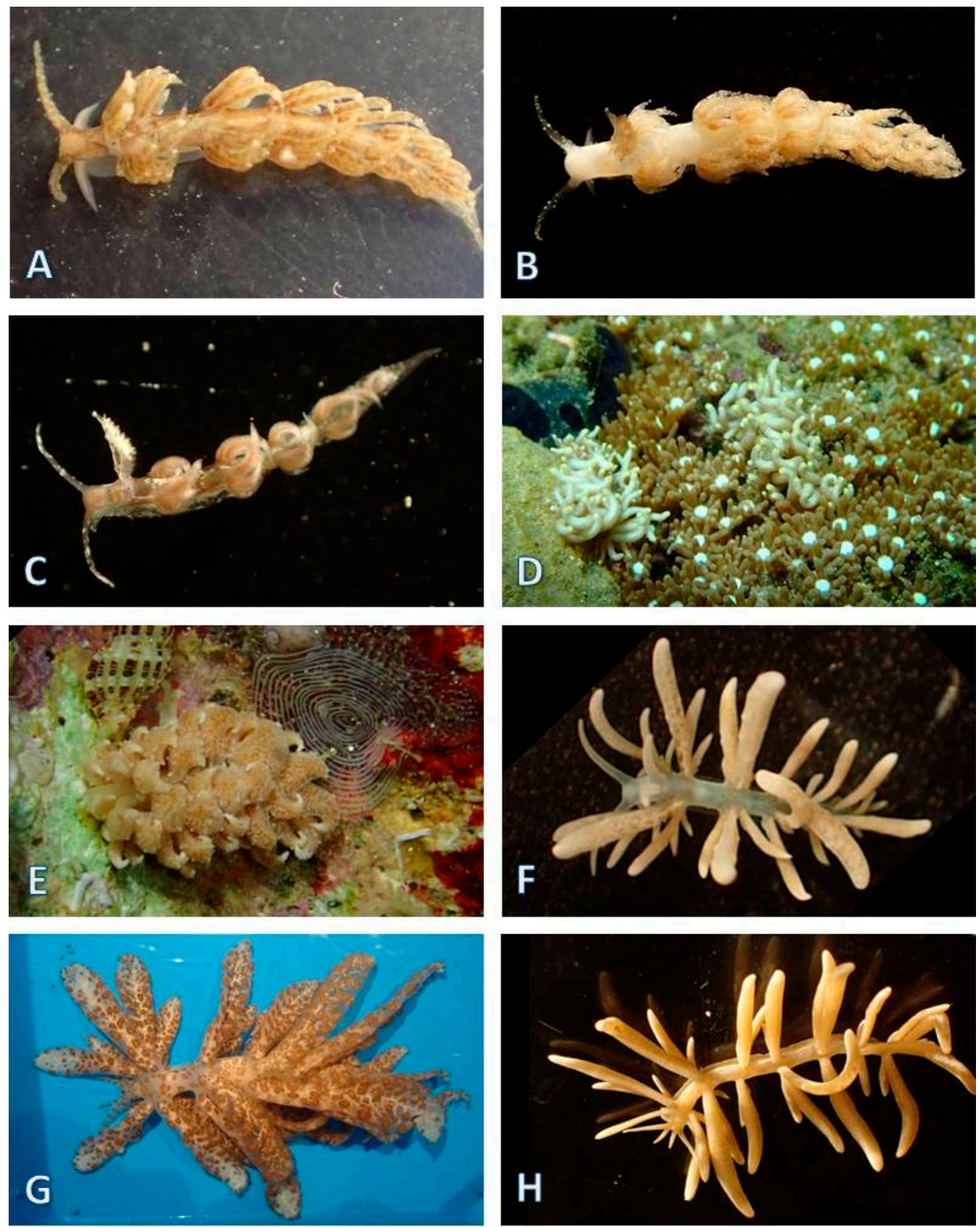

Figure 23. Nudibranchia Cladobranchia: (A) Noumeaella sp. (Noumeaella sp. 2 in Gosliner et al. [24]: 367), Nosp2_17Ba-1; (B) Noumeaella sp. (Noumeaella sp. 3 in Gosliner et al. [24]: 367), Nosp3Ba-3; (C) Noumeaella sp. (Noumeaella sp. 13 in Gosliner et al. [24]: 369), Nosp13Ba-4; (D) Phyllodesmium briareum, Phbr18Ba-2-16; (E) Phyllodesmium cf. crypticum, Phcr17Ba-4; (F) Phyllodesmium lizardense, Phliz18Ba-3; (G) Phyllodesmium longicirrum, Phlo18Ba-1; (H) Phyllodesmium magnum, Phma18Ba-1. 

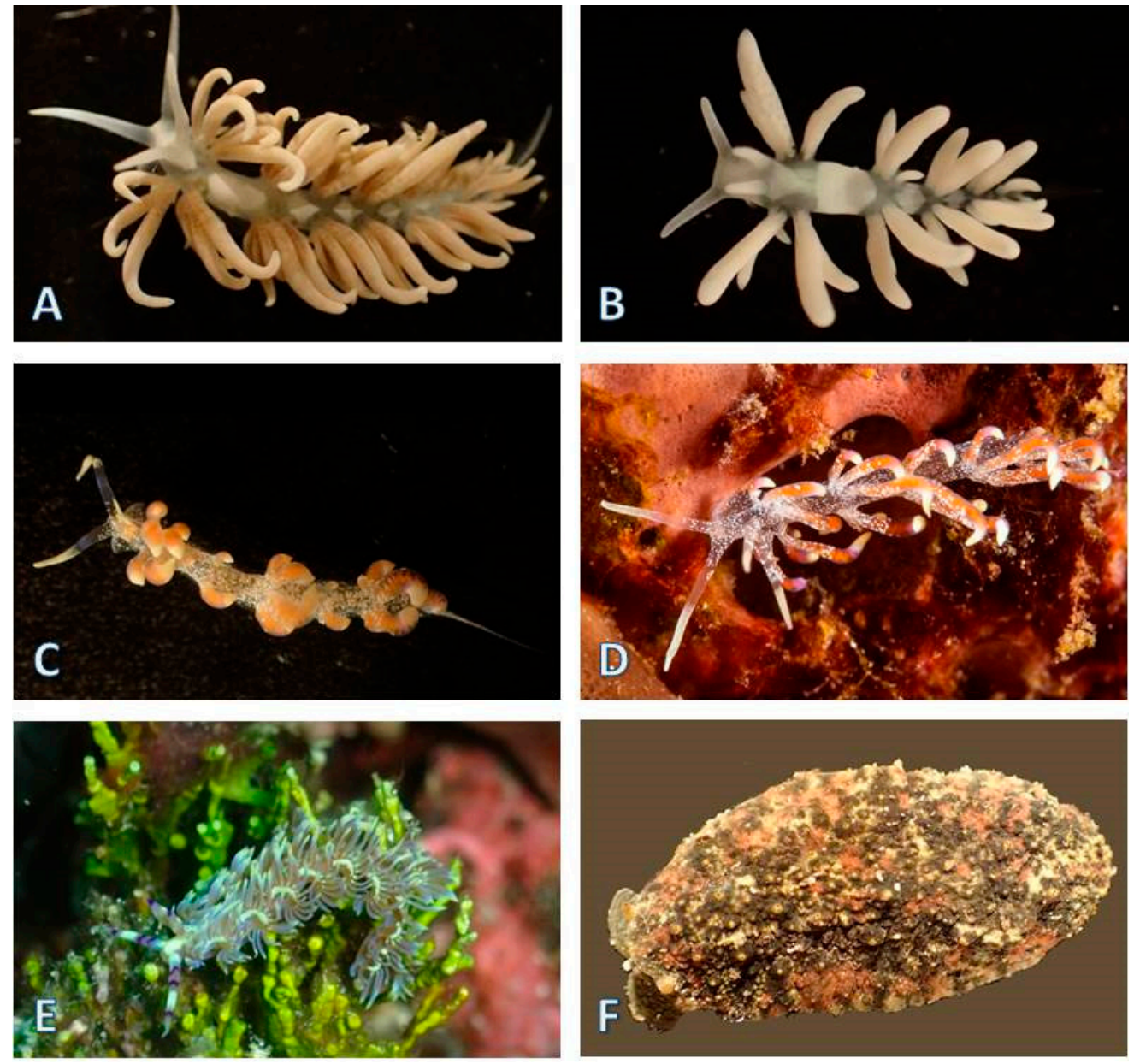

Figure 24. Nudibranchia Cladobranchia and Eupulmonata: (A) Phyllodesmium parangatum, Phpa18Ba-4; (B) Phyllodesmium pecten, Phpe18Ba-1; (C,D*) Phyllodesmium poindimiei, Phpo18Ba-1; (E) Pteraeolidia semperi, Ptse17Ba-2; (F) Peronia sp. a, Onsp18Ba-1.

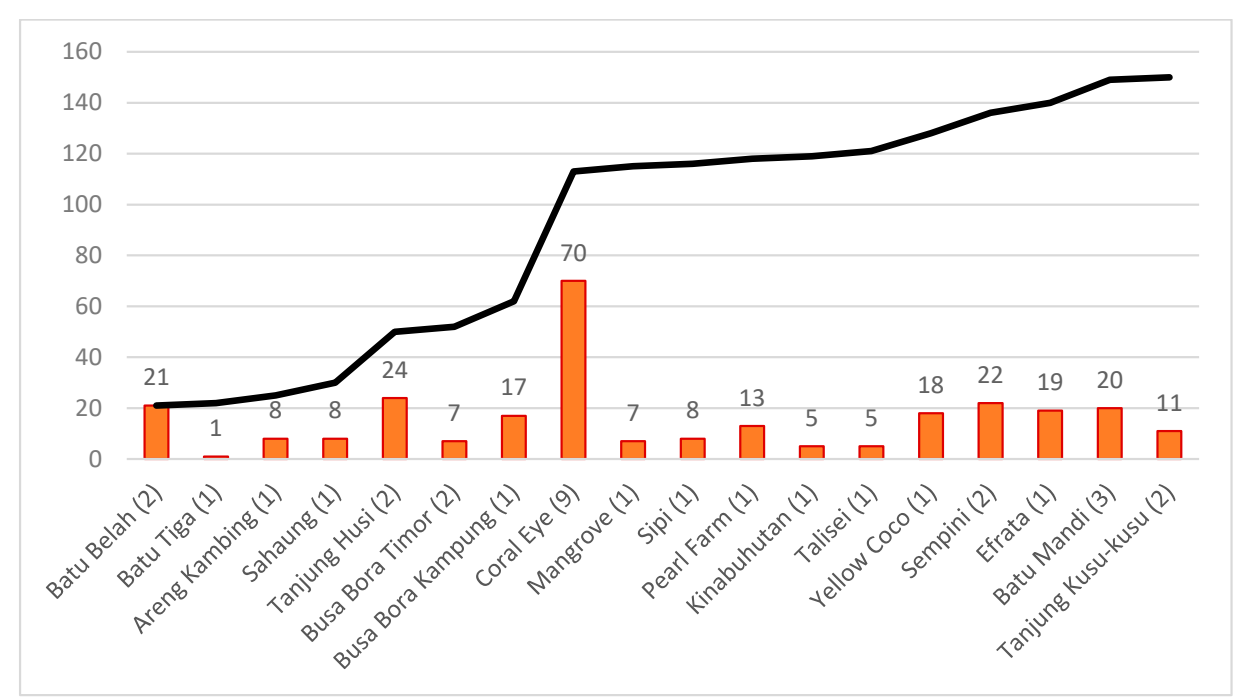

Figure 25. Species accumulation curve (black line) and number of species per locality (red bars). Numbers in brackets after the locality name indicate the number of sampling events at that particular site. Note the high number of sampling events at the locality Coral Eye. 


\section{Discussion}

This study is the first of its kind in the Bangka Archipelago (BA). In total, 484 specimens representing 149 species are recorded for the first time from this area, with a continuous increase in species with every collection area (Figure 25). With 33 undescribed species, more than $20 \%$ of the total species record is not known to science. In comparison to the recent study of Bunaken National Park (BNP) [16], which recorded 69 undescribed species out of a total of 215 species, the percentage of new species in BA is lower. Summarizing our data from Bangka Archipelago and comparing them with previous studies from North Sulawesi (Figure 26), BA represents the second most diverse area with regard to marine heterobranchs. The species accumulation curve also indicates the increase in species around Bangka Archipelago not recorded before. In total, 333 recorded species are now recorded from North Sulawesi.

When comparing higher order levels in the studied regions of North Sulawesi (Figure 27), the distribution of species within the higher taxa is quite similar, with Doridina and Cladobranchia being the most common taxa, followed by Sacoglossa and Cephalaspidea. However, BA shows the highest number of Doridina, whereas Cladobranchia are more common in BNP.

Distribution of specific taxa is highly variable when comparing the different localities in BA (Table 2), with Chromodoris annae (46 individuals) and Phyllidiella pustulosa (39 individuals) dominating the overall collection and being encountered in almost all sites. Unpublished results confirm earlier results from Stoffel et al. [25] and clearly show cryptic speciation in Phyllidiella pustulosa, with sympatric occurrences of the various clades in Bangka Archipelago, BNP, and the island of Sangihe. If these clades are considered separate species, the diversity would increase by five to seven species and thus render P. pustulosa a much less common species. All available $C$. annae specimens from this study and the prior studies seem to cluster in one clade as one species (unpublished data). Other species were also found in high numbers, but not at many sites. A large population of Pteraeolidia semperi was found in one place (Sempini) with more than 100 specimens recorded, probably coming together for mating. More than 50 specimens of Phyllodesmium briareum were found on a single large soft coral colony of Briareum, which extended nearly $2 \mathrm{~m}^{2}$. However, these larger aggregations are unusual. Many species (62) were found with only one individual.

With regard to animals encountered during diving, the family Phyllidiidae seems to be the most dominant one, with members found at almost all dive sites. The animals feed on sponges, on which they sometimes leave a scar (Figure 17G,H). This was already noted before [37,38] where, e.g., Yasman [37] interpreted the scars as an effect of extra-oral digestion.

A few genera are represented by a higher number of species than in other areas of North Sulawesi. Eight species of Phyllodesmium are now recorded from BA; this number is thus much higher than that recorded in BNP, with only four species. Members of Phyllodesmium feed on octocorals, which are very common in our study area.

Recording diversity can be biased by collection efforts (Figure 25). The highest number of species was collected in front of Coral Eye, due to the proximity of this site to the marine laboratory station; this enabled several night dives and also a more thorough search in the coral rubble, which was collected and examined in the laboratory. Coral rubble is an important habitat for smaller heterobranchs, providing a wide array of food items. Specific searches in this habitat yielded many species not found while diving, e.g., the rather cryptic Melibe bucephala, several Gymnodoris spp., Dermatobranchus rodmani, and Dermatobranchus species. 


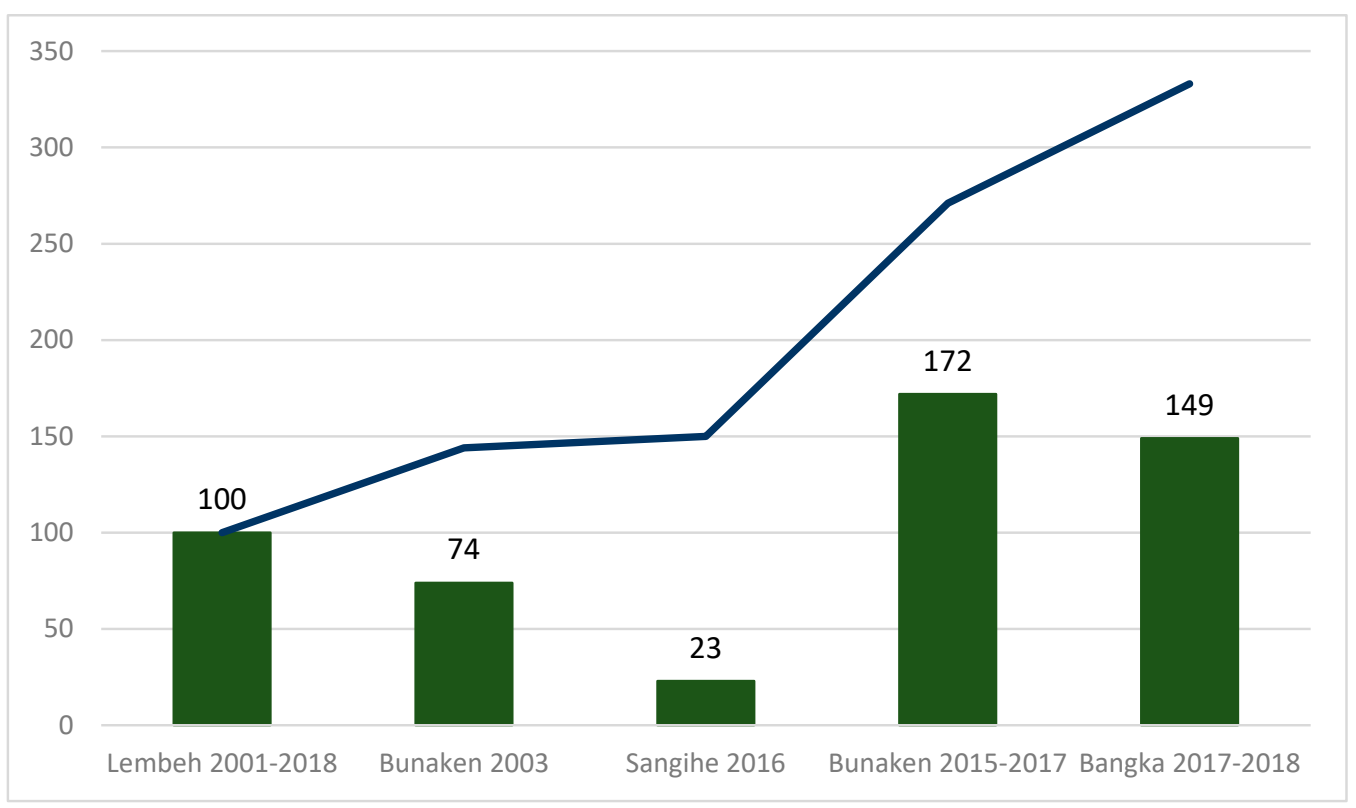

Figure 26. Species accumulation curve and number of species per locality based on the available studies from Lembeh Strait $[17,21,39]$ with the earliest information on diversity from North Sulawesi, Bunaken National Park (BNP) [1,15,16], and Sangihe Island [18].

The lowest number of species and specimens occurred in mangrove areas (close to Sipi and Kinabuhutan). This is certainly because of lower food availability. However, a species typical of this habitat, Jorunna funebris, was recorded from the locality in high numbers (but not all were collected). The record of only one species from Batu Tiga is certainly due to the difficult diving situation on that particular day with strong currents. The locality is dominated by a coral sand flat at a depth of ca. 20 $\mathrm{m}$ with a few coral pinnacles. Snorkeling in front of the destroyed area at the mining site close to Sipi did not result in any records of nudibranchs, due to the still rather fresh stone blocks dumped in front of the site, where only a few corals and sponges were observed to have begun to colonize them. We did not include the mining site in our list of localities, because we did not perform any scuba diving at this particular locality.

Table 3 and Figure 28 list all recorded marine Heterobranchia species from North Sulawesi including our data from Bangka Archipelago (BA) and those published from the three other study areas: Bunaken National Park (BNP) [1,15,16], Sangihe Island (SA) [18], and Lembeh Strait (LS) [17]. Lembeh Strait is particularly famous for its richness in marine heterobranchs, although detailed studies are still lacking and most of the data are only available on the internet. For better comparison, we extracted additional information from Tonozuka [39] and especially from seaslugforum.net [21]. Photographic records provided by many scientists and citizen scientists on this website were evaluated by Bill Rudman and the scientific community in the past. Using information from citizen scientists has become more important lately in documenting changes in species composition [13,40-42]. This is a state-of-the-art species list and enables future monitoring to assess earlier data from this locality. In the following section, we highlight some results from this comparison. 


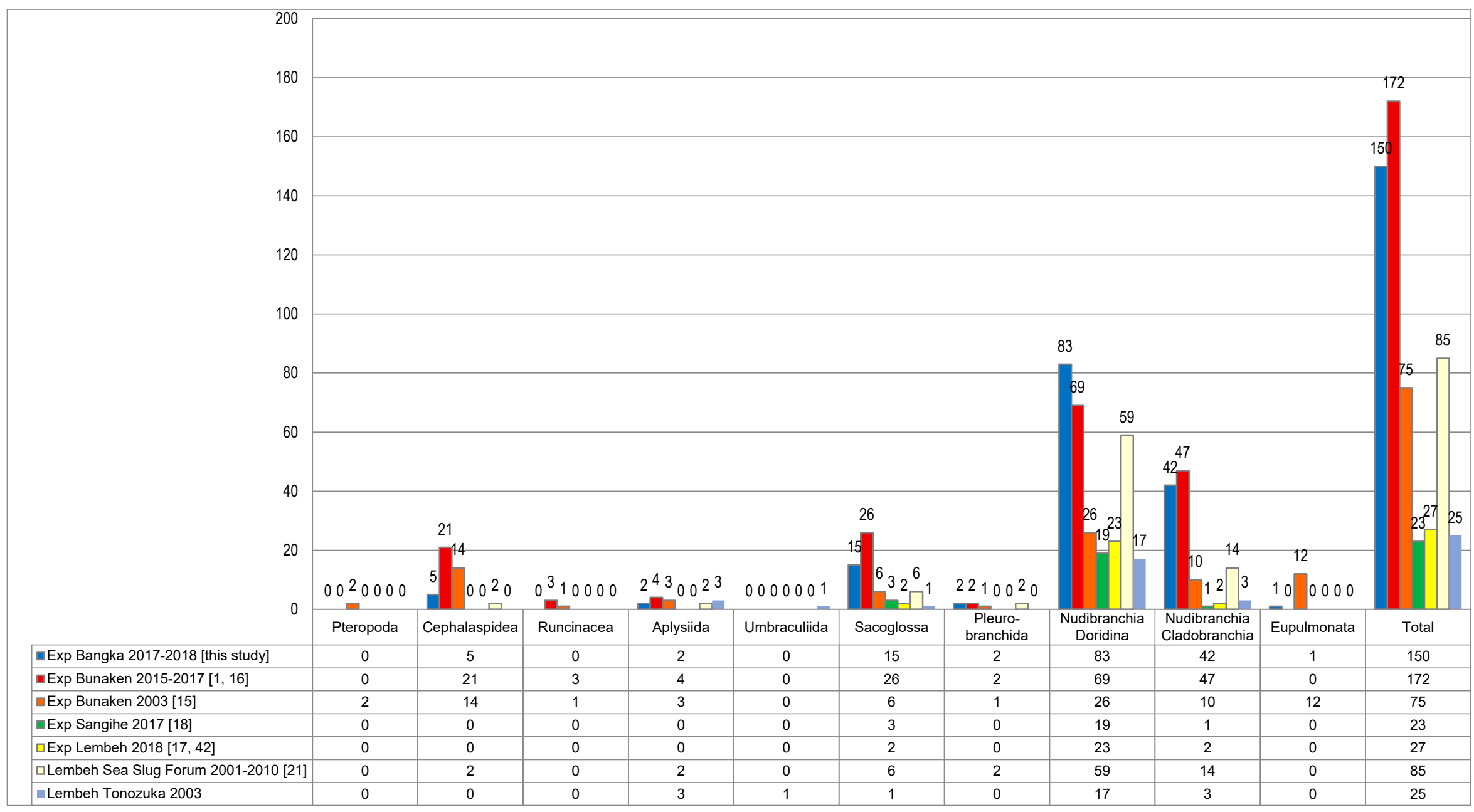

Figure 27. Higher taxa diversity of marine Heterobranchia in Bangka Archipelago compared with other areas in North Sulawesi. Considered are the expeditions to Bunaken National Park [1,15,16], to Sangihe Island [18], and to Lembeh Strait [17,39]. Additional information is accumulated for Lembeh Strait from Sea Slug Forum [21]. Numbers above columns indicate species numbers. 
Table 3. Compilation of the species recorded in this study and those recently published from Bunaken National Park [16], a previous study from Bunaken National Park [15], from Sangihe Island [18], and three sources on Lembeh Strait: Ompi et al. (2019) [17], species records from Lembeh Strait available from the seaslugforum.net [21] in the time period of 2001-2010, and data extracted from Tonozuka [39]. (*) indicate species that were identified only by photo-documentation.

\begin{tabular}{|c|c|c|c|c|c|c|c|c|c|}
\hline \multicolumn{10}{|c|}{ Expedition in Bangka Archipelago and Adjacent Islands } \\
\hline Higher Taxon Affiliation & Identifier & Species Name & $\begin{array}{c}\text { Exp } \\
\text { Bangka } \\
\text { 2017-2018 }\end{array}$ & $\begin{array}{l}\text { Exp Bunaken } \\
2015-2017\end{array}$ & $\begin{array}{l}\text { Exp } \\
\text { Bunaken } \\
2003\end{array}$ & $\begin{array}{l}\text { Exp } \\
\text { Sangihe } \\
2016\end{array}$ & $\begin{array}{l}\text { Exp } \\
\text { Lembeh } \\
2018\end{array}$ & $\begin{array}{l}\text { Lembeh } \\
\text { Sea Slug } \\
\text { Forum } \\
2001-2010\end{array}$ & $\begin{array}{c}\text { Tonozuka } \\
2003\end{array}$ \\
\hline \multicolumn{10}{|c|}{ Pteropoda } \\
\hline Limacinidae Gray, 1840 & & Limacina cf. helicina (Phipps, 1774) & & & $\mathrm{x}$ & & & & \\
\hline $\begin{array}{l}\text { Cavoliniidae Gray, } 1850 \\
\text { (1815) }\end{array}$ & & Cavolinia globulosa Gray, 1850 & & & $\mathrm{x}$ & & & & \\
\hline \multicolumn{10}{|c|}{ Cephalaspidea } \\
\hline Bullidae Lamarck, 1801 & & Bulla cf. ampulla Linnaeus, 1758 & & & $x$ & & & & \\
\hline \multirow{11}{*}{$\begin{array}{l}\text { Haminoeidae Pilsbry, } \\
1895\end{array}$} & & Atys cf. semistriata Pease, 1860 & & & $x$ & & & & \\
\hline & & Haminoea curta (Adams, 1850) & & & $x$ & & & & \\
\hline & $\begin{array}{l}\text { Hasp15Bu-1, } \\
\text { Hasp16Bu-1 }\end{array}$ & $\begin{array}{l}\text { Haminoea sp. } \\
\text { (Haminoea sp. } 2 \text { in Gosliner } \\
\text { et al. [24]: } 30)\end{array}$ & & $x$ & $x$ & & & & \\
\hline & & $\begin{array}{l}\text { Haminoeid sp. } \\
\text { (Haminoeid sp. } 2 \text { in Gosliner } \\
\text { et al. [24]: } 34 \text { ) }\end{array}$ & & $x$ & & & & & \\
\hline & Hasp18Ba-1 & $\begin{array}{l}\text { Haminoea sp. (Haminoea sp. } 3 \\
\text { in Gosliner et al. [24]: 30) }\end{array}$ & $\mathrm{x}$ & & & & & & \\
\hline & Hasp2_15Bu-1 & Haminoea sp. & & $x$ & & & & & \\
\hline & Hasp2_16Bu-1 & Haminoea sp. & & $x$ & & & & & \\
\hline & & Limulatys cf. ooformis Habe, 1952 & & & $x$ & & & & \\
\hline & & $\begin{array}{l}\text { Phanerophthalmus cf. albocollaris } \\
\text { Heller and Thompson, } 1983\end{array}$ & & $\mathrm{X}$ & $\mathrm{x}$ & & & & \\
\hline & & $\begin{array}{l}\text { Phanerophthalmus olivaceus } \\
\text { (Ehrenberg, 1828) as P. smaragdinus }\end{array}$ & & & $x$ & & & & \\
\hline & & $\begin{array}{c}\text { Phanerophthalmus sp. } \\
\text { (Phanerophthalmus sp. } 3 \text { in Gosliner } \\
\text { et al. [24]: } 33 \text { ) }\end{array}$ & & $x$ & & & & & \\
\hline
\end{tabular}


Table 3. Cont

\begin{tabular}{|c|c|c|c|c|c|c|c|c|c|}
\hline \multicolumn{10}{|c|}{ Expedition in Bangka Archipelago and Adjacent Islands } \\
\hline Higher Taxon Affiliation & Identifier & Species Name & $\begin{array}{c}\text { Exp } \\
\text { Bangka } \\
\text { 2017-2018 }\end{array}$ & $\begin{array}{l}\text { Exp Bunaken } \\
\text { 2015-2017 }\end{array}$ & $\begin{array}{c}\text { Exp } \\
\text { Bunaken } \\
2003\end{array}$ & $\begin{array}{c}\text { Exp } \\
\text { Sangihe } \\
2016\end{array}$ & $\begin{array}{l}\text { Exp } \\
\text { Lembeh } \\
2018\end{array}$ & $\begin{array}{l}\text { Lembeh } \\
\text { Sea Slug } \\
\text { Forum } \\
2001-2010\end{array}$ & $\begin{array}{c}\text { Tonozuka } \\
2003\end{array}$ \\
\hline $\begin{array}{l}\text { Philinidae Gray, } 1850 \\
\text { (1815) }\end{array}$ & Ilsp17Bu-1 & Philine sp. & & $\mathrm{x}$ & $\mathrm{X}$ & & & & \\
\hline \multirow{2}{*}{$\begin{array}{l}\text { Philinoglossidae } \\
\text { Hertling, } 1932\end{array}$} & & Philinoglossa marcusi Challis, 1969 & & & $x$ & & & & \\
\hline & & $\begin{array}{c}\text { Philinoglossa sp. } \\
\text { (in Gosliner et al. [24]: 103) }\end{array}$ & & & $x$ & & & & \\
\hline $\begin{array}{l}\text { Colpodaspididae } \\
\text { Oskars, Bouchet and } \\
\text { Malaquias, } 2015\end{array}$ & & Colpodaspis thompsoni Brown, 1979 & $\mathrm{x}$ & $x$ & $x$ & & & & \\
\hline \multirow{6}{*}{$\begin{array}{c}\text { Aglajidae } \\
\text { Pilsbry, } 1895 \text { (1847) }\end{array}$} & Agsp15Bu-1 & Aglajidae sp. & & $x$ & & & & & \\
\hline & & Chelidonura amoena Bergh, 1905 & $x$ & $x$ & $x$ & & & $x$ & \\
\hline & & $\begin{array}{l}\text { Chelidonura hirundinina } \\
\text { (Quoy and Gaimard, 1833) }\end{array}$ & & $\mathrm{x}$ & $\mathrm{X}$ & & & & \\
\hline & Odsp.a18Ba-1 & $\begin{array}{c}\text { Odontoglaja cf. guamensis Rudman, } \\
1978\end{array}$ & $\mathrm{X}$ & $x$ & & & & & \\
\hline & & Philinopsis speciosa Pease, 1860 & & & & & & $x$ & \\
\hline & Phisp16Bu-1 & Tubulophilinopsis sp. & & $x$ & & & & & \\
\hline \multirow{8}{*}{$\begin{array}{c}\text { Gastropteridae Swainson, } \\
1840\end{array}$} & Gasp5_16Bu-1 & $\begin{array}{l}\text { Gastropteron sp. (Gastropteron sp. } 5 \\
\text { in Gosliner et al. [24]: } 56 \text { ) }\end{array}$ & & $\mathrm{x}$ & & & & & \\
\hline & & $\begin{array}{l}\text { Sagaminopteron psychedelicum } \\
\text { Carlson and Hoff, } 1974\end{array}$ & $\mathrm{x}$ & $x$ & & & & & \\
\hline & Sasp17Bu-1 & Sagaminopteron sp. & & $x$ & & & & & \\
\hline & & $\begin{array}{l}\text { Siphopteron brunneomarginatum } \\
\text { (Carlson and Hoff, 1974) }\end{array}$ & & $x$ & & & & & \\
\hline & & $\begin{array}{c}\text { Siphopteron ladrones } \\
\text { (Carlson and Hoff, 1974) }\end{array}$ & & $x$ & & & & & \\
\hline & & $\begin{array}{l}\text { Siphopteron nigromarginatu } \\
\text { mGosliner, } 1989\end{array}$ & & $x$ & & & & & \\
\hline & & Siphopteron tigrinum Gosliner, 1989 & & $x$ & $x$ & & & & \\
\hline & Sini15Bu-19+20 & Siphopteron $\mathrm{sp.}$ & & $x$ & & & & & \\
\hline
\end{tabular}


Table 3. Cont

\begin{tabular}{|c|c|c|c|c|c|c|c|c|c|}
\hline \multicolumn{10}{|c|}{ Expedition in Bangka Archipelago and Adjacent Islands } \\
\hline Higher Taxon Affiliation & Identifier & Species Name & $\begin{array}{c}\text { Exp } \\
\text { Bangka } \\
\text { 2017-2018 }\end{array}$ & $\begin{array}{l}\text { Exp Bunaken } \\
\text { 2015-2017 }\end{array}$ & $\begin{array}{c}\text { Exp } \\
\text { Bunaken } \\
2003\end{array}$ & $\begin{array}{c}\text { Exp } \\
\text { Sangihe } \\
2016\end{array}$ & $\begin{array}{l}\text { Exp } \\
\text { Lembeh } \\
2018\end{array}$ & $\begin{array}{l}\text { Lembeh } \\
\text { Sea Slug } \\
\text { Forum } \\
2001-2010\end{array}$ & $\begin{array}{c}\text { Tonozuka } \\
2003\end{array}$ \\
\hline \multicolumn{10}{|c|}{ Runcinacea } \\
\hline \multirow{3}{*}{$\begin{array}{c}\text { Runcinidae } \\
\text { H. Adams and A. Adams, } \\
1854\end{array}$} & Rusp15Bu-1 & Runcina sp. & & $x$ & $x$ & & & & \\
\hline & Rusp16Bu-1 & Runcina sp. & & $x$ & & & & & \\
\hline & $\begin{array}{l}\text { Rusp2_16Bu-1 } \\
\text { Rusp3_16Bu-1 }\end{array}$ & Runcina sp. & & $\mathrm{x}$ & & & & & \\
\hline \multicolumn{10}{|c|}{ Aplysiida } \\
\hline & & $\begin{array}{c}\text { Aplysia oculifera } \\
\text { A. Adams and Reeve, } 1850\end{array}$ & & & & & & & $\mathrm{x}$ \\
\hline \multirow{9}{*}{ Aplysiidae Lamarck, 1809} & & Bursatella leachii Blainville, 1817 & & & & & & $x$ & \\
\hline & & $\begin{array}{l}\text { Dolabella auricularia (Lightfoot, } \\
\text { 1786) }\end{array}$ & & & & & & & $x$ \\
\hline & & Petalifera ramosa Baba, 1959 & & & & & & & $x$ \\
\hline & & $\begin{array}{c}\text { Aplysia cf. nigrocincta von Martens, } \\
1880\end{array}$ & $\mathrm{x}$ & $\mathrm{X}$ & & & & & \\
\hline & & $\begin{array}{c}\text { Stylocheilus striatus } \\
\text { (Quoy and Gaimard, 1832) }\end{array}$ & $x$ & $\mathrm{X}$ & $x$ & & & & \\
\hline & & $\begin{array}{l}\text { Dolabella auricularia (Lightfoot, } \\
\text { 1786) }\end{array}$ & & $x$ & $\mathrm{X}$ & & & & \\
\hline & & Dolabrifera dolabrifera (Rang, 1828) & & $x$ & & & & & \\
\hline & & Phyllaplysia sp. & & & $\mathrm{X}$ & & & & \\
\hline & & $\begin{array}{l}\text { Syphonota geographica } \\
\text { (A. Adams and Reeve, 1850) }\end{array}$ & & & & & & $x$ & \\
\hline \multicolumn{10}{|c|}{ Umbraculida } \\
\hline $\begin{array}{l}\text { Umbraculiidae Dall, } 1889 \\
\text { (1827) }\end{array}$ & & $\begin{array}{l}\text { Umbraculum umbraculum } \\
\text { (Lightfoot, 1786) }\end{array}$ & & & & & & & $\mathrm{x}$ \\
\hline
\end{tabular}


Table 3. Cont

\begin{tabular}{|c|c|c|c|c|c|c|c|c|c|}
\hline \multicolumn{10}{|c|}{ Expedition in Bangka Archipelago and Adjacent Islands } \\
\hline Higher Taxon Affiliation & Identifier & Species Name & $\begin{array}{c}\text { Exp } \\
\text { Bangka } \\
\text { 2017-2018 }\end{array}$ & $\begin{array}{l}\text { Exp Bunaken } \\
\text { 2015-2017 }\end{array}$ & $\begin{array}{l}\text { Exp } \\
\text { Bunaken } \\
2003\end{array}$ & $\begin{array}{l}\text { Exp } \\
\text { Sangihe } \\
2016\end{array}$ & $\begin{array}{l}\text { Exp } \\
\text { Lembeh } \\
2018\end{array}$ & $\begin{array}{l}\text { Lembeh } \\
\text { Sea Slug } \\
\text { Forum } \\
\text { 2001-2010 }\end{array}$ & $\begin{array}{c}\text { Tonozuka } \\
2003\end{array}$ \\
\hline \multicolumn{10}{|c|}{ Sacoglossa } \\
\hline $\begin{array}{c}\text { Cylindrobullidae Thiele, } \\
1931\end{array}$ & Assp1_17Bu-1-4 & Cylindrobulla sp. & & $x$ & & & & & \\
\hline \multirow[b]{2}{*}{$\begin{array}{c}\text { Oxynoidae } \\
\text { Stoliczka, } 1868 \text { (1847) }\end{array}$} & & Lobiger nevilli Pilsbry, 1896 & & $x$ & & & & & \\
\hline & $\begin{array}{l}\text { Losp1-18Ba1; } \\
\text { Losp1-18Ba2 }\end{array}$ & $\begin{array}{l}\text { Lobiger sp. } \\
\text { (Lobiger sp. } 1 \text { in Gosliner } \\
\text { et al. [24]: 70) }\end{array}$ & $\mathrm{x}$ & $\mathrm{x}$ & & & & & \\
\hline \multirow{6}{*}{$\begin{array}{l}\text { Hermaeidae H. Adams } \\
\text { and A. Adams, } 1854\end{array}$} & & Cyerce bourbonica Yonow, 2012 & $\mathrm{x}$ & $\mathrm{x}$ & $\begin{array}{c}\mathrm{X} \\
\text { as C. sp. } 1\end{array}$ & & & & \\
\hline & & Cyerce elegans Bergh, 1870 & & & $x$ & & & & \\
\hline & & Cyerce nigra Bergh, 1871 & $\mathrm{x}$ & & & & & & \\
\hline & Cysp2_15Bu-5 & Cyerce sp. & & $\mathrm{x}$ & & & & & \\
\hline & & $\begin{array}{l}\text { Hermaea sp. (Hermaea sp. } 2 \text { in } \\
\text { Gosliner et al. [24]: 81); Aplysiopsis } \\
\text { sp. } 1 \text { in Sea Slug Forum) }\end{array}$ & & & & & & $\mathrm{x}$ & \\
\hline & & Sohgenia palauensis Hamatani, 1991 & & $x$ & & & & & \\
\hline \multirow{4}{*}{$\begin{array}{c}\text { Costasiellidae Clarke, } \\
1984\end{array}$} & Cosp17Bu-1 & $\begin{array}{l}\text { Costasiella kuroshimae } \\
\text { Ichikawa, } 1993\end{array}$ & & $x$ & & & & $\begin{array}{l}\text { Xas } \\
\text { Chromodoris } \\
\text { sp. } 3\end{array}$ & \\
\hline & Cosp1_17Bu-1-2 & $\begin{array}{l}\text { Costasiella sp. (Costasiella sp. } 1 \text { in } \\
\text { Gosliner et al. [24]: 79) }\end{array}$ & & $\mathrm{x}$ & & & & & \\
\hline & Cosp8_17Bu-1 & $\begin{array}{l}\text { Costasiella sp. (Costasiella sp. } 8 \text { in } \\
\text { Gosliner et al. [24]: } 81 \text { ) }\end{array}$ & & $\mathrm{x}$ & & & & & \\
\hline & Cosp3_16Bu-1-5 & Costasiella sp. & & $x$ & & & & & \\
\hline
\end{tabular}


Table 3. Cont

\begin{tabular}{|c|c|c|c|c|c|c|c|c|c|}
\hline \multicolumn{10}{|c|}{ Expedition in Bangka Archipelago and Adjacent Islands } \\
\hline Higher Taxon Affiliation & Identifier & Species Name & $\begin{array}{c}\text { Exp } \\
\text { Bangka } \\
\text { 2017-2018 }\end{array}$ & $\begin{array}{l}\text { Exp Bunaken } \\
\text { 2015-2017 }\end{array}$ & $\begin{array}{c}\text { Exp } \\
\text { Bunaken } \\
2003\end{array}$ & $\begin{array}{l}\text { Exp } \\
\text { Sangihe } \\
2016\end{array}$ & $\begin{array}{c}\text { Exp } \\
\text { Lembeh } \\
2018\end{array}$ & $\begin{array}{l}\text { Lembeh } \\
\text { Sea Slug } \\
\text { Forum } \\
\text { 2001-2010 }\end{array}$ & $\begin{array}{c}\text { Tonozuka } \\
2003\end{array}$ \\
\hline \multirow{17}{*}{$\begin{array}{l}\text { Plakobranchidae Gray, } \\
\qquad 1840\end{array}$} & & $\begin{array}{l}\text { Elysia asbecki Wägele, Stemmer, } \\
\text { Burghardt and Händeler, } 2010\end{array}$ & $\mathrm{x}$ & $\mathrm{x}$ & & & & & \\
\hline & & Elysia grandifolia Kelaart, 1858 & & & & & & $x$ & \\
\hline & & Elysia marginata (Pease, 1871) & $x$ & $x$ & & & & & \\
\hline & & Elysia mercieri (Pruvot-Fol, 1930) & & $\mathrm{x}$ & & & & & \\
\hline & & Elysia cf nigropunctata (Pease, 1871) & $\mathrm{x}$ & & & & & & \\
\hline & & Elysia ornata (Swainson, 1840) & & & & & & $x$ & \\
\hline & & Elysia pusilla (Bergh, 1871) & $x$ & $x$ & $x$ & $x$ & & & \\
\hline & Elsp.a18Ba-1 & Elysia sp. (Elysia sp. a) & $x$ & $x$ & & & & & \\
\hline & Elsp.b18Ba-1 & Elysia sp. (Elysia sp. b) & $x$ & $x$ & & & & & \\
\hline & Elsp1_16Bu-1 & Elysia sp. & & $x$ & & & & & \\
\hline & Elsp16Bu-1 & Elysia sp. & & $x$ & & & & & \\
\hline & Elsp4_16Bu-1 & Elysia sp. & & $x$ & & & & & \\
\hline & Elsp24-18Ba-1 & $\begin{array}{l}\text { Elysia sp. } 24 \text { (in Gosliner et al. [24]: } \\
\text { 89) }\end{array}$ & $x$ & & & & & & \\
\hline & Elsp27-18Ba-1 & $\begin{array}{l}\text { Elysia sp. } 27 \text { (in Gosliner et al. [24]: } \\
\text { 89) }\end{array}$ & $x$ & & & & & & \\
\hline & & $\begin{array}{c}\text { Plakobranchus ocellatus van Hasselt, } \\
1824\end{array}$ & & $x$ & & & & & \\
\hline & & $\begin{array}{l}\text { Plakobranchus cf. papua } \\
\text { Meyers-Muños and van der Velde, } \\
2016\end{array}$ & & & & $x$ & & & \\
\hline & & $\begin{array}{c}\text { Thuridilla albopustulosa Gosliner, } \\
1995\end{array}$ & & $x$ & & & & $x$ & \\
\hline
\end{tabular}


Table 3. Cont

\begin{tabular}{|c|c|c|c|c|c|c|c|c|c|}
\hline \multicolumn{10}{|c|}{ Expedition in Bangka Archipelago and Adjacent Islands } \\
\hline Higher Taxon Affiliation & Identifier & Species Name & $\begin{array}{c}\text { Exp } \\
\text { Bangka } \\
\text { 2017-2018 }\end{array}$ & $\begin{array}{c}\text { Exp Bunaken } \\
2015-2017\end{array}$ & $\begin{array}{c}\text { Exp } \\
\text { Bunaken } \\
2003\end{array}$ & $\begin{array}{c}\text { Exp } \\
\text { Sangihe } \\
2016\end{array}$ & $\begin{array}{c}\text { Exp } \\
\text { Lembeh } \\
2018\end{array}$ & $\begin{array}{l}\text { Lembeh } \\
\text { Sea Slug } \\
\text { Forum } \\
\text { 2001-2010 }\end{array}$ & $\begin{array}{c}\text { Tonozuka } \\
2003\end{array}$ \\
\hline & & Thuridilla carlsoni Gosliner, 1995 & $\mathrm{x}$ & & & & & & \\
\hline & & $\begin{array}{l}\text { Thuridilla flavomaculata Gosliner, } \\
1995\end{array}$ & $x$ & $x$ & & & & & \\
\hline & & Thuridilla vataae (Risbec, 1928) & $x$ & $x$ & & & & & \\
\hline & & Thuridilla gracilis (Risbec, 1928) & $x$ & $\mathrm{x}$ & $\begin{array}{c}\mathrm{X} \\
\text { as T.bayeri }\end{array}$ & $x$ & & $x$ & \\
\hline & & Thuridilla cf. hoffae Gosliner, 1995 & & & $x$ & & & & \\
\hline & & Thuridilla lineolate (Bergh, 1905) & & $x$ & $x$ & & & & $x$ \\
\hline & & Thuridilla livida (Baba, 1955) & & $\mathrm{x}$ & & & & & \\
\hline & & Thuridilla undula Gosliner, 1995 & & $x$ & & & & & \\
\hline \multicolumn{10}{|c|}{ Pleurobranchida } \\
\hline \multirow{4}{*}{$\begin{array}{c}\text { Pleurobranchidae Gray, } \\
1827\end{array}$} & & Berthellina citrina (Pease, 1861) & & $x$ & & & & & \\
\hline & & Berthella martensi (Pilsbry, 1896) & & & & & & $x$ & \\
\hline & & $\begin{array}{c}\text { Pleurobranchus forskalii } \\
\text { Rüppell and Leuckart, } 1828\end{array}$ & $x$ & $x$ & & & & & \\
\hline & & Pleurobranchus peronii Cuvier, 1804 & $x$ & & $x$ & & & $x$ & \\
\hline \multicolumn{10}{|c|}{ Nudibranchia, Doridina } \\
\hline $\begin{array}{c}\text { Hexabranchidae Bergh, } \\
1891\end{array}$ & Glsp1_17Ba-1 & $\begin{array}{l}\text { Hexabranchus sanguineus (Rüppell } \\
\text { and Leuckart, 1830) }\end{array}$ & $x$ & $x$ & $x$ & & & & \\
\hline
\end{tabular}


Table 3. Cont.

\begin{tabular}{|c|c|c|c|c|c|c|c|c|c|}
\hline \multicolumn{10}{|c|}{ Expedition in Bangka Archipelago and Adjacent Islands } \\
\hline Higher Taxon Affiliation & Identifier & Species Name & $\begin{array}{c}\text { Exp } \\
\text { Bangka } \\
\text { 2017-2018 }\end{array}$ & $\begin{array}{c}\text { Exp Bunaken } \\
\text { 2015-2017 }\end{array}$ & $\begin{array}{c}\text { Exp } \\
\text { Bunaken } \\
2003\end{array}$ & $\begin{array}{c}\text { Exp } \\
\text { Sangihe } \\
2016\end{array}$ & $\begin{array}{c}\text { Exp } \\
\text { Lembeh } \\
2018\end{array}$ & $\begin{array}{l}\text { Lembeh } \\
\text { Sea Slug } \\
\text { Forum } \\
\text { 2001-2010 }\end{array}$ & $\begin{array}{l}\text { Tonozuka } \\
2003\end{array}$ \\
\hline \multirow{16}{*}{$\begin{array}{l}\text { Polyceridae Alder and } \\
\text { Hancock, } 1845\end{array}$} & & $\begin{array}{c}\text { Kaloplocamus dokte } \\
\text { Vallès and Gosliner, } 2006\end{array}$ & & $\mathrm{x}$ & & & & & \\
\hline & Kalsp8_16Bu-1 & $\begin{array}{l}\text { Kaloplocamus sp. (Kaloplocamus sp. } \\
8 \text { in Gosliner et al. [24]: 116) }\end{array}$ & & $\mathrm{x}$ & & & & & \\
\hline & Kalsp9_16Bu-1 & $\begin{array}{l}\text { Kaloplocamus sp. (Kaloplocamus sp. } \\
9 \text { in Gosliner et al. [24]: 116) }\end{array}$ & & $\mathrm{x}$ & & & & & \\
\hline & & $\begin{array}{l}\text { Nembrotha chamberlaini } \\
\text { Gosliner and Behrens, } 1997\end{array}$ & $\mathrm{x}$ & & & & & & \\
\hline & & Nembrotha cristata Bergh, 1877 & $\mathrm{x}$ & $\mathrm{x}$ & & & & & \\
\hline & & Nembrotha kubaryana Bergh, 1877 & $\mathrm{x}$ & $\mathrm{x}$ & & & $\mathrm{x}$ & $x$ & \\
\hline & & Nembrotha lineolata Bergh, 1905 & $\mathrm{x}$ & & & & & & \\
\hline & & Nembrotha megalocera Yonow, 1990 & & & & & & & $\begin{array}{c}\mathrm{X} \\
\text { misidentified? }\end{array}$ \\
\hline & & $\begin{array}{c}\text { Nembrotha milleri } \\
\text { Gosliner and Behrens, } 1997\end{array}$ & $\mathrm{x}$ & & & & & & \\
\hline & & $\begin{array}{c}\text { Nembrotha mullineri } \\
\text { Gosliner and Behrens, } 1997\end{array}$ & $\mathrm{x}$ & & & & & & \\
\hline & & $\begin{array}{l}\text { Nembrotha purpureolineata } \\
\text { O'Donoghue, } 1924\end{array}$ & & & & & & $\begin{array}{c}\mathrm{X} \\
\text { as N. rutilans }\end{array}$ & \\
\hline & Nesp1_17Ba-1 & $\begin{array}{c}\text { Nembrotha sp. } 1 \\
\text { (in Gosliner et al. [24]: 122) }\end{array}$ & $\mathrm{x}$ & & & & & & \\
\hline & & Polycera japonica Baba, 1949 & & $\mathrm{x}$ & & & & & \\
\hline & & Polycera risbeci Odhner, 1941 & & $\mathrm{x}$ & & & & & \\
\hline & Posp516Bu-1 & $\begin{array}{l}\text { Polycera sp. (Polycera sp. } 5 \text { in } \\
\text { Gosliner et al. [24]: 113) }\end{array}$ & & $\mathrm{x}$ & & & & & \\
\hline & & Roboastra gracilis (Bergh, 1877) & & $\mathrm{x}$ & $x$ & & & & \\
\hline
\end{tabular}


Table 3. Cont

\begin{tabular}{|c|c|c|c|c|c|c|c|c|c|}
\hline \multicolumn{10}{|c|}{ Expedition in Bangka Archipelago and Adjacent Islands } \\
\hline Higher Taxon Affiliation & Identifier & Species Name & $\begin{array}{c}\text { Exp } \\
\text { Bangka } \\
\text { 2017-2018 }\end{array}$ & $\begin{array}{c}\text { Exp Bunaken } \\
2015-2017\end{array}$ & $\begin{array}{c}\text { Exp } \\
\text { Bunaken } \\
2003\end{array}$ & $\begin{array}{c}\text { Exp } \\
\text { Sangihe } \\
2016\end{array}$ & $\begin{array}{c}\text { Exp } \\
\text { Lembeh } \\
2018\end{array}$ & $\begin{array}{l}\text { Lembeh } \\
\text { Sea Slug } \\
\text { Forum } \\
2001-2010\end{array}$ & $\begin{array}{c}\text { Tonozuka } \\
2003\end{array}$ \\
\hline & & $\begin{array}{c}\text { Tambja gabrielae } \\
\text { Pola, Cervera and Gosliner, } 2005\end{array}$ & $x$ & & & & & & $\mathrm{x}$ \\
\hline & & Tambja morosa (Bergh, 1877) & $x$ & & $\mathrm{x}$ & & & & \\
\hline & & Thecacera picta Baba, 1972 & & & & & & & $\mathrm{x}$ \\
\hline & & Gymnodoris aurita (Gould, 1852) * & $x$ & & & & & & \\
\hline & & Gymnodoris citrina (Bergh, 1877) & & & $\mathrm{x}$ & & & & \\
\hline & Gysp2_16Bu-1 & $\begin{array}{l}\text { Gymnodoris sp. (Gymnodoris sp. } 2 \\
\text { in Gosliner et al. [24]: 152) }\end{array}$ & & $x$ & & & & & \\
\hline & Gysp16Bu-1 & $\begin{array}{l}\text { Gymnodoris sp. (Gymnodoris cf. sp. } \\
35 \text { in Gosliner et al. [24]: 159) }\end{array}$ & & $\mathrm{x}$ & & & & & \\
\hline & $\begin{array}{l}\text { Gysp1_17Bu-1 } \\
\text { Gysp22_17Bu-1 }\end{array}$ & $\begin{array}{l}\text { Gymnodoris sp. (Gymnodoris cf. sp. } \\
46 \text { in Gosliner et al. [24]: 161) }\end{array}$ & & $\mathrm{x}$ & & & & & \\
\hline & Gysp1_15Bu-2 & Gymnodoris sp. & & $x$ & & & & & \\
\hline & & $\begin{array}{c}\text { Gymnodoris tuberculosa } \\
\text { Knutson and Gosliner, } 2014\end{array}$ & $x$ & $x$ & & & & & \\
\hline & & Thecacera sp. & & & & & & $x$ & \\
\hline & Gysp20-18Ba-1 & $\begin{array}{c}\text { Gymnodoris sp. 20 } \\
\text { (in Gosliner et al. [24]: 156) }\end{array}$ & $x$ & & & & & & \\
\hline & Gysp25-18Ba-1-2 & $\begin{array}{c}\text { Gymnodoris sp. 25 } \\
\text { (in Gosliner et al. [24]: 157) }\end{array}$ & $x$ & & & & & & \\
\hline
\end{tabular}


Table 3. Cont

\begin{tabular}{|c|c|c|c|c|c|c|c|c|c|}
\hline \multicolumn{10}{|c|}{ Expedition in Bangka Archipelago and Adjacent Islands } \\
\hline Higher Taxon Affiliation & Identifier & Species Name & $\begin{array}{c}\text { Exp } \\
\text { Bangka } \\
\text { 2017-2018 }\end{array}$ & $\begin{array}{l}\text { Exp Bunaken } \\
\text { 2015-2017 }\end{array}$ & $\begin{array}{c}\text { Exp } \\
\text { Bunaken } \\
2003\end{array}$ & $\begin{array}{l}\text { Exp } \\
\text { Sangihe } \\
2016\end{array}$ & $\begin{array}{l}\text { Exp } \\
\text { Lembeh } \\
2018\end{array}$ & $\begin{array}{l}\text { Lembeh } \\
\text { Sea Slug } \\
\text { Forum } \\
2001-2010\end{array}$ & $\begin{array}{c}\text { Tonozuka } \\
2003\end{array}$ \\
\hline \multirow{6}{*}{$\begin{array}{c}\text { Goniodorididae } \\
\text { H. Adams and A. Adams, } \\
1854\end{array}$} & Gosp7-18Ba-1 & $\begin{array}{c}\text { Goniodoris sp. } 7 \\
\text { (in Gosliner et al. [24]: 153) }\end{array}$ & $x$ & & & & & & \\
\hline & & Okenia kendi Gosliner, 2004 & & & & & & $x$ & \\
\hline & & Okenia pellucida Burn, 1967 & & & & & & $x$ & \\
\hline & & $\begin{array}{c}\text { Trapania armilla } \\
\text { Gosliner and Fahey, } 2008\end{array}$ & $x$ & & & & & $x$ & \\
\hline & & $\begin{array}{c}\text { Trapania euryeia } \\
\text { Gosliner and Fahey, } 2008\end{array}$ & & $x$ & & & & & \\
\hline & & Trapania safracornia Fahey, 2004 & $x$ & & & & & & \\
\hline \multirow{4}{*}{ Aegiridae P. Fischer, 1883} & & Aegires citrinus Pruvot-Fol, 1930 & & $x$ & & & & & \\
\hline & Aesp7-18Ba-1 & $\begin{array}{c}\text { Aegires sp. } 7 \\
\text { (in Gosliner et al. [24]: 149) }\end{array}$ & $x$ & & & & & & \\
\hline & & Notodoris minor Eliot, 1904 * & $x$ & & & & & & $x$ \\
\hline & & $\begin{array}{c}\text { Notodoris serenae } \\
\text { Gosliner and Behrens, } 1997\end{array}$ & & $x$ & & $x$ & & & \\
\hline \multirow{2}{*}{$\begin{array}{l}\text { Doridoidea Rafinesque, } \\
1815\end{array}$} & Dosp17Bu-3 & Doridoidea sp. & & $x$ & & & & & \\
\hline & Scsp1_17Bu-1 & Doridoidea sp. & & $x$ & & & & & \\
\hline Cadlinidae Bergh, 1891 & & $\begin{array}{l}\text { Aldisa albatrossae } \\
\text { Elwood, Valdés and Gosliner, } 2000\end{array}$ & & & & & & $x$ & \\
\hline
\end{tabular}


Table 3. Cont

\begin{tabular}{|c|c|c|c|c|c|c|c|c|c|}
\hline \multicolumn{10}{|c|}{ Expedition in Bangka Archipelago and Adjacent Islands } \\
\hline Higher Taxon Affiliation & Identifier & Species Name & $\begin{array}{c}\text { Exp } \\
\text { Bangka } \\
\text { 2017-2018 }\end{array}$ & $\begin{array}{c}\text { Exp Bunaken } \\
2015-2017\end{array}$ & $\begin{array}{c}\text { Exp } \\
\text { Bunaken } \\
2003\end{array}$ & $\begin{array}{c}\text { Exp } \\
\text { Sangihe } \\
2016\end{array}$ & $\begin{array}{c}\text { Exp } \\
\text { Lembeh } \\
2018\end{array}$ & $\begin{array}{l}\text { Lembeh } \\
\text { Sea Slug } \\
\text { Forum } \\
2001-2010\end{array}$ & $\begin{array}{l}\text { Tonozuka } \\
2003\end{array}$ \\
\hline \multirow{16}{*}{$\begin{array}{l}\text { Discodorididae Bergh, } \\
1891\end{array}$} & & $\begin{array}{c}\text { Asteronotus cespitosus (van Hasselt, } \\
1824) *\end{array}$ & $x$ & $x$ & & & & & $x$ \\
\hline & & $\begin{array}{l}\text { Asteronotus mimeticus } \\
\text { Gosliner and Valdés, } 2002\end{array}$ & $x$ & $x$ & & & & & \\
\hline & & Atagema intecta (Kelaart, 1859) & $x$ & & & & & & $x$ \\
\hline & Disp1_Bu-1 & $\begin{array}{l}\text { Diaulula sp. (Diaulula sp. } 1 \text { in } \\
\text { Gosliner et al. [24]: 197) }\end{array}$ & & $x$ & & & & & \\
\hline & & $\begin{array}{c}\text { Carminodoris estrelyado } \\
\text { (Gosliner and Behrens, 1998) }\end{array}$ & & & & & & $\mathrm{x}$ & $x$ \\
\hline & & Discodoris cebuensis Bergh, 1877 & $\mathrm{x}$ & & & & & & \\
\hline & & $\begin{array}{c}\text { Halgerda batangas Carlson and } \\
\text { Hoff, } 2000\end{array}$ & $x$ & $x$ & $x$ & & $x$ & $x$ & \\
\hline & & Halgerda carlsoni Rudman, 1978 & $x$ & $x$ & & & & & \\
\hline & & $\begin{array}{l}\text { Halgerda okinawa Carlson and Hoff, } \\
2000\end{array}$ & & & & & & $x$ & \\
\hline & & Halgerda tessellate (Bergh, 1880) & & $x$ & & & & & \\
\hline & & Jorunna funebris Kelaart, 1859 & $x$ & & & & $x$ & $x$ & \\
\hline & & Paradoris liturata (Bergh, 1905) & $\mathrm{x}$ & & & & & $\mathrm{x}$ & \\
\hline & & $\begin{array}{c}\text { Platydoris ellioti } \\
\text { (Alder and Hancock, 1864) }\end{array}$ & & & & & & $x$ & \\
\hline & & $\begin{array}{c}\text { Platydoris formosa } \\
\text { (Alder and Hancock, 1864) }\end{array}$ & & & & & & & $x$ \\
\hline & & $\begin{array}{c}\text { Platydoris inframaculata (Abraham, } \\
\text { 1877) }\end{array}$ & & & & & & $x$ & \\
\hline & & Platydoris sanguinea Bergh, 1905 & $x$ & $x$ & & & & & \\
\hline
\end{tabular}


Table 3. Cont

\begin{tabular}{|c|c|c|c|c|c|c|c|c|c|}
\hline \multicolumn{10}{|c|}{ Expedition in Bangka Archipelago and Adjacent Islands } \\
\hline \multirow[t]{17}{*}{ Higher Taxon Affiliation } & Identifier & Species Name & $\begin{array}{c}\text { Exp } \\
\text { Bangka } \\
\text { 2017-2018 }\end{array}$ & $\begin{array}{c}\text { Exp Bunaken } \\
2015-2017\end{array}$ & $\begin{array}{c}\text { Exp } \\
\text { Bunaken } \\
2003\end{array}$ & $\begin{array}{c}\text { Exp } \\
\text { Sangihe } \\
2016\end{array}$ & $\begin{array}{c}\text { Exp } \\
\text { Lembeh } \\
2018\end{array}$ & $\begin{array}{l}\text { Lembeh } \\
\text { Sea Slug } \\
\text { Forum } \\
2001-2010\end{array}$ & $\begin{array}{l}\text { Tonozuka } \\
2003\end{array}$ \\
\hline & & Sclerodoris tuberculate Eliot, 1904 & & & & & & $x$ & \\
\hline & Scsp2_16Bu-1 & $\begin{array}{l}\text { Sclerodoris sp. (Sclerodoris sp. } 2 \text { in } \\
\text { Gosliner et al. [24]: 195) }\end{array}$ & & $x$ & & & & & \\
\hline & & $\begin{array}{c}\text { Taringa halgerda } \\
\text { Gosliner and Behrens, } 1998\end{array}$ & & $\mathrm{x}$ & & & & & \\
\hline & Dosp17Bu-1 & Discodorididae sp. & & $\mathrm{x}$ & & & & & \\
\hline & Dosp17Bu-2 & Discodorididae sp. & & $\mathrm{x}$ & & & & & \\
\hline & & Ardeadoris averni (Rudman, 1985) & & & & & & & $x$ \\
\hline & & Ardeadoris cruenta (Rudman, 1986) & & & & & & $\mathrm{X}$ & \\
\hline & & Ceratosoma bicolor Baba, 1949 & & & & & & $x$ & \\
\hline & & Ceratosoma tenue Abraham, $1876^{*}$ & $x$ & & & & & $x$ & $x$ \\
\hline & & $\begin{array}{l}\text { Ceratosoma trilobatum (J.E. Gray, } \\
\text { 1827) }\end{array}$ & & & & & & $x$ & $x$ \\
\hline & $\begin{array}{l}\text { Cesp2_15Bu-3 } \\
\text { Cesp1_17Bu-1 }\end{array}$ & $\begin{array}{l}\text { Ceratosoma sp. (Ceratosoma sp. } 1 \text { in } \\
\text { Gosliner et al. [24]: 266) }\end{array}$ & & $x$ & & & & & \\
\hline & & Chromodoris annae Bergh, 1877 & $x$ & $x$ & $x$ & $x$ & $x$ & $x$ & \\
\hline & & $\begin{array}{l}\text { Chromodoris cf. boucheti Rudman, } \\
1982\end{array}$ & & $x$ & & & & & \\
\hline & & $\begin{array}{l}\text { Chromodoris colemani Rudman, } \\
1982\end{array}$ & & & & & & $\mathrm{x}$ & \\
\hline & & $\begin{array}{c}\text { Chromodoris dianae } \\
\text { Gosliner and Behrens, } 1998\end{array}$ & $x$ & $x$ & $x$ & $x$ & & & \\
\hline & & $\begin{array}{l}\text { Chromodoris elisabethina Bergh, } \\
1877^{*}\end{array}$ & $x$ & & & & & & \\
\hline
\end{tabular}


Table 3. Cont

\begin{tabular}{|c|c|c|c|c|c|c|c|c|c|}
\hline \multicolumn{10}{|c|}{ Expedition in Bangka Archipelago and Adjacent Islands } \\
\hline Higher Taxon Affiliation & Identifier & Species Name & $\begin{array}{c}\text { Exp } \\
\text { Bangka } \\
\text { 2017-2018 }\end{array}$ & $\begin{array}{c}\text { Exp Bunaken } \\
2015-2017\end{array}$ & $\begin{array}{c}\text { Exp } \\
\text { Bunaken } \\
2003\end{array}$ & $\begin{array}{c}\text { Exp } \\
\text { Sangihe } \\
2016\end{array}$ & $\begin{array}{c}\text { Exp } \\
\text { Lembeh } \\
2018\end{array}$ & $\begin{array}{l}\text { Lembeh } \\
\text { Sea Slug } \\
\text { Forum } \\
2001-2010\end{array}$ & $\begin{array}{l}\text { Tonozuka } \\
2003\end{array}$ \\
\hline \multirow{16}{*}{$\begin{array}{c}\text { Chromodorididae Bergh, } \\
1891\end{array}$} & & Chromodoris lochi Rudman, 1982 & $\mathrm{x}$ & $\mathrm{x}$ & $\mathrm{x}$ & & $x$ & $x$ & $x$ \\
\hline & & $\begin{array}{c}\text { Chromodoris magnifica } \\
\text { (Quoy and Gaimard, 1832) }\end{array}$ & $\mathrm{x}$ & & & & & $\mathrm{x}$ & \\
\hline & & $\begin{array}{l}\text { Chromodoris cf. michaeli } \\
\text { Gosliner and Behrens, } 1998\end{array}$ & & $\mathrm{x}$ & & & & & \\
\hline & & $\begin{array}{c}\text { Chromodoris quadricolor } \\
\text { (Rüppell and Leuckart, 1830) }\end{array}$ & $x$ & & & & & & \\
\hline & & Chromodoris strigata Rudman,1982 & $x$ & $\mathrm{x}$ & $\mathrm{x}$ & $\mathrm{x}$ & & $\mathrm{x}$ & \\
\hline & & Chromodoris willani Rudman, 1982 & $\mathrm{x}$ & $\mathrm{x}$ & $\mathrm{x}$ & & & $\mathrm{X}$ & \\
\hline & & $\begin{array}{c}\text { Doriprismatica atromarginata } \\
\text { (Cuvier, 1804) }\end{array}$ & $x$ & & & & & & \\
\hline & & Doriprismatica sibogae Berg, 1905 & $\mathrm{x}$ & & & & & & \\
\hline & & $\begin{array}{l}\text { Doriprismatica stellata (Rudman, } \\
\text { 1986) }\end{array}$ & & $\mathrm{x}$ & & & & & \\
\hline & & Glossodoris cincta (Bergh, 1888) & $x$ & $x$ & & $x$ & $x$ & $x$ & \\
\hline & & $\begin{array}{c}\text { Glossodoris hikuerensis (Pruvot-Fol, } \\
1954)\end{array}$ & & $x$ & & & & & \\
\hline & & $\begin{array}{c}\text { Glossodoris rufomarginata (Bergh, } \\
1890)^{*}\end{array}$ & $x$ & & & & & $x$ & \\
\hline & & $\begin{array}{l}\text { Goniobranchus aureopurpureus } \\
\text { (Collingwood, 1881) }\end{array}$ & & & & & & $X$ & \\
\hline & & Goniobranchus coi (Risbec, 1956) & $\mathrm{x}$ & & & & & & \\
\hline & & Goniobranchus decorus (Pease, 1860) & & & $\mathrm{x}$ & & & & \\
\hline & & Goniobranchus fidelis (Kelaart, 1858) & $\mathrm{x}$ & $\mathrm{x}$ & & & $\mathrm{x}$ & $\mathrm{x}$ & \\
\hline
\end{tabular}


Table 3. Cont

\begin{tabular}{|c|c|c|c|c|c|c|c|c|c|}
\hline \multicolumn{10}{|c|}{ Expedition in Bangka Archipelago and Adjacent Islands } \\
\hline Higher Taxon Affiliation & Identifier & Species Name & $\begin{array}{c}\text { Exp } \\
\text { Bangka } \\
\text { 2017-2018 }\end{array}$ & $\begin{array}{l}\text { Exp Bunaken } \\
\text { 2015-2017 }\end{array}$ & $\begin{array}{c}\text { Exp } \\
\text { Bunaken } \\
2003\end{array}$ & $\begin{array}{c}\text { Exp } \\
\text { Sangihe } \\
2016\end{array}$ & $\begin{array}{c}\text { Exp } \\
\text { Lembeh } \\
2018\end{array}$ & $\begin{array}{c}\text { Lembeh } \\
\text { Sea Slug } \\
\text { Forum } \\
2001-2010\end{array}$ & $\begin{array}{c}\text { Tonozuka } \\
2003\end{array}$ \\
\hline & & $\begin{array}{c}\text { Goniobranchus geometricus (Risbec, } \\
\text { 1928) }\end{array}$ & $\mathrm{x}$ & $\mathrm{X}$ & $\mathrm{x}$ & $\mathrm{x}$ & $\mathrm{X}$ & $\mathrm{x}$ & \\
\hline & & $\begin{array}{l}\text { Goniobranchus hintuanensis } \\
\text { (Gosliner and Behrens, 1998) }\end{array}$ & & & & & & $\mathrm{x}$ & \\
\hline & & $\begin{array}{c}\text { Goniobranchus kuniei (Pruvot-Fol, } \\
\text { 1930) }\end{array}$ & $\mathrm{x}$ & & & & & $\mathrm{x}$ & \\
\hline & & $\begin{array}{c}\text { Goniobranchus preciosus (Kelaart, } \\
1858 \text { ) } \\
\end{array}$ & & & & & & $\mathrm{x}$ & \\
\hline & & $\begin{array}{l}\text { Goniobranchus reticulatus } \\
\text { (Quoy and Gaimard, 1832) }\end{array}$ & $\mathrm{x}$ & $\mathrm{X}$ & & $\mathrm{x}$ & $\mathrm{X}$ & $\mathrm{x}$ & \\
\hline & & $\begin{array}{c}\text { Goniobranchus roboi } \\
\text { (Gosliner and Behrens, 1998) }\end{array}$ & & & & & & $\mathrm{x}$ & \\
\hline & & $\begin{array}{c}\text { Goniobranchus tinctorius } \\
\text { (Rüppell and Leuckart, 1830) }\end{array}$ & & & $\mathrm{x}$ & & & & \\
\hline & & $\begin{array}{c}\text { Goniobranchus verrieri (Crosse, } \\
1875 \text { ) }\end{array}$ & $\mathrm{x}$ & & & & & $\mathrm{x}$ & \\
\hline & Gosp40_16Bu-1 & $\begin{array}{l}\text { Goniobranchus sp. (Goniobranchus } \\
\text { sp. } 40 \text { in Gosliner et al. [24]: 230) }\end{array}$ & & $\mathrm{x}$ & & & & & \\
\hline & & $\begin{array}{l}\text { Hypselodoris apolegma (Yonow, } \\
\text { 2001) }\end{array}$ & $\mathrm{X}$ & $\mathrm{X}$ & $\mathrm{x}$ & & & $\mathrm{x}$ & \\
\hline & & $\begin{array}{l}\text { Hypselodoris bullockii } \\
\text { (Collingwood, 1881)* }\end{array}$ & $\mathrm{x}$ & & & & & & \\
\hline & Hysp1_17Ba-1 & $\begin{array}{c}\text { Hypselodoris cerisae } \\
\text { Gosliner and Johnson, } 2018\end{array}$ & $\mathrm{x}$ & & & & & $\mathrm{x}$ & \\
\hline & & $\begin{array}{l}\text { Hypselodoris decorata } \\
\text { Gosliner and Johnson, } 2018\end{array}$ & $\mathrm{x}$ & & & & & & \\
\hline
\end{tabular}


Table 3. Cont

\begin{tabular}{|c|c|c|c|c|c|c|c|c|c|}
\hline \multicolumn{10}{|c|}{ Expedition in Bangka Archipelago and Adjacent Islands } \\
\hline Higher Taxon Affiliation & Identifier & Species Name & $\begin{array}{c}\text { Exp } \\
\text { Bangka } \\
\text { 2017-2018 }\end{array}$ & $\begin{array}{l}\text { Exp Bunaken } \\
\text { 2015-2017 }\end{array}$ & $\begin{array}{c}\text { Exp } \\
\text { Bunaken } \\
2003\end{array}$ & $\begin{array}{c}\text { Exp } \\
\text { Sangihe } \\
2016\end{array}$ & $\begin{array}{c}\text { Exp } \\
\text { Lembeh } \\
2018\end{array}$ & $\begin{array}{l}\text { Lembeh } \\
\text { Sea Slug } \\
\text { Forum } \\
2001-2010\end{array}$ & $\begin{array}{c}\text { Tonozuka } \\
2003\end{array}$ \\
\hline & & Hypselodoris emma Rudman, 1977 * & $x$ & & & & & & \\
\hline & Chromos18Ba-1 & $\begin{array}{c}\text { Hypselodoris iacula } \\
\text { Gosliner and Johnson, } 1999\end{array}$ & $\mathrm{x}$ & & & & & & \\
\hline & & $\begin{array}{c}\text { Hypselodoris iba } \\
\text { Gosliner and Johnson, } 2018\end{array}$ & & & & & & & $\mathrm{x}$ \\
\hline & & $\begin{array}{l}\text { Hypselodoris infucata } \\
\text { (Rüppell and Leuckart, 1830) }\end{array}$ & & & & & & & $\mathrm{x}$ \\
\hline & & Hypselodoris kanga Rudman, 1977 & & & & & & $x$ & \\
\hline & & $\begin{array}{l}\text { Hypselodoris cf. krakatoa } \\
\text { Gosliner and Johnson, } 1999\end{array}$ & & & & & & $\mathrm{X}$ & \\
\hline & Hysp19_17Ba-1-2 & $\begin{array}{c}\text { Hypselodoris lacuna } \\
\text { Gosliner and Johnson, } 2018\end{array}$ & $\mathrm{x}$ & & & & & & \\
\hline & & Hypselodoris maculosa (Pease, 1871) & $x$ & $x$ & & & & $x$ & $x$ \\
\hline & & Hypselodoris zephyra (Eliot, 1904) & $x$ & & & & & & \\
\hline & & $\begin{array}{c}\text { Hypselodoris nigrostriata (Eliot, } \\
1904 \text { ) }\end{array}$ & $\mathrm{X}$ & & & & & $\mathrm{x}$ & \\
\hline & & $\begin{array}{c}\text { Hypselodoris purpureomaculosa } \\
\text { Hamatani, } 1995\end{array}$ & & & & & & $\mathrm{x}$ & $\mathrm{x}$ \\
\hline & & Hypselodoris tryoni (Garret, 1873) & $x$ & $x$ & $x$ & $x$ & $x$ & $x$ & \\
\hline & Thsp1-18Ba-1 & Hypselodoris sp. a & $x$ & $x$ & & & & $x$ & \\
\hline & Hysp16Bu-1 & Hypselodoris sp. & & $X$ & & & & & \\
\hline & Hysp2_16Bu-1 & Hypselodoris sp. & & $x$ & & & & & \\
\hline
\end{tabular}


Table 3. Cont

\begin{tabular}{|c|c|c|c|c|c|c|c|c|c|}
\hline \multicolumn{10}{|c|}{ Expedition in Bangka Archipelago and Adjacent Islands } \\
\hline \multirow[t]{12}{*}{ Higher Taxon Affiliation } & Identifier & Species Name & $\begin{array}{c}\text { Exp } \\
\text { Bangka } \\
\text { 2017-2018 }\end{array}$ & $\begin{array}{l}\text { Exp Bunaken } \\
\text { 2015-2017 }\end{array}$ & $\begin{array}{c}\text { Exp } \\
\text { Bunaken } \\
2003\end{array}$ & $\begin{array}{c}\text { Exp } \\
\text { Sangihe } \\
2016\end{array}$ & $\begin{array}{c}\text { Exp } \\
\text { Lembeh } \\
2018\end{array}$ & $\begin{array}{l}\text { Lembeh } \\
\text { Sea Slug } \\
\text { Forum } \\
2001-2010\end{array}$ & $\begin{array}{c}\text { Tonozuka } \\
2003\end{array}$ \\
\hline & & $\begin{array}{c}\text { Mexichromis aurora } \\
\text { (Johnson and Gosliner, 1998) }\end{array}$ & $x$ & & & & & & \\
\hline & & $\begin{array}{c}\text { Mexichromis multituberculata (Baba, } \\
\text { 1953) }\end{array}$ & & & & & & $x$ & \\
\hline & & $\begin{array}{l}\text { Mexichromis trilineata v(A. Adams } \\
\text { and Reeve, 1850) }\end{array}$ & $x$ & & & & & $\mathrm{x}$ & \\
\hline & & Miamira sinuata (van Hasselt, 1824) & & $x$ & $x$ & & & & \\
\hline & Dorid18Ba-1 & Miamira magnifica & $x$ & & & & & & \\
\hline & Misp17Ba_1 & Miamira sp. a & $x$ & & & & & & \\
\hline & & Verconia simplex Pease, 1871 & $x$ & & & & & & \\
\hline & & Thorunna florens (Baba, 1949) & & & & & & $x$ & \\
\hline & & Thorunna furtiva Bergh, 1878 & $x$ & $x$ & & & & & \\
\hline & & Verconia varians (Pease, 1871) & & $x$ & $x$ & & & & \\
\hline & Dosp17Bu-4 & Verconia sp. & & $x$ & & & & & \\
\hline \multirow{6}{*}{$\begin{array}{c}\text { Dendrodorididae } \\
\text { O'Donoghue, } 1924 \text { (1864) }\end{array}$} & & Dendrodoris albobrunnea Allan, 1933 & & $x$ & & & & & \\
\hline & & $\begin{array}{c}\text { Dendrodoris carbunculosa (Kelaart, } \\
1858 \text { ) }\end{array}$ & & & & & & $\mathrm{x}$ & \\
\hline & & Dendrodoris elongata Baba, 1936 & & & $x$ & & & & \\
\hline & & Dendrodoris guttata (Odhner, 1917) & & & & & & $x$ & \\
\hline & & Dendrodoris nigra (Stimpson, 1855) & $x$ & $x$ & & & & & \\
\hline & Rosp17Bu-1 & Dendrodoris sp. & & $\mathrm{x}$ & & & & & \\
\hline
\end{tabular}


Table 3. Cont

\begin{tabular}{|c|c|c|c|c|c|c|c|c|c|}
\hline \multicolumn{10}{|c|}{ Expedition in Bangka Archipelago and Adjacent Islands } \\
\hline Higher Taxon Affiliation & Identifier & Species Name & $\begin{array}{c}\text { Exp } \\
\text { Bangka } \\
\text { 2017-2018 }\end{array}$ & $\begin{array}{l}\text { Exp Bunaken } \\
\text { 2015-2017 }\end{array}$ & $\begin{array}{c}\text { Exp } \\
\text { Bunaken } \\
2003\end{array}$ & $\begin{array}{c}\text { Exp } \\
\text { Sangihe } \\
2016\end{array}$ & $\begin{array}{c}\text { Exp } \\
\text { Lembeh } \\
2018\end{array}$ & $\begin{array}{l}\text { Lembeh } \\
\text { Sea Slug } \\
\text { Forum } \\
\text { 2001-2010 }\end{array}$ & $\begin{array}{c}\text { Tonozuka } \\
2003\end{array}$ \\
\hline \multirow{17}{*}{$\begin{array}{l}\text { Phyllidiidae Rafinesque, } \\
1814\end{array}$} & & $\begin{array}{l}\text { Phyllidia cf. babai Brunckhorst, } \\
1993^{*}\end{array}$ & $\mathrm{x}$ & & & & & $\mathrm{x}$ & $\mathrm{x}$ \\
\hline & & Phyllidia coelestis Bergh, 1905 & $x$ & $x$ & $x$ & $x$ & & & \\
\hline & & Phyllidia elegans Bergh, 1869 & $x$ & $x$ & $x$ & & $x$ & $\mathrm{x}$ & \\
\hline & & $\begin{array}{c}\text { Phyllidia exquisita Brunckhorst, } \\
1993\end{array}$ & $\mathrm{x}$ & & & & & $\mathrm{x}$ & \\
\hline & & $\begin{array}{c}\text { Phyllidia madangensis Brunckhorst, } \\
1993\end{array}$ & & & & $\mathrm{x}$ & & & \\
\hline & & Phyllidia ocellata Cuvier, 1804 & $\mathrm{x}$ & $\mathrm{x}$ & $x$ & $x$ & & $x$ & \\
\hline & & Phyllidia picta Pruvot-Fol, 1957 & $x$ & & & $x$ & & & \\
\hline & & $\begin{array}{c}\text { Phyllidia polkadotsa Brunckhorst, } \\
1993\end{array}$ & & & & & & $\mathrm{x}$ & \\
\hline & Phsp18Ba-2-3 & Phyllidia sp. a & $\mathrm{x}$ & $\mathrm{x}$ & & $\mathrm{x}$ & & & \\
\hline & & Phyllidia varicosa Lamarck, 1801 & $x$ & $x$ & $x$ & $x$ & $x$ & $x$ & \\
\hline & & Phyllidiella annulata (Gray, 1853) & $x$ & $x$ & & & & & \\
\hline & & Phyllidiella lizae Brunckhorst, 1993 & $x$ & & $x$ & $x$ & & & \\
\hline & & Phyllidiella nigra (van Hasselt 1824) & $x$ & & $x$ & $x$ & & & \\
\hline & & Phyllidiella pustulosa (Cuvier, 1804) & $x$ & $x$ & $x$ & $x$ & $x$ & $x$ & \\
\hline & & $\begin{array}{l}\text { Phyllidiopsis annae Brunckhorst, } \\
1993\end{array}$ & $x$ & & & & & $\mathrm{x}$ & \\
\hline & Phssp.aBa-1 & $\begin{array}{c}\text { Phyllidiopsis cf. burni Brunckhorst, } \\
1983\end{array}$ & $x$ & & & & & & \\
\hline & & $\begin{array}{c}\text { Phyllidiopsis krempfi Pruvot-Fol, } \\
1957\end{array}$ & $\mathrm{x}$ & & & $\mathrm{x}$ & & & \\
\hline
\end{tabular}


Table 3. Cont

\begin{tabular}{|c|c|c|c|c|c|c|c|c|c|}
\hline \multicolumn{10}{|c|}{ Expedition in Bangka Archipelago and Adjacent Islands } \\
\hline \multirow[t]{5}{*}{ Higher Taxon Affiliation } & Identifier & Species Name & $\begin{array}{c}\text { Exp } \\
\text { Bangka } \\
\text { 2017-2018 }\end{array}$ & $\begin{array}{l}\text { Exp Bunaken } \\
2015-2017\end{array}$ & $\begin{array}{l}\text { Exp } \\
\text { Bunaken } \\
2003\end{array}$ & $\begin{array}{c}\text { Exp } \\
\text { Sangihe } \\
2016\end{array}$ & $\begin{array}{c}\text { Exp } \\
\text { Lembeh } \\
2018\end{array}$ & $\begin{array}{l}\text { Lembeh } \\
\text { Sea Slug } \\
\text { Forum } \\
2001-2010\end{array}$ & $\begin{array}{c}\text { Tonozuka } \\
2003\end{array}$ \\
\hline & & $\begin{array}{c}\text { Phyllidiopsis pipeki Brunckhorst, } \\
1993\end{array}$ & & $x$ & & & & & \\
\hline & & $\begin{array}{c}\text { Phyllidiopsis shireenae Brunckhorst, } \\
1990\end{array}$ & & & & $\mathrm{X}$ & & $\mathrm{x}$ & \\
\hline & & $\begin{array}{c}\text { Phyllidiopsis sphingis Brunckhorst, } \\
1993\end{array}$ & & $x$ & & & & & \\
\hline & & Phyllidiopsis xishaensis (Lin, 1983) & $\mathrm{x}$ & $\mathrm{x}$ & $x$ & & & & \\
\hline \multicolumn{10}{|c|}{ Nudibranchia, Cladobranchia } \\
\hline \multirow{11}{*}{$\begin{array}{l}\text { Arminidae } \\
\text { Iredale and O'Donoghue, } \\
1923\end{array}$} & & $\begin{array}{l}\text { Dermatobranchus caeruleomaculatus } \\
\text { Gosliner and Fahey, } 2011^{*}\end{array}$ & $\mathrm{x}$ & & & & & & \\
\hline & & $\begin{array}{l}\text { Dermatobranchus diagonalis } \\
\text { Gosliner and Fahey, } 2011\end{array}$ & & $\mathrm{x}$ & & & & & \\
\hline & & $\begin{array}{l}\text { Dermatobranchus fasciatus } \\
\text { Gosliner and Fahey, } 2011\end{array}$ & & $x$ & & & & & \\
\hline & & $\begin{array}{l}\text { Dermatobranchus cf. kokonas } \\
\text { Gosliner and Fahey, } 2011\end{array}$ & & $\mathrm{x}$ & & & & & \\
\hline & & $\begin{array}{l}\text { Dermatobranchus cf. piperoides } \\
\text { Gosliner and Fahey, } 2011\end{array}$ & & $\mathrm{x}$ & & & & & \\
\hline & & $\begin{array}{l}\text { Dermatobranchus rodmani } \\
\text { Gosliner and Fahey, } 2011\end{array}$ & $x$ & & & & & & \\
\hline & & $\begin{array}{l}\text { Dermatobranchus pustulosus } \\
\text { van Hasselt, } 1824\end{array}$ & $\mathrm{x}$ & & & & & & \\
\hline & Desp8_17Bu-1 & $\begin{array}{c}\text { Dermatobranchus sp. } \\
\text { (Dermatobranchus sp. } 8 \text { in Gosliner } \\
\text { et al. [24]: } 302 \text { ) }\end{array}$ & & $\mathrm{x}$ & & & & & \\
\hline & Desp17Ba-1 & Dermatobranchus sp. & $\mathrm{x}$ & & & & & & \\
\hline & Desp.a18Ba-1-2 & Dermatobranchus sp. a & $x$ & & & & & & \\
\hline & Desp1_16Bu-1Des] & 8Ba-1 Dermatobranchus sp. & $x$ & $x$ & & & & & \\
\hline
\end{tabular}


Table 3. Cont

\begin{tabular}{|c|c|c|c|c|c|c|c|c|c|}
\hline \multicolumn{10}{|c|}{ Expedition in Bangka Archipelago and Adjacent Islands } \\
\hline Higher Taxon Affiliation & Identifier & Species Name & $\begin{array}{c}\text { Exp } \\
\text { Bangka } \\
\text { 2017-2018 }\end{array}$ & $\begin{array}{l}\text { Exp Bunaken } \\
\text { 2015-2017 }\end{array}$ & $\begin{array}{c}\text { Exp } \\
\text { Bunaken } \\
2003\end{array}$ & $\begin{array}{c}\text { Exp } \\
\text { Sangihe } \\
2016\end{array}$ & $\begin{array}{c}\text { Exp } \\
\text { Lembeh } \\
2018\end{array}$ & $\begin{array}{l}\text { Lembeh } \\
\text { Sea Slug } \\
\text { Forum } \\
2001-2010\end{array}$ & $\begin{array}{c}\text { Tonozuka } \\
2003\end{array}$ \\
\hline \multirow{3}{*}{ Proctonotidae Gray, 1853} & & $\begin{array}{l}\text { Janolus cf. mirabilis Baba and Abe, } \\
1970\end{array}$ & & $x$ & $x$ & & & & \\
\hline & & $\begin{array}{c}\text { Janolus savinkini } \\
\text { Martynov and Korshunova, } 2012\end{array}$ & & & & & & $\begin{array}{c}\mathrm{X} \\
\text { as Janolus sp. } \\
4\end{array}$ & \\
\hline & & $\begin{array}{l}\text { Janolus sp. } 1 \text { (in Gosliner et al. [24]: } \\
\qquad 305)^{*}\end{array}$ & $x$ & $x$ & & & & & \\
\hline $\begin{array}{l}\text { Scyllaeidae Alder and } \\
\text { Hancock, } 1855\end{array}$ & Cross16Bu-1-8 & $\begin{array}{c}\text { Crosslandia daedali } \\
\text { Poorman and Mulliner, } 1981\end{array}$ & & $x$ & & & & & \\
\hline \multirow{3}{*}{ Bornellidae Bergh, 1874} & & Bornella anguilla Johnson, 1984 & $x$ & & & & & & \\
\hline & & $\begin{array}{l}\text { Bornella stellifera (A. Adams and } \\
\text { Reeve [in A. Adams], 1848) }\end{array}$ & $x$ & & $x$ & & & & \\
\hline & & $\begin{array}{l}\text { Pseudovermis cf. mortoni Challis, } \\
1969\end{array}$ & & & $x$ & & & & \\
\hline $\begin{array}{l}\text { Pseudovermidae Thiele, } \\
1931\end{array}$ & Mesp.a18Ba-1 & Melibe bucephala Bergh, 1902 & $x$ & & & & & & \\
\hline \multirow{3}{*}{$\begin{array}{l}\text { Tethydidae Rafinesque, } \\
1815\end{array}$} & & Melibe engeli Risbec, 1937 & $x$ & & & & & & \\
\hline & & Melibe viridis (Kelaart, 1858) & & & & & & $x$ & \\
\hline & & Doto ussi Ortea, 1982 & $x$ & $x$ & & & & & \\
\hline \multirow{5}{*}{ Dotidae Gray, 1853} & & $\begin{array}{c}\text { Kabeiro rubroreticulata } \\
\text { Shipman and Gosliner, } 2015\end{array}$ & & $\mathrm{x}$ & & & & & \\
\hline & Dotosp15Bu-1 & Kabeiro sp. & & $x$ & & & & & \\
\hline & $\begin{array}{l}\text { Kasp16Bu-1-15+17 } \\
\text { Kasp17Bu-1 }\end{array}$ & Kabeiro sp. & & $x$ & & & & & \\
\hline & Kasp16Bu-16 & Kabeiro sp. & & $x$ & & & & & \\
\hline & & Marianina rosea (Pruvot-Fol, 1930) & & $\mathrm{X}$ & & & & & \\
\hline
\end{tabular}


Table 3. Cont

\begin{tabular}{|c|c|c|c|c|c|c|c|c|c|}
\hline \multicolumn{10}{|c|}{ Expedition in Bangka Archipelago and Adjacent Islands } \\
\hline Higher Taxon Affiliation & Identifier & Species Name & $\begin{array}{c}\text { Exp } \\
\text { Bangka } \\
\text { 2017-2018 }\end{array}$ & $\begin{array}{l}\text { Exp Bunaken } \\
\text { 2015-2017 }\end{array}$ & $\begin{array}{c}\text { Exp } \\
\text { Bunaken } \\
2003\end{array}$ & $\begin{array}{l}\text { Exp } \\
\text { Sangihe } \\
2016\end{array}$ & $\begin{array}{l}\text { Exp } \\
\text { Lembeh } \\
2018\end{array}$ & $\begin{array}{l}\text { Lembeh } \\
\text { Sea Slug } \\
\text { Forum } \\
\text { 2001-2010 }\end{array}$ & $\begin{array}{c}\text { Tonozuka } \\
2003\end{array}$ \\
\hline \multirow{6}{*}{ Tritoniidae Lamarck, 1809} & Masp2.18Ba-1 & $\begin{array}{l}\text { Marionia sp. } 2 \text { (in Gosliner } \\
\text { et al. [24]: } 324 \text { ) }\end{array}$ & $x$ & & & & & & \\
\hline & Trsp3_17Ba-1 & $\begin{array}{l}\text { Tritonia sp. } 3 \text { (in Gosliner et al. [24]: } \\
\text { 320) }\end{array}$ & $x$ & & & & & & \\
\hline & Trsp8_16Bu-1 & $\begin{array}{c}\text { Tritonia sp. } \\
\text { (Tritonia sp. } 9 \text { in Gosliner } \\
\text { et al. [24]: } 321 \text { ) }\end{array}$ & & $x$ & & & & & \\
\hline & Trisp10_16Bu-1 & $\begin{array}{l}\text { Tritonia sp. } \\
\text { (Tritonia sp. } 10 \text { in Gosliner } \\
\text { et al. [24]: } 321 \text { ) }\end{array}$ & & $x$ & & & & & \\
\hline & Trsp16Bu-1 & Tritonia sp. & & $x$ & & & & & \\
\hline & & Embletonia gracilis Risbec, 1928 & & & $x$ & & & & \\
\hline Embletoniidae & & $\begin{array}{c}\text { Coryphellina delicate } \\
\text { (Gosliner and Willan, 1991) }\end{array}$ & & $x$ & & & & & \\
\hline \multirow{6}{*}{ Flabellinidae Bergh, 1889} & & $\begin{array}{c}\text { Coryphellina exoptata } \\
\text { (Gosliner and Willan, 1991) }\end{array}$ & $x$ & $\mathrm{X}$ & $\mathrm{x}$ & & & $\mathrm{x}$ & \\
\hline & & $\begin{array}{l}\text { Coryphellina rubrolineata } \\
\text { O'Donoghue, } 1929\end{array}$ & $\mathrm{x}$ & $\mathrm{x}$ & & & $x$ & $x$ & \\
\hline & Flsp2-18Ba-1 & $\begin{array}{c}\text { Flabellina sp. } 2 \\
\text { (in Gosliner et al. [24]: 333) }\end{array}$ & $x$ & & & & & & \\
\hline & Flsp3_17Ba-1 & $\begin{array}{c}\text { Flabellina sp. } 3 \\
\text { (in Gosliner et al. [24]: 333) }\end{array}$ & $x$ & & & & & & \\
\hline & & Flabellina sp. 8 (Sea Slug Forum) & & & & & & $x$ & \\
\hline & & Samla bicolor (Kelaart, 1858) & & $\mathrm{x}$ & & & & & \\
\hline
\end{tabular}


Table 3. Cont

\begin{tabular}{|c|c|c|c|c|c|c|c|c|c|}
\hline \multicolumn{10}{|c|}{ Expedition in Bangka Archipelago and Adjacent Islands } \\
\hline Higher Taxon Affiliation & Identifier & Species Name & $\begin{array}{c}\text { Exp } \\
\text { Bangka } \\
\text { 2017-2018 }\end{array}$ & $\begin{array}{c}\text { Exp Bunaken } \\
\text { 2015-2017 }\end{array}$ & $\begin{array}{c}\text { Exp } \\
\text { Bunaken } \\
2003\end{array}$ & $\begin{array}{c}\text { Exp } \\
\text { Sangihe } \\
2016\end{array}$ & $\begin{array}{c}\text { Exp } \\
\text { Lembeh } \\
2018\end{array}$ & $\begin{array}{l}\text { Lembeh } \\
\text { Sea Slug } \\
\text { Forum } \\
\text { 2001-2010 } \\
\end{array}$ & $\begin{array}{l}\text { Tonozuka } \\
2003\end{array}$ \\
\hline \multirow{3}{*}{$\begin{array}{l}\text { Samlidae Korshunova, } \\
\text { Martynov, Bakken, } \\
\text { Evertsen, Fletcher, } \\
\text { Mudianta, Saito, Lundin, } \\
\text { Schrödl and Picton, } 2017\end{array}$} & & Samla bicolor (Kelaart, 1858) & & & & & & $x$ & \\
\hline & & $\begin{array}{c}\text { Samla riwo } \\
\text { (Gosliner and Willan, 1991) }\end{array}$ & $\mathrm{x}$ & $\mathrm{x}$ & & & & $\mathrm{x}$ & \\
\hline & & Phestilla lugubris (Bergh, 1870) & & $x$ & & & & & \\
\hline \multirow[t]{3}{*}{$\begin{array}{r}\text { Trinchesiidae F. } \\
\text { Nordsieck, } 1972 \\
\end{array}$} & & Phestilla minor Rudman, 1981 & & $\mathrm{x}$ & & & & & \\
\hline & & $\begin{array}{c}\text { Trinchesia yamasui (Hamatani, } \\
\text { 1993) }\end{array}$ & $\mathrm{x}$ & & & & & & \\
\hline & & $\begin{array}{l}\text { Eubranchus sp. (Eubranchus sp. } 22 \\
\text { in Gosliner et al. [24]: 341) }\end{array}$ & $\mathrm{x}$ & $\mathrm{x}$ & & & & & \\
\hline $\begin{array}{l}\text { Eubranchidae Odhner, } \\
1934\end{array}$ & Cusp4_16Bu-1 & $\begin{array}{l}\text { Cuthona sp. (Cuthona sp. } 4 \text { in } \\
\text { Gosliner et al. [24]: 343) }\end{array}$ & & $\mathrm{x}$ & & & & & \\
\hline \multirow{4}{*}{ Cuthonidae Odhner, 1934} & Cusp54_16Bu-1 & $\begin{array}{l}\text { Cuthona sp. (Cuthona sp. } 54 \text { in } \\
\text { Gosliner et al. [24]: 353) }\end{array}$ & & $\mathrm{x}$ & & & & & \\
\hline & & $\begin{array}{l}\text { Cuthona sp. } 57 \text { (in Gosliner } \\
\text { et al. [24]: 353) }\end{array}$ & $x$ & & & & & & \\
\hline & Cusp65_16Bu-1 & $\begin{array}{l}\text { Cuthona sp. (Cuthona cf. sp. } 65 \text { in } \\
\text { Gosliner et al. [24]: 343) }\end{array}$ & & $\mathrm{x}$ & & & & & \\
\hline & Aeol18Ba-1 & Aeolidia sp. & $x$ & & $x$ & $x$ & & & \\
\hline \multirow{4}{*}{ Aeolidiidae Gray, 1827} & & Bulbaeolidia alba (Risbec, 1928) & & $\mathrm{x}$ & & & & $x$ & \\
\hline & & Baeolidia australis (Rudman, 1982) & & & & & & $\mathrm{X}$ & \\
\hline & & Cerberilla ambonensis Bergh, 1905 & & & & & & & $\mathrm{x}$ \\
\hline & Ansp17Ba-1 & Antonietta sp. & $x$ & & & & & & \\
\hline
\end{tabular}


Table 3. Cont

\begin{tabular}{|c|c|c|c|c|c|c|c|c|c|}
\hline \multicolumn{10}{|c|}{ Expedition in Bangka Archipelago and Adjacent Islands } \\
\hline Higher Taxon Affiliation & Identifier & Species Name & $\begin{array}{c}\text { Exp } \\
\text { Bangka } \\
\text { 2017-2018 }\end{array}$ & $\begin{array}{l}\text { Exp Bunaken } \\
\text { 2015-2017 }\end{array}$ & $\begin{array}{c}\text { Exp } \\
\text { Bunaken } \\
2003\end{array}$ & $\begin{array}{c}\text { Exp } \\
\text { Sangihe } \\
2016\end{array}$ & $\begin{array}{c}\text { Exp } \\
\text { Lembeh } \\
2018\end{array}$ & $\begin{array}{l}\text { Lembeh } \\
\text { Sea Slug } \\
\text { Forum } \\
2001-2010\end{array}$ & $\begin{array}{c}\text { Tonozuka } \\
2003\end{array}$ \\
\hline \multirow{15}{*}{ Facelinidae Bergh, 1889} & & Caloria indica (Bergh, 1896) & $x$ & $x$ & & & & & \\
\hline & & $\begin{array}{c}\text { Caloria sp. (Caloria sp. } 1 \\
\text { in Gosliner et al. [24]: } 362 \text { ) }\end{array}$ & & $x$ & & & & & \\
\hline & & $\begin{array}{l}\text { Cratena sp. (Cratena sp. } 5 \text { in } \\
\text { Gosliner et al. [24]: } 383 \text { ) }\end{array}$ & & $x$ & & & & & \\
\hline & Crsp17Ba-1 & Cratena sp. & $\mathrm{x}$ & & & & & & \\
\hline & & Facelina rhodopos Yonow, 2000 & & $\mathrm{x}$ & & & & & \\
\hline & Fasp3_16Bu-1+3-5 & $\begin{array}{l}\text { Facelina sp. (Facelina sp. } 3 \text { in } \\
\text { Gosliner et al. [24]: 359) }\end{array}$ & & $\mathrm{X}$ & & & & & \\
\hline & Fasp3_16Bu-2 & $\begin{array}{l}\text { Facelina sp. (Facelina sp. } 4 \text { in } \\
\text { Gosliner et al. [24]: 359) }\end{array}$ & & $x$ & & & & & \\
\hline & Fasp8_16Bu-1 & $\begin{array}{l}\text { Facelina sp. (Facelina sp. } 8 \\
\text { in Gosliner et al. [24]: 360) }\end{array}$ & & $x$ & & & & & \\
\hline & & Favorinus japonicus Baba, 1949 & $x$ & $x$ & & & & & \\
\hline & & Favorinus mirabilis Baba, 1955 & & $x$ & & & & & \\
\hline & & $\begin{array}{c}\text { Favorinus tsuruganus Baba and } \\
\text { Abe, } 1964\end{array}$ & $x$ & & & & & & \\
\hline & Fasp1-18Ba-1-2 & $\begin{array}{c}\text { Favorinus sp. } 1 \\
\text { (in Gosliner et al. [24]: 363) }\end{array}$ & $x$ & & & & & & \\
\hline & Mojo18Ba-1 & Moridilla sp. a & $x$ & & & & & & \\
\hline & & $\begin{array}{c}\text { Noumeaella sp. } 2 \\
\text { (in Gosliner et al. [24]: 367) }\end{array}$ & $x$ & & & & & & \\
\hline & & $\begin{array}{c}\text { Noumeaella sp. } 3 \\
\text { (in Gosliner et al. [24]: 367) }\end{array}$ & $x$ & $x$ & & & & & \\
\hline
\end{tabular}


Table 3. Cont

\begin{tabular}{|c|c|c|c|c|c|c|c|c|c|}
\hline \multicolumn{10}{|c|}{ Expedition in Bangka Archipelago and Adjacent Islands } \\
\hline Higher Taxon Affiliation & Identifier & Species Name & $\begin{array}{c}\text { Exp } \\
\text { Bangka } \\
\text { 2017-2018 }\end{array}$ & $\begin{array}{c}\text { Exp Bunaken } \\
2015-2017\end{array}$ & $\begin{array}{l}\text { Exp } \\
\text { Bunaken } \\
2003\end{array}$ & $\begin{array}{c}\text { Exp } \\
\text { Sangihe } \\
2016\end{array}$ & $\begin{array}{c}\text { Exp } \\
\text { Lembeh } \\
2018\end{array}$ & $\begin{array}{l}\text { Lembeh } \\
\text { Sea Slug } \\
\text { Forum } \\
2001-2010\end{array}$ & $\begin{array}{c}\text { Tonozuka } \\
2003\end{array}$ \\
\hline & Nosp6_17Bu-1-2 & $\begin{array}{l}\text { Noumeaella sp. (Noumeaella cf. sp. } \\
6 \text { in } \\
\text { Gosliner et al. [24]: 368) }\end{array}$ & & $\mathrm{x}$ & & & & & \\
\hline & Nosp12_16Bu-1 & $\begin{array}{l}\text { Noumeaella sp. (Noumeaella sp. } 12 \\
\text { in Gosliner et al. [24]: 367) }\end{array}$ & & $x$ & & & & & \\
\hline & & $\begin{array}{c}\text { Noumeaella sp. 13 } \\
\text { (in Gosliner et al. [24]: 369) }\end{array}$ & $\mathrm{x}$ & & & & & & \\
\hline & $\begin{array}{l}\text { Nosp2_15Bu 1 } \\
\text { Nosp2_16Bu-1-6 }\end{array}$ & Noumeaella sp. & & $x$ & & & & & \\
\hline & & $\begin{array}{l}\text { Phyllodesmium briareum (Bergh, } \\
\text { 1896) }\end{array}$ & $\mathrm{X}$ & $\mathrm{X}$ & $\mathrm{x}$ & & & $\mathrm{x}$ & \\
\hline & & $\begin{array}{l}\text { Phyllodesmium cf. crypticum } \\
\text { Rudman, } 1981\end{array}$ & $x$ & & & & & & \\
\hline & & $\begin{array}{l}\text { Phyllodesmium jakobsenae } \\
\text { Burghardt and Wägele, } 2004\end{array}$ & & & $\mathrm{x}$ & & & & \\
\hline & & $\begin{array}{l}\text { Phyllodesmium lizardense Burghardt, } \\
\text { Schrödl and Wägele, } 2008\end{array}$ & $x$ & & & & & & \\
\hline & & $\begin{array}{c}\text { Phyllodesmium longicirrum (Bergh, } \\
\text { 1905) }\end{array}$ & $\mathrm{x}$ & & & & & & \\
\hline & & $\begin{array}{l}\text { Phyllodesmium magnum } \\
\text { Rudman, } 1991\end{array}$ & $x$ & & & & & $x$ & \\
\hline & & $\begin{array}{l}\text { Phyllodesmium parangatum Ortiz } \\
\text { and Gosliner, } 2003\end{array}$ & $x$ & & & & & & \\
\hline & & $\begin{array}{c}\text { Phyllodesmium pecten Rudman, } \\
1981\end{array}$ & $x$ & & & & & & \\
\hline
\end{tabular}


Table 3. Cont

\begin{tabular}{|c|c|c|c|c|c|c|c|c|c|}
\hline \multicolumn{10}{|c|}{ Expedition in Bangka Archipelago and Adjacent Islands } \\
\hline Higher Taxon Affiliation & Identifier & Species Name & $\begin{array}{c}\text { Exp } \\
\text { Bangka } \\
\text { 2017-2018 }\end{array}$ & $\begin{array}{l}\text { Exp Bunaken } \\
\text { 2015-2017 }\end{array}$ & $\begin{array}{c}\text { Exp } \\
\text { Bunaken } \\
2003\end{array}$ & $\begin{array}{l}\text { Exp } \\
\text { Sangihe } \\
2016\end{array}$ & $\begin{array}{c}\text { Exp } \\
\text { Lembeh } \\
2018\end{array}$ & $\begin{array}{c}\text { Lembeh } \\
\text { Sea Slug } \\
\text { Forum } \\
\text { 2001-2010 }\end{array}$ & $\begin{array}{c}\text { Tonozuka } \\
2003\end{array}$ \\
\hline & & $\begin{array}{c}\text { Phyllodesmium poindimiei (Risbec, } \\
\text { 1928) }\end{array}$ & $\mathrm{x}$ & $\mathrm{x}$ & & & & & \\
\hline & & $\begin{array}{c}\text { Phyllodesmium rudmani } \\
\text { Burghardt and Gosliner, } 2006\end{array}$ & & & $\mathrm{X}$ as sp 1 & & & $\mathrm{X}$ as sp. 11 & \\
\hline & & $\begin{array}{l}\text { Phyllodesmium undulatum Moore } \\
\text { and Gosliner, } 2014\end{array}$ & & & & & & & $\mathrm{x}$ \\
\hline & & $\begin{array}{l}\text { Phyllodesmium sp. } 9 \text { (Sea Slug } \\
\text { Forum) }\end{array}$ & & & & & & $\mathrm{x}$ & \\
\hline & & $\begin{array}{l}\text { Pteraeolidia semperi (Bergh, 1870) as } \\
\text { P. ianthina (Burghardt and Sea Slug } \\
\text { Forum) }\end{array}$ & $\mathrm{x}$ & $\mathrm{x}$ & $\mathrm{x}$ & & & $\mathrm{x}$ & $\mathrm{x}$ \\
\hline & Fasp17Bu-1 & Facelinidae sp. & & $x$ & & & & & \\
\hline & Fasp17Bu-1 & Facelinidae sp. & & $x$ & & & & & \\
\hline \multicolumn{10}{|c|}{ Eupulmonata } \\
\hline Onchidiidae & & Peronia sp. & $\mathrm{x}$ & & & & & & \\
\hline Total number & & & 149 & 172 & 75 & 23 & 27 & 85 & 25 \\
\hline
\end{tabular}


Only 10 species (Thuridilla gracilis, Chromodoris annae, C. strigata, Glossodoris cincta, Goniobranchus geometricus, G. reticulatus, Hypselodoris tryoni, Phyllidia ocellata, P. varicosa, and Phyllidiella pustulosa) are recorded from all areas. Of these, C. annae, P. varicosa, and P. pustulosa show the broadest distribution with records from nearly all dive sites of the four study areas, and can probably be considered as the most dominant species in North Sulawesi. However, there are also distinct differences in species composition at each of the various study areas. A few of them we would like to address here, with an emphasis on Bunaken National Park (BNP) and Lembeh Strait (LS). A much higher number of cephalaspideans and sacoglossans are now recorded from BNP than from BA or LS. However, the sacoglossan Thuridilla gracilis is very common in BA and LS, but very rare in BNP. Future studies will show which of the recently identified $14 \mathrm{~T}$. gracilis lineages [31] are present. In contrast, its congener $T$. lineolata is a common species in BNP, especially in the lagoons behind the fringing reefs. This habitat structure is less common in BA and LS and is probably the reason why T. lineolata was not found in BA (but recorded in LS).

Six species of Nembrotha were collected in BA, but only two of these species, the highly conspicuous Nembrotha kubaryana and N. cristata, are reported from BNP, which are also very common in BA. Members of the genus feed on ascidians, which usually need higher nutrition in the water column [43]. No bryozoan-feeding members of the genus Polycera are recorded from BA, although several species are recorded from BNP [16]. However, the bryozoan-feeding genus Tambja is represented by two species in BA, but only one in BNP.

Halgerda batangas was represented in BA with 10 specimens from five different localities, a much higher number than the records in BNP [16]. Localities in the two different study areas are similar in as much as all sites with Halgerda present were noted to have a highly diverse sponge community. Why diversity and also number of specimens of Halgerda species seems to be higher in BA and LS is therefore not clear, but can probably be explained by the distribution and environmental needs of the food sponges [24,44].

Within Chromodorididae, the genus Hypselodoris is quite common in BA and LS, with 11 and 10 species, respectively. In BNP, five species are recorded: the three species $H$. apolegma, $H$. maculosa, and H. tryoni are also distributed in BA and LS, but two undescribed species are only recorded from BNP.

Some taxa are small and very inconspicuous, and therefore seldom reported by citizen scientists. The genus Dermatobranchus has many small forms with cryptic coloration and is therefore easily overlooked. Our study revealed six different species in BA; Eisenbarth et al. [16] also recorded six species from BNP; however, the overlap of species includes only one undescribed species of Dermatobranchus. None of these Dermatobranchus species are recorded from LS [21,39].

Dotidae are represented by Doto and Kabeiro in North Sulawesi. Interestingly, the genus Kabeiro was quite common in BNP, but was not found in BA. Members of this genus exclusively feed on hydroids of the family Plumulariidae, which are less common in BA.

Many biotic and abiotic factors influence both the occurrence and distribution of sea slugs. The main island of Bunaken National Park, Bunaken Island, is formed by an old reef and is characterized by fringing reefs with wall-like structures, exposed to hydrodynamics varying in strength (e.g., high waves and strong currents). Lagoons behind the fringing reefs are formed by white sand partly covered by seagrass or mangroves and are influenced by the tides. Lembeh Strait, located in the south-east of the Bangka Archipelago, is of volcanic origin and dominated by slopes with coral patches, volcanic sand slopes, and beds. Fringing reefs, typical for Bunaken National Park, are uncommon in LS and BA. Bangka Island lies between these two areas and supports both habitats: it is partly fringed by reefs, and areas are partly dominated by volcanic sands. However, habitat structure and substrate are not the only factors that influence species composition; temperature and water currents also have a strong effect on the connectivity of populations within these three localities, affecting the distribution of both heterobranch predator and prey larvae [45-47]. Understanding how these factors contribute to the differences would require much more information about the lifestyle of those particular species that are more common or rare in the respective geographic areas. 


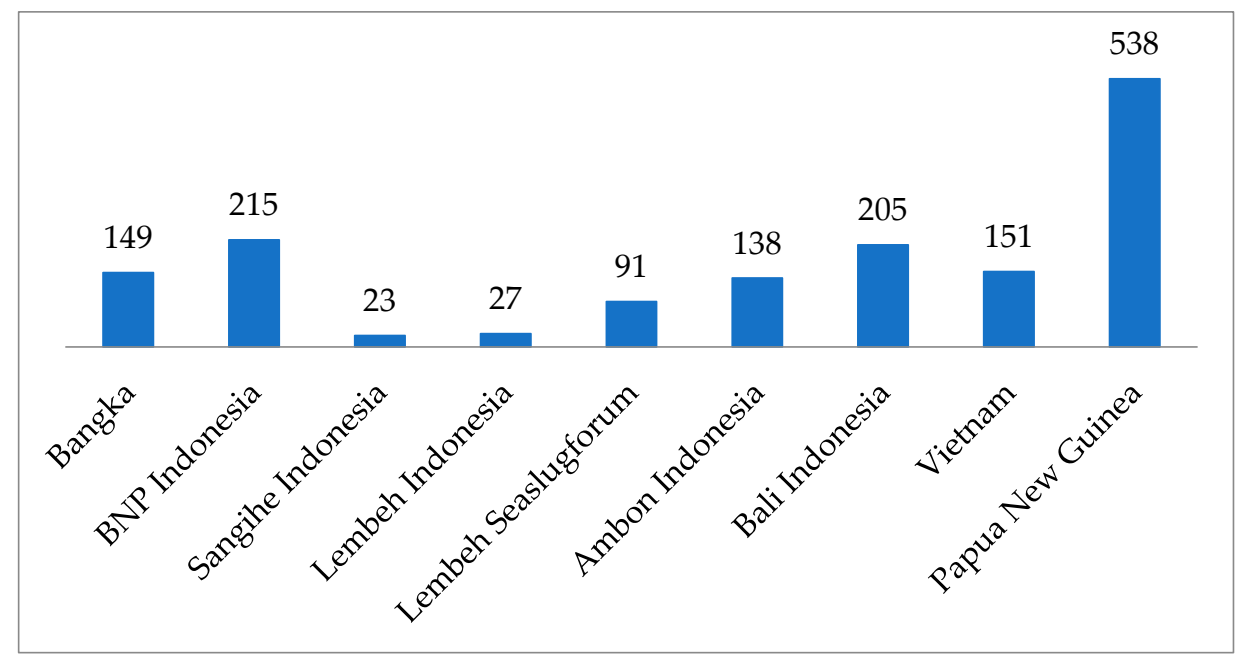

Figure 28. Species diversity from Bangka Archipelago compared with other studies in Indonesia and areas nearby. Data taken from the following literature: BNP, Indonesia [15,16]; Sangihe, Indonesia [18]; Lembeh Island, Indonesia [17,21]; Ambon, Indonesia [3-6]; Bali, Indonesia [39]; Vietnam [22]; and Papua New Guinea [48].

\section{Conclusions}

When comparing our study in BA with other available studies from Indonesia, e.g., Ambon, Bali, and Papua New Guinea [2,16,38,48], we can consider the number of 149 species as reasonable for this region after only a few collection events. Only the study in Papua New Guinea exhibits a much higher number of species (Figure 28), probably due to the exceptionally high collection effort in this area [48]. We also cannot exclude the influence of seasons on the success of collection. Larkin et al. [49] showed a considerable difference in the abundance of marine Heterobranchia seen during the day and night, a factor that we took into consideration to a certain extent. However, we collected only during the late dry season, another factor that may influence overall diversity numbers $[49,50]$.

The comparison between the various regions in North Sulawesi (Figure 27) and other Indonesian regions (Figure 28) allows the conclusion that the true number of species is actually certainly much higher than those recorded in these studies, not only in Bangka Archipelago but also in the other Indonesian areas sampled to date. A continuous increase in species records in the Indo-Pacific can already be seen when comparing the identification book of Gosliner et al. published in 2015 [24] with his follow-up study published in 2018 [51], with an increase of more than 100 species, from less than 2000 documented ones in the Indo-Pacific to more than 2100. Although we have mainly used the version from 2015, we subsequently checked our findings with the latest version, which did not reveal new information or species relevant for our study. Thus, our results contribute to the overall species numbers for the Indo-Pacific, and they form a baseline for future monitoring in the region, which still appears to be unaffected by environmental stress factors. According to the planktonic community index based on recent planktonic studies, the marine environment of BA is considered to be in a good condition [52]. However, Ponti et al. [19] consider the health of the corals in the area as critical, despite the low impact of disease, and refer to the general problems in BA of increased human activities, including mining, which irredeemably compromises reef health [19]. Snorkeling in front of the destroyed area at the mining site did not result in any records of nudibranchs, due to the recent stone blocks dumped in front of the site, where only a few corals and sponges were observed to colonize them. Probably due to land erosion and the lack of mangroves to filter nutrients and sediments, the water was extremely murky, and the high concentration of tiny jellyfish might be an additional indicator of eutrophication [50,53]. However, the nearby investigated areas, such as Sipi, revealed a pristine environment, and 13 heterobranch species were recorded from this locality (Table 2) when diving for approximately one hour with five divers involved. Since the mining project stopped 
recently, the highly disturbed habitat in front of the mines will provide a good study area for future studies of recolonization and recovery, and the results of this study with detailed information from each locality are a good baseline for comparison.

Author Contributions: Conceptualization: H.W. Data curation: A.P., N.U., N.A.M., M.R.S., I.G.D., R.R.K., M.P. and H.W.; Formal analysis: A.P., H.W., N.U. and N.Y.; Funding acquisition: H.W.; Investigation: A.P., N.U. and H.W.; Project administration: H.W.; Resources: M.R.S. and H.W.; Supervision: H.W.; Validation: H.W. and N.Y.; Visualization: A.P.; Writing—original draft: A.P. and H.W.; Writing—review and editing: N.Y. and H.W. All authors have read and agreed to the published version of the manuscript.

Funding: This research was funded by the Federal Ministry of Education and Research (BMBF) in the framework of the "Biodiversity and Health—from Biodiversity to Biomedical Innovation" program to Heike Wägele (IndoBio project, grant 16GW0118). Nani Undap and Adelfia Papu have grants provided by the German Academic Exchange System (DAAD).

Acknowledgments: We are very grateful to Gianni Valenti (Siena, Italy), who shared his pictures with us for this study. We thank Wolfgang Wägele (Bonn, Germany) for assistance in collecting several species. The material is property of the Collection of the Sam Ratulangi University (Faculty of Fisheries and Marine Science) (SRU2017/01, SRU2018/02) and loaned to the corresponding author for further investigation. We thank Claudia Etzbauer who helped with molecular work. We also thank the staff from Coral Eye on Bangka Island for their continuous support. We also would like to thank three anonymous reviewers for their valuable comments and help to improve our manuscript. We are very grateful to Markus Lasut and Grevo Gerung (Faculty of Fishery and Marine Sciences, Manado) for continuing support and help with the ABS paperwork to follow the Nagoya Protocol. Finally, we are grateful to the Republic of Indonesia for making this research possible. Copyright of figures: Zoological Research Museum Alexander Koenig.

Conflicts of Interest: The authors declare no conflicts of interest.

\section{References}

1. Kaligis, F.; Eisenbarth, J.H.; Schillo, D.; Dialao, J.; Schäberle, T.F.; Böhringer, N.; Bara, R.; Reumschüssel, S.; König, G.M.; Wägele, H. Second survey of heterobranch sea slugs (Mollusca, Gastropoda, Heterobranchia) from Bunaken National Park, North Sulawesi, Indonesia-How much do we know after 12 years. Mar. Biodiver. Rec. 2018, 11, 1-20. [CrossRef]

2. Naturalis Biodiversity Center, Repository, Rumphius Biohistorical. Available online: http: //www.repository.naturalis.nl/cgi/b/bib/bib-idx?type=simple;c=naturalis;rgn1=entire\%20record;q1= Rumphius\%20Biohistorical;Submit.x=14;Submit.y=11;lang=en;sort=year;cc=naturalis;view=reslist;fmt= short;page $=$ reslist; size $=10 ;$ start $=1 ;$ sid $=($ accessed on 9 February 2019).

3. Yonow, N. Results from the Rumphius Biohistorical Expedition to Ambon (1990). Part 16. Nudibranchia-Dendronotina, Arminina, Aeolidina, and Doridina (Mollusca: Gastropoda: Heterobranchia). Arch. Molluskenkd. 2017, 146, 135-172. [CrossRef]

4. Yonow, N. Results from the Rumphius Biohistorical Expedition to Ambon (1990). Part 15. The suborder Doridina (Mollusca, Gastropoda, Opisthobranchia, Nudibranchia). Zool. Meded. 2011, 85, 905-956.

5. Yonow, N. Results from the Rumphius Biohistorical Expedition to Ambon (1990). Part 11. Doridacea of the families Chromodorididae and Hexabranchidae (Mollusca, Gastropoda, Opisthobranchia, Nudibranchia). Zool. Meded. 2001, 75, 1-50.

6. Yonow, N.; Jensen, K.R. Results of the Rumphius Biohistorical Expedition to Ambon (1990). Part 17. The Cephalaspidea, Anaspidea, Pleurobranchida, and Sacoglossa (Mollusca: Gastropoda: Heterobranchia). Arch. Molluskenkd. 2018, 147, 1-48. [CrossRef]

7. Böhringer, N.; Fisch, K.M.; Schillo, D.; Bara, R.; Hertzer, C.; Grein, F.; Eisenbarth, J.-H.; Kaligis, F.; Schneider, T.; Wägele, H.; et al. Antimicrobial potential of bacteria associated with marine sea slugs from North Sulawesi, Indonesia. Front. Microbiol. 2017, 8, 1092. [CrossRef]

8. Burghardt, I.; Gosliner, T.M. Phyllodesmium rudmani (Mollusca: Nudibranchia: Aeolidoidea), a new solar powered species from the Indo-West Pacific with data on its symbiosis with zooxanthellae. Zootaxa 2008, 1308, 31-47. [CrossRef]

9. Okino, T.; Yoshimura, E.; Hirota, H.; Fusetani, N. New antifouling sesquiterpenes from four nudibranchs of the family Phyllidiidae. Tetrahedron 1996, 52, 9447-9454. [CrossRef] 
10. Fisch, K.M.; Hertzer, C.; Böhringer, N.; Wuisan, Z.G.; Schillo, D.; Bara, R.; Kaligis, F.; Wägele, H.; König, G.M.; Schäberle, T.F. The potential of Indonesian gastropods found around Bunaken Island for production of bioactive compounds. Mar. Drugs 2017, 15, 384. [CrossRef]

11. Haber, M.; Cerfeda, S.; Carbone, M.; Calado, G.; Gaspar, H.; Neves, R.; Maharajan, V.; Cimino, G.; Gavagnin, M.; Ghiselin, M.T.; et al. Coloration and defense in the nudibranch gastropod Hypselodoris fontandraui. Biol. Bull. 2010, 218, 181-188. [CrossRef]

12. Wells, F.E.; Bryce, C.W. Sea Slugs and Their Relatives of Western Australia; Western Australian Museum: Perth, Australia, 1993; p. 184.

13. Nimbs, M.J.; Larkin, M.; Davis, T.R.; Harasti, D.; Willan, R.C.; Smith, S.D.A. Southern range extensions for twelve heterobranch sea slugs (Gastropoda: Heterobranchia) on the eastern coast of Australia. Mar. Biodivers. Rec. 2016, 9, 1-12. [CrossRef]

14. Nimbs, M.J.; Smith, S.D.A. Beyond Captricornia: Tropical sea slugs (Gastropoda, Heterobranchia) extend their distributions into the Tasman Sea. Diversity 2018, 10, 99. [CrossRef]

15. Burghardt, I.; Carvalho, R.; Eheberg, D.; Gerung, G.; Kaligis, F.; Mamangkey, G.; Schrödl, M.; Schwabe, E.; Vonnemann, V.; Wägele, H. Molluscan diversity at Bunaken National Park, Sulawesi. J. Zool. Soc. Wallacea 2006, 2, 29-43.

16. Eisenbarth, J.-H.; Undap, N.; Papu, A.; Schillo, D.; Dialao, J.; Reumschüssel, S.; Kaligis, F.; Bara, R.; Schäberle, T.F.; König, G.M.; et al. Marine Heterobranchia (Gastropoda, Mollusca) in Bunaken National Park, North Sulawesi, Indonesia-A follow-up diversity study. Diversity 2018, 10, 127. [CrossRef]

17. Ompi, M.; Lumoindong, F.; Undap, N.; Papu, A.; Wägele, H. Monitoring marine Heterobranchia in Lembeh Strait, North Sulawesi (Indonesia), in a changing environment. AACL Bioflux 2019, 12, 664-667.

18. Undap, N.; Papu, A.; Schillo, D.; Ijong, F.G.; Kaligis, F.; Lepar, M.; Hertzer, C.; Böhringer, N.; König, G.M.; Schäberle, T.F.; et al. First survey of heterobranch sea slugs (Mollusca, Gastropoda) from the island Sangihe, North Sulawesi, Indonesia. Diversity 2019, 11, 1-30. [CrossRef]

19. Ponti, M.; Fratangeli, F.; Dondi, N.; Reinach, M.S.; Serra, C.; Sweet, M.J. Baseline reef health surveys at Bangka Island (North Sulawesi, Indonesia) reveal new threats. PeerJ 2016, 4, e2614. [CrossRef]

20. Layton, K.K.S.; Gosliner, T.M.; Wilson, N.G. Flexible color check patterns obscure identification and mimicry in Indo-Pacific Chromodoris nudibranchs (Gastropoda: Chromodorididae). Mol. Phyl. Evol. 2018, 124, 27-36. [CrossRef]

21. Sea Slug Forum Home Page. Available online: http://www.seaslugforum.net/ (accessed on 10 December 2019).

22. Martynov, A.V.; Korshunova, T.A. Opisthobranch molluscs of Vietnam (Gastropoda: Opisthobranchia). In Benthic Fauna of the Bay of Nhatrang, Southern Vietnam 2; Britayev, T.Z., Pavlov, D.S., Eds.; KMK Scientific Press: Moscow, Russia, 2012; pp. 142-257.

23. Burghardt, I.; Wägele, H. The symbiosis between the 'solar-powered' nudibranch Melibe engeli Risbec, 1937 (Dendronotoidea) and Symbiodinium sp. (Dinophyceae). J. Moll. Stud. 2014, 80, 508-517. [CrossRef]

24. Gosliner, T.M.; Valdés, A.; Behrens, D.W. Nudibranch \& Sea Slug Identification Indo-Pacific; New World Publications: Jacksonville, FL, USA, 2015; p. 408.

25. Stoffels, B.E.M.W.; van der Meij, S.E.T.; Hoeksema, B.W.; van Alphen, J.; van Alen, T.; Meyers-Munoz, M.A.; de Voogd, N.J.; Tuti, Y.; van der Velde, G. Phylogenetic relationships within the Phyllidiidae (Opisthobranchia, Nudibanchia). ZooKeys 2016, 605, 1-35. [CrossRef]

26. World Register of Marine Species. Available online: http://www.marinespecies.org/ (accessed on 17 January 2020).

27. Epstein, H.E.; Hallas, J.M.; Johnson, R.F.; Lopez, A.; Gosliner, T.M. Reading between the lines: Revealing cryptic species diversity and color patterns in Hypselodoris nudibranchs (Mollusca: Heterobranchia: Chromodorididae). Zool. J. Linn. Soc. 2019, 186, 116-189. [CrossRef]

28. Diepenbroek, M.; Glökner, F.O.; Grobe, P.; Güntsch, A.; Huber, R.; König-Ries, B.; Kostadinov, I.; Nieschulze, J.; Seeger, B.; Tolksdorf, R.; et al. Towards an integrated biodiversity and ecological research data management and archiving platform: The German Federation for the Curation of Biological Data (GFBio). In Proceedings of the GI, Informatik 2014—Big Data Komplexität meistern, Stuttgart, Germany, January 2014; Plödereder, E., Grunske, L., Schneider, E., Ull, D., Eds.; Köllen Verlag: Bonn, Germany, 2014; pp. 1711-1724. 
29. Golestani, H.; Crocetta, F.; Padula, V.; Camacho, Y.; Langeneck, J.; Poursanidis, D.; Pola, M.; Yokes, M.B.; Cervera, J.L.; Jung, D.W.; et al. The little Aplysia coming of age: From one species to a complex of species complexes in Aplysia parvula (Mollusca: Gastropoda: Heterobranchia). Zool. J. Linn. Soc. 2019, 187, 279-330. [CrossRef]

30. Bouchet, P.; Rockroi, J.-P.; Hausdorf, B.; Kaim, A.; Kano, Y.; Nützel, A.; Parkvaev, P.; Schrödl, M.; Strong, E.E. Revised classification, nomenclator and typification of gastropod and monoplacophoran families. Malacologia 2017, 61, 1-526. [CrossRef]

31. Martín-Hervás, M.R.; Carmona, L.; Malaquias, M.A.E.; Krug, P.J.; Gosliner, T.; Cervera, J.L. Integrative Systematics of the Genus Thuridilla Bergh, 1872 (Mollusca, Gastropoda, Heterobranchia) reveals a cryptic radiation of polymorphic sea slug species. In Proceedings of the World Congress of Malacology, Pacific Grove, CA, USA, 11-16 August 2019; California Academy of Sciences: San Francisco, CA, USA; p. 119. Available online: https://www.calacademy.org/sites/default/files/final_abstract_volume_2019_1.pdf (accessed on 13 January 2020).

32. Matsuda, S.B.; Gosliner, T.M. Glossing over cryptic species: Descriptions of four new species of Glossodoris and three new species of Doriprismatica (Nudibranchia: Chromodorididae). Zootaxa 2018, 4444, 501-529. [CrossRef] [PubMed]

33. Winters, A.E.; Wilson, N.G.; van den Berg, C.P.; How, M.J.; Endler, J.A.; Marshall, N.J.; White, A.M.; Garson, M.J.; Cheney, K.L. Toxicity and taste: Unequal chemical defences in a mimicry ring. Proc. R. Soc. B Biol. Sci. 2018, 285. [CrossRef] [PubMed]

34. Coleman, N.; Cobb, G.; Mullins, D. Nudibranchs Encyclopedia, Catalogue of Asia/Indo Pacific Sea Slugs, 2nd ed.; Masalai Press: Oakland, CA, USA, 2015; p. 312. ISBN 978-0-9714127-9-8.

35. Korshunova, T.; Martynov, A.; Picton, B. Ontogeny as an important part of integrative taxonomy in tergipedid aeolidaceans (Gastropoda: Nudibranchia) with a description of a new genus and species from the Barents Sea. Zootaxa 2017, 4324, 1-22. [CrossRef]

36. Schillo, D.; Wipfler, B.; Undap, N.; Böhringer, N.; Eisenbarth, J.-H.; Kaligis, F.; Bara, R.; Schäberle, T.F.; König, G.M.; Wägele, H. Description of a new Moridilla species from North Sulawesi, Indonesia (Mollusca: Nudibranchia: Aeolidioidea)—based on MicroCT, histological and molecular analyses. Zootaxa 2019, 4652, 265-295. [CrossRef]

37. Yasman. Observation on the feeding of nudibranch Phyllidia varicosa Lamarck, 1801 on the sponge Axinyssa cf. aculeata Wilson, 1925 in coral reefs of Pramuka Island, Thousands Islands National Park, Indonesia. Makara Sains 2003, 7, 15-21.

38. Alqudah, A.A.; Shahbudin, S.; Deny, S.; Hadry, N.F.; Khodzori, M.F.A.; Yusof, M.H.; Rani, M.H. Observations on nudibranch behaviour patterns under laboratory conditions. J. Teknol. 2016, 78, 167-171. [CrossRef]

39. Tonozuka, T. Opisthobranchs of Bali and Indonesia; Hankyu Communications Co. Ltd.: Tokyo, Japan, 2003; p. 164.

40. Pasternak, G.; Galil, B.S. An established population of the alien sea slug Elysia grandifolia Kelaart, 1858 (Mollusca, Opisthobranchia, Elysiidae) off the Mediterranean coast of Israel. BioInvasions Rec. 2012, 1, 221-223. [CrossRef]

41. Padula, V.; Bahia, J.; Stöger, I.; Camacho-Garcia, Y.; Malaquias, M.A.E.; Cervera, J.L.; Schrödl, M. A test of color-based taxonomy in nudibranchs: Molecular phylogeny and species delimitation of the Felimida clenchi (Mollusca: Chromodorididae) species complex. Mol. Phyl. Evol. 2016, 103, 215-229. [CrossRef] [PubMed]

42. Smith, S.D.A.; Davis, T.R. Slugging it out for science: Volunteers provide valuable data on the diversity and distribution of heterobranch sea slugs. Moll. Res. 2019, 39, 214-233. [CrossRef]

43. Pola, M.; Cervera, J.L.; Gosliner, T.M. Revision of the Indo-Pacific genus Nembrotha (Nudibranchia: Dorididae: Polyceridae), with a description of two new species. Sci. Mar. 2008, 72, 145-183. [CrossRef]

44. Tibiriçá, Y.; Pola, M.; Cervera, J.L. Astonishing diversity revealed: An annotated and illustrated inventory of Nudipleura (Gastropoda: Heterobranchia) from Mozambique. Zootaxa 2017, 4359, 1-133. [CrossRef]

45. Fetzer, I. Reproduction strategies and distribution of larvae and juveniles of benthic soft-bottom invertebrates in the Kara Sea (Russian Arctic). The influence of river discharge on the structure of benthic communities: A larval approach. Ph.D. Thesis, Bremen University, Bremen, Germany, 2004. Available online: http: //hdl.handle.net/10013/epic.21857.d001 (accessed on 28 January 2020).

46. García, F.J.; Bertsch, H. Diversity and distribution of the Gastropoda Opisthobranchia from the Atlantic Ocean: A global biogeographic approach. Sci. Mar. 2009, 73, 153-160. [CrossRef] 
47. Brodie, G.D.; Brodie, J.E. Species diversity and habitat selection in opisthobranch gastropods on two adjacent reefs in Fiji. South Pacific, J. Nat. Sci. 1995, 14, 97-113.

48. Gosliner, T.M. Biodiversity of tropical opisthobranch gastropod faunas. In Proceedings of the Seventh International Coral Reef Symposium, Guam, Micronesia, 22-27 June 1992; pp. 702-709.

49. Larkin, M.F.; Smith, S.D.A.; Willan, R.C.; Davis, T.R. Diel and seasonal variation in heterobranch sea slug assemblages within an embayment in temperate eastern Australia. Mar. Biodiv. 2018, 48, 1541-1550. [CrossRef]

50. Smith, S.D.A.; Nimbs, M.J. Quantifying temporal variation in heterobranch (Mollusca: Gastropoda) sea slug assemblages: Tests of alternate models. Moll. Res. 2017, 37, 140-147. [CrossRef]

51. Gosliner, T.M.; Valdés, A.; Behrens, D.W. Nudibranch \& Sea Slug Identification Indo-Pacific; New World Publications: Jacksonville, FL, USA, 2018; p. 451.

52. Usman, M.S.; Kusen, J.D.; Rimper, J.R.T.S.L. Struktur Komunitas Plankton di Perairan Pulau Bangka Kabupaten Minahasa Utara. J. Pesisir dan Laut Trop. 2013, 2, 51-57. [CrossRef]

53. Qu, C.F.; Song, J.M.; Li, N. Causes of jellyfish blooms and their influence on marine environment. Ying Yong Sheng Tai Xue Bao. 2014, 25, 3701-3712.

(C) 2020 by the authors. Licensee MDPI, Basel, Switzerland. This article is an open access article distributed under the terms and conditions of the Creative Commons Attribution (CC BY) license (http://creativecommons.org/licenses/by/4.0/). 